\title{
Characterisation of star-planet systems
}

\author{
Dissertation \\ zur Erlangung des mathematisch-naturwissenschaftlichen \\ Doktorgrades \\ "Doctor rerum naturalium" \\ der Georg-August-Universität Göttingen \\ im Promotionsprogramm PROPHYS \\ der Georg-August University School of Science (GAUSS)
}

vorgelegt von

\section{Vera Maria Passegger}

aus Graz, Österreich

Göttingen, 2017 
$\underline{\text { Betreuungsausschuss }}$

Prof. Dr. Ansgar Reiners, Stellare Astrophysik, Institut für Astrophysik Göttingen

Dr. Sandra Jeffers, Stellare Astrophysik, Institut für Astrophysik Göttingen

Mitglieder der Prüfungskommission

Referent: Prof. Dr. Ansgar Reiners

Institut für Astrophysik, Georg-August-Universität, Göttingen, Deutschland

Korreferent: Prof. Dr. Artie P. Hatzes

Thüringer Landessternwarte Tautenburg, Physikalisch-Astronomische Fakultät, Friedrich-Schiller-Universität, Jena, Deutschland

Weitere Mitglieder der Prüfungskommission:

Prof. Dr. Wolfram Kollatschny

Institut für Astrophysik, Georg-August-Universität, Göttingen, Deutschland

Prof. Dr. Stefan Dreizler

Institut für Astrophysik, Georg-August-Universität, Göttingen, Deutschland

Prof. Dr. Stefan Mathias

I. Physikalisches Institut, Georg-August-Universität, Göttingen, Deutschland

Prof. Dr. Claus Ropers

IV. Physikalisches Instiut, Georg-August-Universität, Göttingen, Deutschland

Tag der mündlichen Prüfung: 27. April 2017 


\section{Abstract}

After the first confirmed detection of an exoplanet in 1995, their number has significantly increased to 3557 confirmed planets around other stars 1 . The most successful techniques for planet search are transit and radial-velocity observations. Due to the large mass ratio between the star and the planet, $\mathrm{M}$ dwarfs are favourable for radial-velocity surveys. The CARMENES instrument mounted at the $3.5 \mathrm{~m}$ telescope at Calar Alto Observatory in Spain consists of two high-precision spectrographs to search for Earth-sized planets in the habitable zones of $\mathrm{M}$ dwarfs. The high-resolution $(R \sim 82000)$ spectrographs operate in the visible $(0.55-0.95 \mu \mathrm{m})$ and near-infrared $(0.95-1.7 \mu \mathrm{m})$ wavelength range with precisions of around $1 \mathrm{~ms}^{-1}$. In order to characterise a potential planet it is necessary to characterise the host star. For CARMENES science preparation around 1700 spectra of 523 stars have been taken with other high-resolution spectrographs like CAFE, FEROS and HRS, to analyse the target candidate sample. I developed an algorithm to determine fundamental parameters for these stars, i.e. effective temperature $\left(T_{\text {eff }}\right)$, surface gravity $(\log g)$ and metallicity $[\mathrm{Fe} / \mathrm{H}]$. The determination of stellar parameters in low-mass $\mathrm{M}$ stars is more difficult than for hotter stars like the Sun, since their lower temperatures lead to the formation of molecules showing dense forests of lines in the stellar spectra. Instead of a line-by-line approach, a more complex full spectral synthesis is necessary. The PHOENIX-ACES models are up-to-date synthetic spectra especially designed for low-temperature stellar atmospheres. The algorithm fits the PHOENIX-ACES models to the observed spectra and determines the best fit using a downhill-simplex method and $\chi^{2}$-minimisation. Spectral lines and ranges are used, which are especially sensitive to $T_{\text {eff }}, \log g$ and $[\mathrm{Fe} / \mathrm{H}]$. My method gives accurate parameters for a large sample of 323 $\mathrm{M}$ dwarfs with uncertainties of $93 \mathrm{~K}$ for $T_{\text {eff }}, 0.29$ dex for $\log g$, and 0.25 dex for metallicity. Comparisons with literature values present excellent agreement in temperature and surface gravity. However, in metallicity there is a larger spread compared to measurements from literature. The overall sample statistically agrees with other samples analysed in earlier studies, although for single stars the deviations can be more than $1 \sigma$. Accurate metallicity determinations in low-mass stars are very challenging and possible explanations for metallicity discrepancies, e.g. inconsistencies in synthetic models or the use of different determination methods, definitely need further and more detailed investigations.

\footnotetext{
${ }^{1}$ www.exoplanet.eu, Dec. 2016
} 



\section{Contents}

1. Introduction 1

1.1. Exoplanets . . . . . . . . . . . . . . . . . . . . . . . 1

1.1.1. Habitable zone ................... . . . 2

1.1.2. Radial-velocity method . . . . . . . . . . . . . . . . 4 4

1.1.3. Transit method . . . . . . . . . . . . . . . . 5

1.1.4. Direct Imaging . . . . . . . . . . . . . . . . . . . . . . . 6

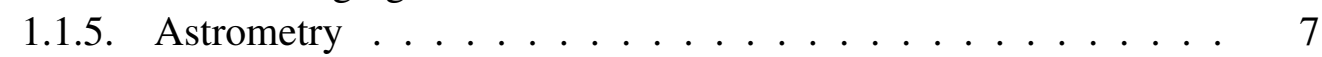

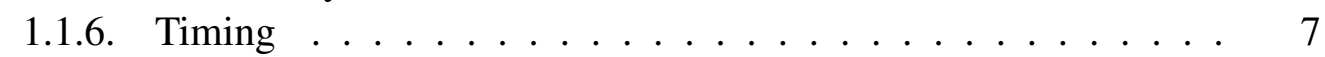

1.1.7. Microlensing . . . . . . . . . . . . . . . . . . . . . . 8

1.2. Spectroscopy . . . . . . . . . . . . . . . . . . . 11

1.2.1. Wavelength calibration . . . . . . . . . . . . . . . . . . . . . . 13

1.2.2. CARMENES . . . . . . . . . . . . . . . . . . 14

1.2.3. Other M-dwarf surveys . . . . . . . . . . . . . . . 16

1.3. Spectral characterisation . . . . . . . . . . . . . . . . . . . 18

1.3.1. Challenges with low-mass stars . . . . . . . . . . . . . 19

2. Methods 23

2.1. The PHOENIX-ACES models . . . . . . . . . . . . . . . . 23

2.2. The fiasco-code . . . . . . . . . . . . . . . . . . . . . . . 24

2.2.1. The fitting procedure . . . . . . . . . . . . . . . . . . . . 24

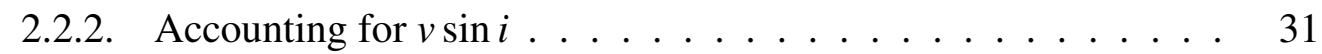

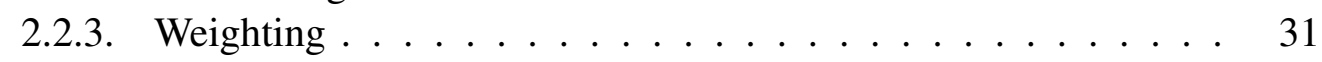

2.2.4. Determination of $\log g \ldots \ldots \ldots \ldots$. . . . . . . . . . . . . . . . . . . . . 31

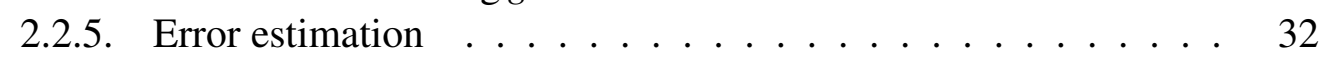

2.3. Spectral regions . . . . . . . . . . . . . . . 33

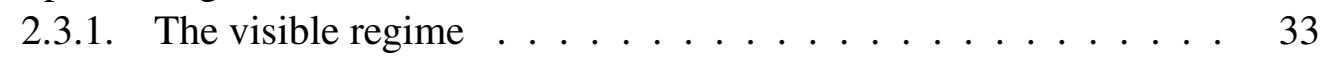

2.3.2. The near-infrared regime . . . . . . . . . . . . . . . . . . 37

2.3.3. Telluric correction ................. . . . 38

$\begin{array}{ll}\text { 3. Data } & 41\end{array}$

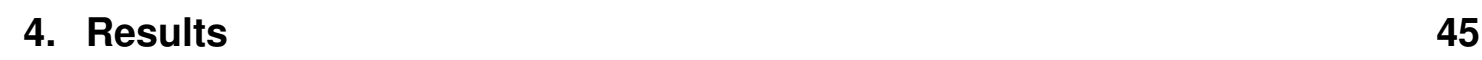

4.1. Results from CAFE, FEROS and HRS . . . . . . . . . . . . . . . . . . . . 45

4.2. Results from CARMENES . . . . . . . . . . . . . . . 50

4.3. Results from TripleSpec . . . . . . . . . . . . . . . . 53

4.4. Results from X-Shooter . . . . . . . . . . . . . . . . . . 57 
5. Discussion 61

5.1. CAFE, FEROS, HRS and CARMENES . . . . . . . . . . . . . . 61

5.2. Comparison of model atmospheres . . . . . . . . . . . . . 69

5.3. TripleSpec and X-Shooter $\ldots \ldots \ldots \ldots$. . . . . . . . . . . . . . . . . .

5.4 . Future work $\ldots \ldots \ldots \ldots \ldots \ldots \ldots \ldots$

6. Summary 75

\begin{tabular}{ll}
\hline A. Appendix: Tables with stellar parameters & 77
\end{tabular} 


\section{List of Figures}

1.1. Planet detections using different techniques . . . . . . . . . . . 2

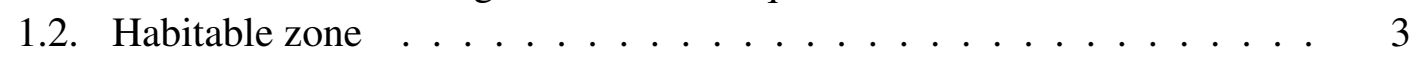

1.3. Description of the planet's orbital elements . . . . . . . . . 5

1.4. Schematic of a lensing event . . . . . . . . . . . . . . 9

1.5. Lightcurve of a lensing event . . . . . . . . . . . . . . . . . . . 10

1.6. Refraction of light from a hot blackbody . . . . . . . . . . . . . . . . . . . . . . . 11

1.7. Schematic of echelle spectrograph . . . . . . . . . . . . . . 12

1.8. CARMENES spectrum of Luyten's star . . . . . . . . . . . . . . . . . . . . 15

1.9. Energy distributions of Sun-like star and M-dwarf . . . . . . . . . . . . 15

1.10. Spatial and spectral type distribution of Carmencita stars . . . . . . . . 16

2.1. Continuum fitting in low-mass stars . . . . . . . . . . . . . . 26

2.2. $\chi^{2}$-maps and curves from first part of fiasco-code . . . . . . . . . . 27

2.3. $\quad$ Best fit from first part of fiasco-code . . . . . . . . . . . . . . . . . . . . . . . . . . . . . 28

2.4. $\chi^{2}$-maps for different fitting algorithms . . . . . . . . . . . . . . . . . . . . . . . . . . . . . . 39

$2.5 . \quad$ Best fit model for GJ $514 \ldots \ldots \ldots$. . . . . . . . . . . . 30

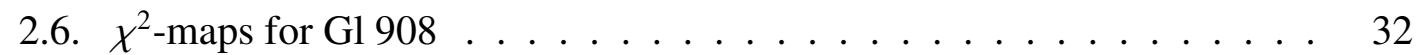

2.7. $\chi^{2}$-maps for GJ 551 in the $T_{\text {eff }}-\log g$ plane . . . . . . . . . . . 34

2.8. $\chi^{2}$-maps for GJ 551 in the $T_{\text {eff }}-[\mathrm{Fe} / \mathrm{H}]$ plane . . . . . . . . . . . . 35

2.9. $\quad$ FEROS spectrum of HD 285968 with modelled telluric lines . . . . . . 36

2.10. TripleSpec spectrum of HIP 12961 . . . . . . . . . . . . . . . . 38

2.11. X-Shooter spectrum of Gl 393 before and after telluric correction. . . . . 40

3.1. SNR distribution for CAFE, FEROS, HRS and CARMENES spectra . . 42

3.2. X-Shooter spectrum of Gl 447 in K-band . . . . . . . . . . . . . . . . 44

4.1. Histograms of CAFE, FEROS and HRS results . . . . . . . . . . . . . 46

4.2. Final result plot of CAFE spectrum . . . . . . . . . . . . . . . . . . . . . . . . . . . 47

4.3. Final result plot of FEROS spectrum . . . . . . . . . . . . . . . . . . . . . . . . . . . . 48

4.4. Final result plot of HRS spectrum . . . . . . . . . . . . . . . . . 49

4.5. Histograms of CARMENES results . . . . . . . . . . . . . . 51

4.6. Final result plot of CARMENES spectrum . . . . . . . . . . . . . 52

4.7. TripleSpec spectrum of Gl 1214 . . . . . . . . . . . . . . . . . 53

4.8. Comparison of TripleSpec results . . . . . . . . . . . . . . . . 56

4.9. X-Shooter spectrum of Gl 285 in NIR . . . . . . . . . . . . . . . 57

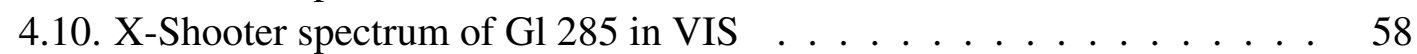

4.11. Comparison of X-Shooter results . . . . . . . . . . . . . 60 
5.1. Comparison of CAFE, FEROS and HRS with CARMENES results . . . 62

\begin{tabular}{lll}
\hline 5.2. & Literature comparison for CAFE, FEROS, HRS and CARMENES results & 65
\end{tabular}

5.3. Comparison of metallicity distributions from CAFE, FEROS, HRS and the solar neighbourhood . . . . . . . . . . . . 66

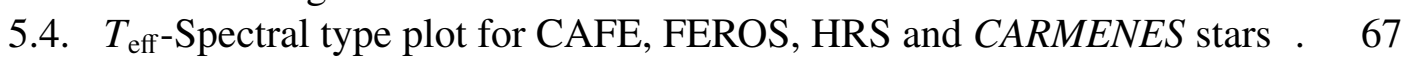

5.5. $\quad$ Mass- $T_{\text {eff }}$ relation for CAFE, FEROS, HRS and CARMENES stars . . . 68

5.6. Comparison of PHOENIX-models with different parameters . . . . . . 71

5.7. CARMENES spectrum of V2689 Ori with two good fitting models . . . 72 


\section{List of Tables}

2.1. Parameter space of the PHOENIX-ACES grid . . . . . . . . . . . . . . 24

2.2. Systematic errors from fitting for stellar parameters . . . . . . . . . . . 33

2.3. Fitting ranges in the NIR . . . . . . . . . . . . . . . . 37

2.4. Wavelength ranges for Molecfit telluric correction . . . . . . . . . . . 39

3.1. Summary of observations . . . . . . . . . . . . . . . . . . . . . . 41

3.2. Sample overlap . . . . . . . . . . . . . . . . . . . . 42

4.1. Results from TripleSpec spectra in the J-, H- and K-bands . . . . . . . . 55

4.2. $\quad$ Results from X-Shooter spectra in the J-, H-bands and VIS . . . . . . . 59

A.1. Measured astrophysical parameters of investigated stars . . . . . . . . . 78

\begin{tabular}{lll}
\hline A.2. Measured astrophysical parameters of investigated stars without $v \sin i$ & 93
\end{tabular} 



\section{Introduction}

The main goal of this work is the development of a method to derive accurate fundamental stellar parameter of $\mathrm{M}$ dwarfs from their spectra in the framework of planetary system characterisation. In the following sections I will give an introduction to the different methods for detecting exoplanets, the CARMENES project and the challenges of spectral characterisation, especially for low-mass stars.

\subsection{Exoplanets}

Mankind has always been fascinated by the idea of extraterrestrial life on planets outside of our solar system. Looking at the millions of stars in our Milky Way, the possibility of having other planets orbiting these stars cannot be denied. Already in 1584 Giordano Bruno said:

"There are countless suns and countless earths all rotating round their suns in exactly the same way as the seven planets of our system. (...) The countless worlds in the universe are no worse and no less inhabited than our earth. For it is utterly unreasonable to suppose that those teeming worlds (...) should be uninhabited and should not bear similar or even more perfect inhabitants than our earth." (from Bettex, 1965)

Despite uncountable science fiction scenarios about other worlds, scientists developed more and more advanced technologies and instruments, so finally in 1992 the first extrasolar planet candidate was detected. Wolszczan \& Frail (1992) found timing variations in the radio pulses of the millisecond pulsar PSR B1257+12. Although several exoplanets have already been claimed in the late 1980s and early 1990s (e.g. Campbell et al. 1988, Hatzes \& Cochran, 1993), the first confirmed exoplanet was found by Mayor \& Queloz (1995) using the radial-velocity (RV) method. 51 Pegasi b orbits a Sun-like star with a period of 4.2 days. Since then the number of detections has increased every year. By the end of 2016, 6177 planets were claimed, 3557 of them confirmed (exoplanet.eu). Most of them have been found using radial-velocity measurements and transit photometry. This work uses spectroscopic observations and serves as a preparation for the CARMENES radial-velocity survey. However, there are several other methods for detecting exoplanets. Fig. 1.1 presents the masses of exoplanets detected with different techniques as a function of stellar mass. So far, only a few sub-Jupiter mass planets have been found around $\mathrm{M}$ dwarfs $\left(\mathrm{M} \lesssim 0.7 \mathrm{M}_{\odot}\right)$, which encourages next generation planet search projects to close this gap. In the following sections I will briefly describe the habitable zone and different methods for exoplanet detection. A more detailed study is presented by Perryman (2011). 


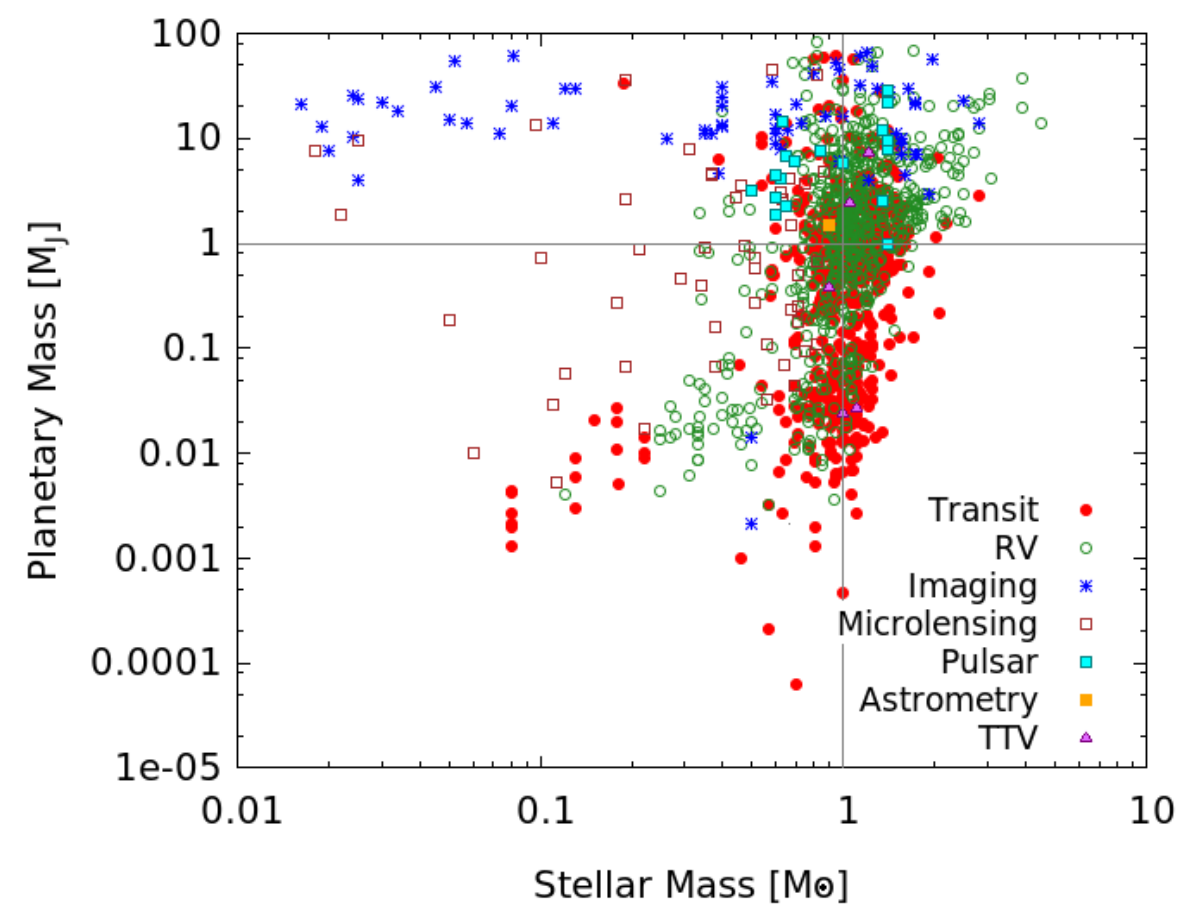

Figure 1.1.: Planet detections using different techniques. Only a few sub-Jupiter mass planets have been detected around low-mass stars so far, mainly from transit and radialvelocity (RV) observations.

\subsubsection{Habitable zone}

The detection of exoplanets showed that other worlds indeed exist in our galaxy. As a consequence the interest in finding habitable planets increased. A planet is considered habitable if the surface temperature is within the range where liquid water can exist, which is believed to be the key requirement to develop life as we know it. To fulfil this requirement the planet has to orbit within a certain zone around its host star, called the "habitable zone". Huang (1959) first brought up the concept of the habitable zone. The classical habitable zone for main sequence stars was defined by Kasting et al. (1993). A diagram is shown in Fig. 1.2. They defined the width of the habitable zone around main sequence stars assuming a planetary atmosphere similar to Earth with carbon-dioxide, water and nitrogen. The loss of water caused by hydrogen escape and photolysis determines the inner edge of the zone. At the outer edge $\mathrm{CO}_{2}$ clouds are formed, which increase the planet's albedo and cool down its surface. For the solar system these estimates transfer to a habitable zone at distances between 0.95 and 1.37 AU from the Sun. However, the actual width of the habitable zone depends on many factors, e.g. the stellar spectral type and age, the planetary size and rotation, the orbital eccentricity, the presence of greenhouse effect and internal heating, atmospheric composition and circulation. The stellar spectral type is important in two ways. First, it constrains the wavelength where most of the stellar flux is emitted, and therefore the stellar temperature. The later the spectral type and lower the temperature of the star, the closer the habitable zone is to the star. Second, it also defines the lifetime of the star and conse- 


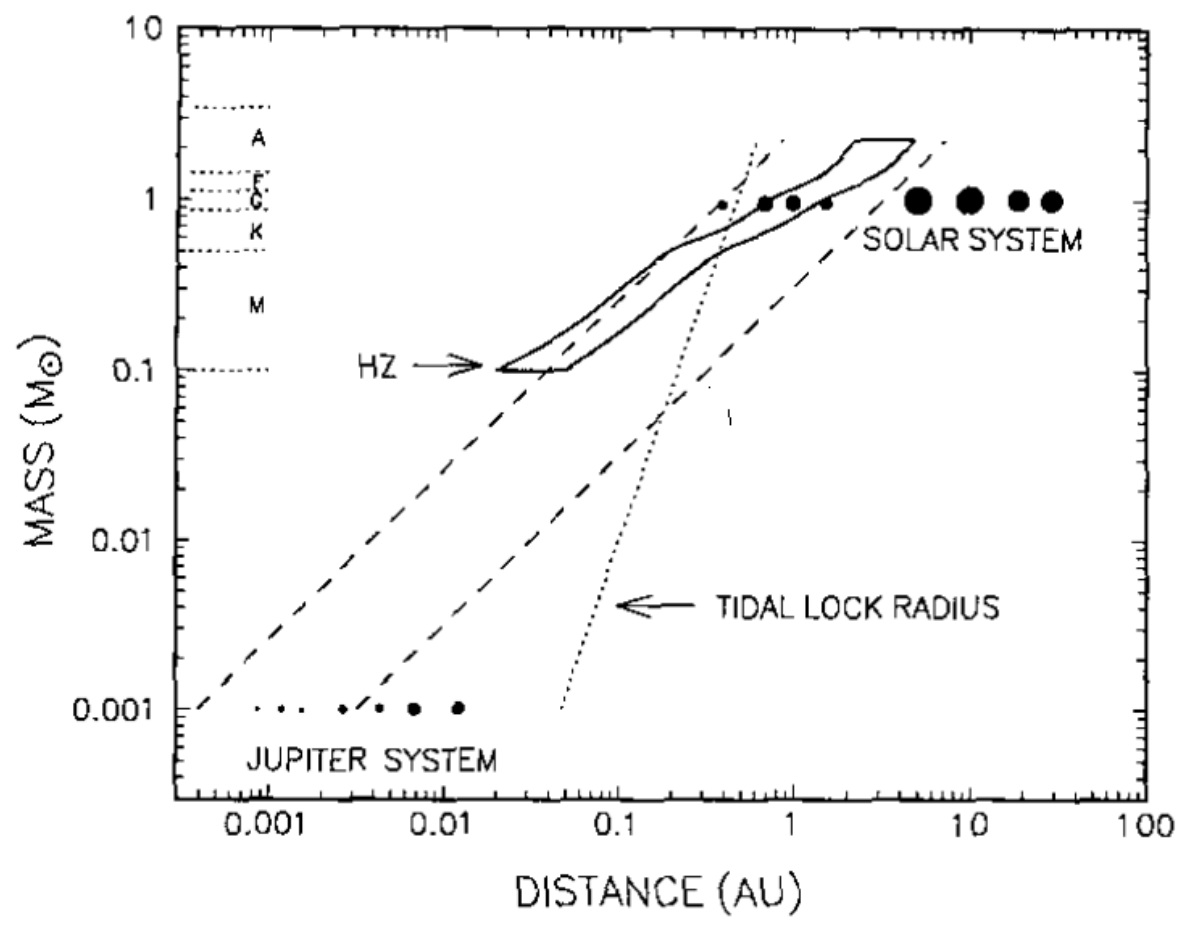

Figure 1.2.: Plot showing the stellar mass as a function of the planet's distance to the star in AU. The habitable zone (HZ) is indicated by the solid lines. Spectral types are written on the left. Image credit: reprinted from Kasting et al. (1993), Fig. 16, with permission from Elsevier.

quently if life on the planet has enough time to evolve. Given that life needs at least 3.7 billion years to evolve (shown by the oldest fossils found on Earth, see Nutman et al., 2016), stars with spectral types earlier than F are not suitable. Even F-type stars might evolve too rapidly to support the evolution of intelligent life. Late $\mathrm{K}$ and, especially, $\mathrm{M}$ dwarfs have lifetimes of up to 13 Gyrs. However, they may not be suitable for life either. The habitable zones around these stars lie within the tidal lock radius, so the planet will face the star always with the same side. However, this does not necessarily mean that the planet is inhabitable. If there is sufficient atmospheric heat transport (Haberle et al., 1996) to avoid freezing on the night-side and a strong enough magnetic field (Tarter et al., 2007) to prevent the loss of the planet's atmosphere, the planet might be habitable. Also, most $\mathrm{M}$ dwarfs are highly active, having a high rate of coronal mass ejections and strong ultraviolet radiation (Buccino et al., 2007), which is known to damage biological systems and destroy DNA. Here again a strong magnetic field could protect the planet from stellar UV radiation. The planetary properties can extend the outer boundary of the habitable zone, whereas the inner edge was found to be rather constant by runaway greenhouse effect and therefore water loss (Kasting, 1988). The radius of the planet is also important for its habitability. With too small of a radius, i.e. mass, the gravitation is too weak to keep the planetary atmosphere. Also plate tectonics stop, which are thought to be an important part in the $\mathrm{CO}$ cycle due to the presence of volcanic activity. If the planet is too massive, it will start accreting $\mathrm{H}$ and $\mathrm{He}$ to become a gas giant. The limits 
in planet size lie between $0.8-2.2 R_{E}\left(0.5-10 M_{E}\right.$, Perryman, 2011).

\subsubsection{Radial-velocity method}

In a planetary system all bodies, including the star, orbit around the center of mass. The stellar motion causes a periodic red- and blue-shift of the star's spectral lines, which can be detected. The maximum shift of the spectral lines is called Kepler amplitude and is defined as

$$
K=\left(\frac{2 \pi G}{P}\right)^{1 / 3} \frac{M_{p} \sin i}{\left(M_{s}+M_{p}\right)^{2 / 3}} \frac{1}{\left(1-e^{2}\right)^{1 / 2}}
$$

with $G$ being the gravitational constant, $P$ the period of the planet, $M_{p}$ the planetary mass, $M_{s}$ the stellar mass, $e$ the eccentricity of the orbit, and $i$ the inclination of the orbit with respect to the line of sight.

A Keplerian orbit can be described by 7 parameters, which are the semi-major axis $a$, the eccentricity $e$, the period $P$, the inclination $i$, position of the planet on its orbit at a certain time $t_{p}$ usually with respect to the pericenter passage (described as time-dependent angle $v(t)$ ), the argument of pericenter relative to the ascending node $\omega$ and the longitude of the ascending node $\Omega$. From radial-velocity measurements alone, only a combination of $M_{p} \sin i$ and $a \sin i$ can be derived. Other directly observable parameters are $P, e, t_{p}$ and $\omega$. Fig. 1.3 depicts the planetary orbit and all its elements described above.

Equation 1.1 shows that larger planets on closer orbits to the star produce a larger signal. Because of that radial-velocity detections up to now are biased towards this regime. New-generation spectrographs with higher precision will extend the parameter space. The signal also increases towards lower masses of the host star. This and the fact that the habitable zone moves closer to the star with lower luminosity, brought $\mathrm{M}$ dwarfs more and more into focus of planet searches (e.g. CARMENES see Section 1.2.2). In general, the RV signal of Jupiter around the Sun is $12.5 \mathrm{~ms}^{-1}$, whereas for Earth the signal is only $0.09 \mathrm{~ms}^{-1}$. A $2 \mathrm{M}_{\oplus}$ planet in the habitable zone (about $0.15 \mathrm{AU}$ ) around an M4 star gives a signal of about $0.9 \mathrm{~ms}^{-1}$ and therefore could be detectable with upto-date spectrographs, which aim for a detection limit in the $1 \mathrm{~ms}^{-1}$ regime and below (see Section 1.2). First M-dwarf radial-velocity surveys have already started in the early 2000s, e.g. Endl et al. (2003), Kürster et al. (2009).

However, several aspects need to be taken into account to reach these low limits. Effects which contribute to the measured radial-velocity curve are the Earth's motion around the Sun, the star's radial motion through space, line shifts due to stellar convection, spots and the star's gravitational field. Another crucial aspect is the wavelength calibration. In order to detect a radial-velocity shift the wavelengths of the lines in the rest frame need to be known with high accuracy. This can be achieved by using different types of lamps (e.g. iodine, thorium-argon), laser-frequency combs or Fabry-Pérot etalons (see Section 1.2.1. 


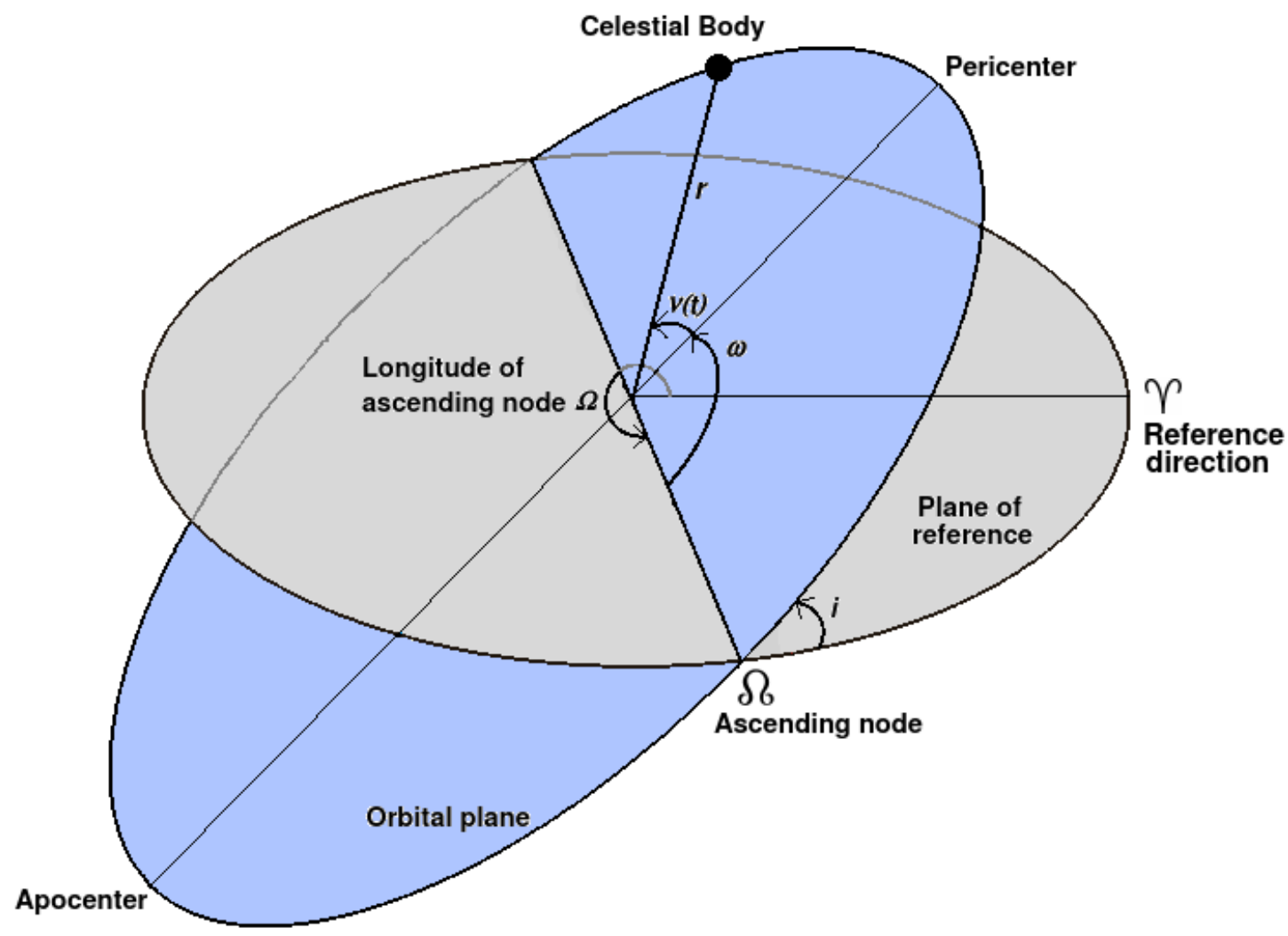

Figure 1.3.: Description of the planet's orbital elements.

\subsubsection{Transit method}

The first exoplanet transit detected was HD 209458 by Henry et al.(1999). Until December 30, 2016, 2697 transiting planets have been detected from ground- and space-based observatories (exoplanet.eu). With suitable alignment of the planetary orbit with respect to the observer's line of sight, the planet crosses the stellar disk periodically. The drop in stellar flux $\Delta F$ can be measured and is directly proportional to the size ratio of star and planet

$$
\Delta F=\left(\frac{R_{p}}{R_{s}}\right)^{2}
$$

where $R_{p}$ and $R_{s}$ are the planetary and stellar radii, respectively.

The effect is very small, the drop in flux for a Sun-like star orbited by a Jupiter-like planet is about 1 percent. This favours transit searches around low-mass stars in order to detect Earth- or Super-Earth-sized planets, e.g. the MEarth project (Charbonneau et al., 2008, see Section 1.2.3. To be able to observe a transit, the inclination angle of the orbit is very important. It depends on the stellar radius and the planet's semi-major axis and eccentricity. The minimum angle is usually close to 90 degrees and defined by

$$
\cos i_{\text {min }}=\frac{R_{s}}{a} \frac{1}{1-e^{2}}
$$


where $a$ is the planet's semi-major axis and $e$ the eccentricity of the orbit.

From a transit lightcurve, the main observables are the transit depth, length and period. Having an estimate about the stellar mass and radius, e.g. from spectroscopy, the planetary radius and the semi-major axis can be derived. Seager \& Mallén-Ornelas (2003) present a unique solution of planetary and stellar parameters. This solution exists under the assumptions of a circular orbit, negligible limb-darkening for observations at infrared wavelengths and ignoring all possible contaminating sources (e.g. background eclipsing binaries). With this and assumptions about the stellar mass-radius relation six physical parameters can be derived: $M_{s}, R_{s}, R_{p}, i, a$ and the stellar density $\rho_{s}$. For eccentric orbits this solution does not hold anymore and more complex equations need to be applied. The eccentricity itself can be theoretically derived from the different transit ingress and egress times. In general, this effect is hard to detect and additional radial-velocity measurements are necessary.

There are several other phenomena that deform the transit lightcurves and theoretically can be detected. Those include star spots, planetary rings or moons, and other non-transiting planets (from transit timing variations, see Section 1.1.6). Using transit spectroscopy, the planetary atmosphere can be investigated when the stellar light shines through the atmosphere during transit.

\subsubsection{Direct Imaging}

In this method an image of the planet is obtained, with the main goal to prove its existence. It can be observed either in visible wavelengths due to reflected light from the host star, or in the infrared due to the thermal emission of the planet itself. The major problems with this method are the small star-planet brightness ratios and the small angular distances between star and planet. The brightness ratio depends on many properties, like the spectral type and luminosity of the star, the size, age and composition of the planet as well as its atmospheric scattering properties and its semi-major axis. Typical ratios lie between $10^{-5}$ in the infrared and $10^{-10}$ in the optical wavelength range. The angular distance of the planet depends on the current position on its orbit and on the distance of the system to the observer. Typical values range from $0.1-0.5 \operatorname{arcsec}$ and therefore the planet lies within the stellar glare. Several techniques try to improve observations and reduce disturbing effects to make a planet detection via imaging possible.

With observations from the ground, the "seeing" caused by atmospheric turbulences is the major issue. To compensate for that adaptive optics are used. A sensor analyses the distorted wavefronts and a correction is applied by deforming several movable mirror segments of the telescope. This technique allows for corrections in the order of $1 \mathrm{~ms}$. For analysing the wavefront a bright star near the target star is necessary. Because bright stars are rather rare on the sky, laser guide stars are employed. The lasers ionise the atmospheric sodium layer at an altitude of $80-100 \mathrm{~km}$ nearby the target star. This gives a point light source that acts as an artificial star for wavefront correction.

To reduce the stellar light that outshines the planet, coronographic masks are used. Those either block the stellar light by introducing a physical mask in the focal plane of the telescope (e.g. Lyot coronography, Lyot, 1939, Malbet, 1996) or cancel the light through self-interference by the use of phase masks (e.g. four-quadrant phase mask, Rouan et al. 
(2000); optical vortex coronograph, Foo et al. (2005), Swartzlander et al. (2007)).

A prominent image of an exoplanet is the one from Fomalhaut b. Fomalhaut is a mainsequence star of spectral type A3 surrounded by a debris disk. With observations from Keck and Gemini, Kalas et al. (2009) showed evidence of a less than 3 Jupiter-mass planet orbiting at the inner edge of the debris disk at about 115 AU.

\subsubsection{Astrometry}

The motion of star and planet around the barycenter causes a physical displacement of the star on the sky. Projected on the sky plane this motion describes an ellipse with semi-major axis

$$
\alpha=\left(\frac{M_{p}}{M_{s}}\right)\left(\frac{a}{1 A U}\right)\left(\frac{d}{1 p c}\right)^{-1} \operatorname{arcsec}
$$

where $d$ is the distance between observer and system.

Other than radial-velocity measurements, which yield only an estimate of the planetary mass $M_{p} \sin i$, because the orbital inclination $i$ can not be determined separately, the astrometrical method provides the planetary mass directly if the stellar mass is known. As can be seen from this equation, astrometry is very sensitive to near-by stars with planets in long-period orbits. The size of the effect can be rather small, depending on the planetary mass. Seen from a distance of $10 \mathrm{pc}$, the Earth would introduce a signature of $0.3 \mu$ as, whereas Jupiter would give a signal of $500 \mu$ as.

There are many effects that make it difficult to detect these small signals. For observations from the ground the main limit is atmospheric seeing, which is typically around 1-3 arcsec. This limit can be pushed using adaptive optics, so that short-term precisions of around $100 \mu$ as have been achieved with the near-infrared imager NACO on VLT (Neuhäuser et al., 2007). Up-to-date space missions like GAIA demonstrate precisions of around $24 \mu$ as $(\overline{E S A}, 2016)$. In the $\mu$ as-regime, higher-order physical effects gain influence. Examples for those are the stellar motion through space, light deflection by the Sun's or Jupiter's gravitational field, the Earth's motion around the solar system barycenter, and "jitter" from the stellar surface caused by spots, granulation or oscillations.

\subsubsection{Timing}

As explained in Section 1.1.2, the star and the planet orbit around the center of mass. If the star produces a periodic signal, e.g. a pulsar, the change in the signal's period can be measured due to light travel time. The amplitude of this change is described by

$$
\tau_{p}=\frac{1}{c} \frac{a \sin i M_{p}}{M_{s}}
$$

where $c$ is the speed of light and $a$ the semi-major axis of the orbit. 
Any stellar object possessing periodic time signals can be used for detection. These are pulsars, pulsating stars and eclipsing binaries. Pulsars are fast rotating highlymagnetised neutron stars, emitting narrow beams of radio emission along their magnetic dipole axis. Because of a misalignment of the spin and the magnetic axes the beams can be observed as pulses at the star's rotation period. Pulsars are classified as "normal" pulsars with rotation periods of around 1 second, or as "millisecond" pulsars with periods in the millisecond regime. The latter ones are very suitable for planet detections, because their period is stable within ns (e.g. PSR J0437-4715, van Straten et al., 2001). The first exoplanet was detected around the millisecond pulsar PSR B1257+12 (Wolszczan \& Frail, 1992). Until the end of 201624 planets have been confirmed around pulsars (exoplanet.eu).

White dwarfs can also be used for timing methods. Some of them are the most stable pulsators known, e.g. G117-B15A with a stability of around $10^{-15} \mathrm{~s} \mathrm{~s}^{-1}$ (Kepler et al., 2005). During their cool-down phase, $\mathrm{C} / \mathrm{O}, \mathrm{He}$ and $\mathrm{H}$ become partially ionised in the photosphere and generate multi-periodic non-radial g-mode pulsations. They have pulsation periods between 100-1000 s.

Planets around eclipsing binaries can be detected due to the varying eclipse times of the binary system. These transit-timing variations (TTVs) can also be used to detect additional, non-transiting planets from the transit lightcurves of known planets in a system. In 2012, the first circumbinary planetary system was detected around Kepler-47AB (Orosz et al., 2012). The binary stars have a period of 7.45 days. The inner planet $b$ has a period of 49.51 days and a radius of about 3 Earth-radii. The outer planet $\mathrm{c}$ is larger with about 4.6 Earth-radii and a period of 303.16 days.

\subsubsection{Microlensing}

The gravitational potential of a foreground object can bend light from a distant background object if both objects happen to be in favourable alignment with the observer. In this context the foreground object acts as a lens and the background object is considered as the source. The result are (often multiple) distorted images of the source, which can be focused and therefore amplified. The amplification varies with time due to the motion of observer, lens and source. The lightcurve also changes depending on the complexity of the lens, which makes it possible to detect orbiting planets from additional magnifications. Because these events need a precise alignment (within the angular Einstein radius, see Equation 1.8 between observer, lens and source, the probability for one event is about $10^{-8}$. In surveys, large numbers of stars, preferable towards the Galactic bulge region where the stellar surface density is high, are monitored simultaneously. Fig. 1.4 shows a schematic of a lensing event, an observed lightcurve caused by the lensing event of OGLE-2005-BLG-071 is pictured in Fig. 1.5. The additional magnifications peaks reveal the presence of a planet, where the three cusps of the stellar caustic lead to the triple peak shape of the magnification. The event was discovered by Udalski et al. (2005) and further analysed by Dong et al. (2009), who claimed a stellar mass of $0.46 \pm 0.04 M_{\odot}$ and a planetary mass of $3.8 \pm 0.4 M_{J}$.

From the geometry several equations can be derived to describe the event. The Schwarz- 


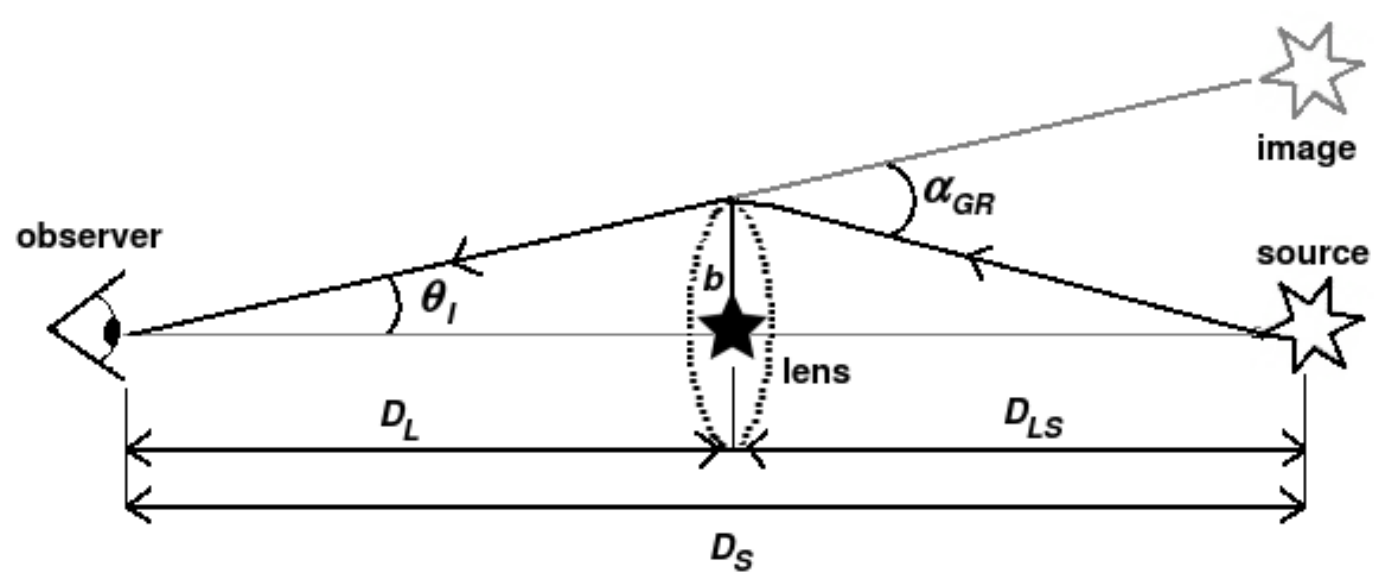

Figure 1.4.: Schematic of a lensing event for a lens with mass $M_{L}$ at distance $D_{L}$ from the observer. The source is located at a distance $D_{S}$ from the observer and at a distance $D_{L S}$ from the lens. The light from the source is deflected under the angle $\alpha_{G R}$ and creates an image, which is seen under the angle $\theta_{I}$ by the observer.

schild radius $R_{S}$ depends on the mass $M_{L}$ of the lensing object

$$
R_{S}=\frac{2 G M_{L}}{c^{2}}
$$

The Schwarzschild radius defines the radius of a collapsed object, where the escape velocity from the surface would be equal to the speed of light. It can be used to describe the deflection angle $\alpha_{G R}$

$$
\alpha_{G R}=\frac{2 R_{S}}{b}=\frac{4 G M_{L}}{c^{2} b}
$$

with the impact parameter $b$.

The characteristic angle $\theta_{E}$, the angular Einstein radius, and the characteristic length $R_{E}$, the linear Einstein radius, are defined by

$$
\begin{aligned}
\theta_{E} & =\left(2 R_{S} \frac{D_{L S}}{D_{L} D_{S}}\right)^{1 / 2} \\
R_{E}=\theta_{E} D_{L} & =\left(2 R_{S} \frac{D_{L} D_{L S}}{D_{S}}\right)^{1 / 2}
\end{aligned}
$$

From the lightcurve the geometry and the mass distribution of the system can be inferred. The first detection of an exoplanet using the microlensing method was reported in 2004 by Bond et al. (2004). By the end of 2016, 52 planets have been found by this method (exoplanet.eu). 


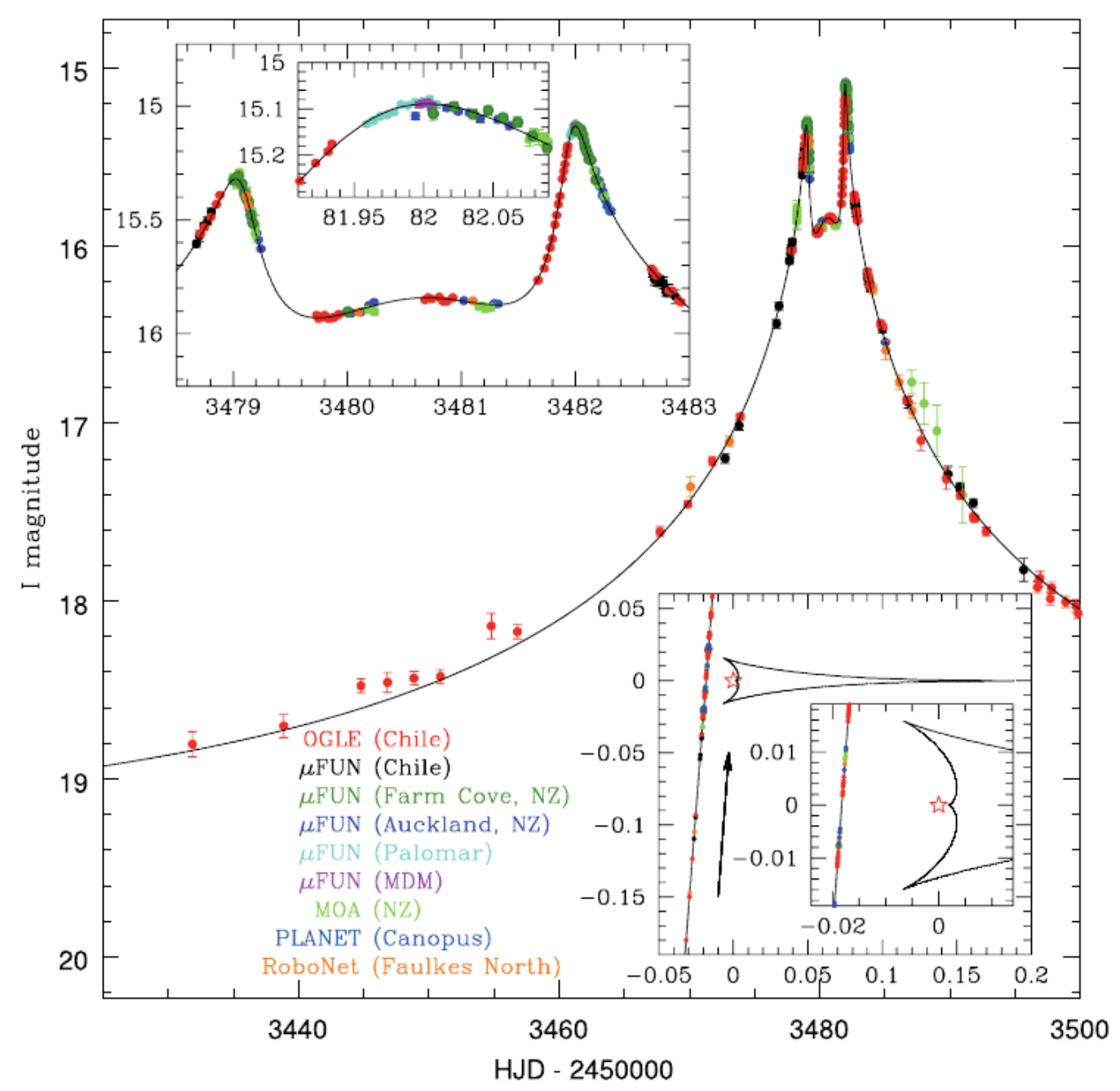

Figure 1.5.: Example of a typical microlensing lightcurve of OGLE-2005-BLG-071. The upper insets show a zoom-in on the planetary anomaly near the peak, the lower insets illustrate the path of the source passing near the stellar caustic. The three cusps of the caustic are represented by the triple peak in the lightcurve. Image credit: Udalski et al. (2005), Fig. 1, reproduced with permission of the AAS. 


\subsection{Spectroscopy}

In the following I will focus more on spectroscopy with view on radial-velocity measurements and surveys, especially CARMENES, but also with a short overview on other $\mathrm{RV}$ planet search projects. In the end of this section the challenges with spectroscopic investigation of low-mass stars will be discussed.

Investigating the electromagnetic spectrum of a light source or hot medium emitting electromagnetic radiation is a powerful tool to study the chemical composition of the medium. White light can be split into its different wavelength components by a simple glass prism because the refraction angle is wavelength dependent. The result is a multicolour spectrum (case a) as shown in Fig. 1.6.

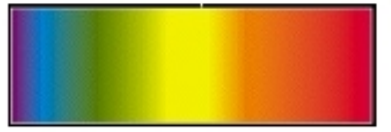

a) Continuous spectrum

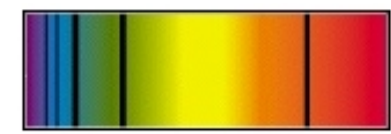

b) Absorption line spectrum

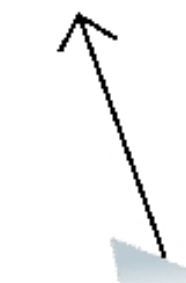

Prism

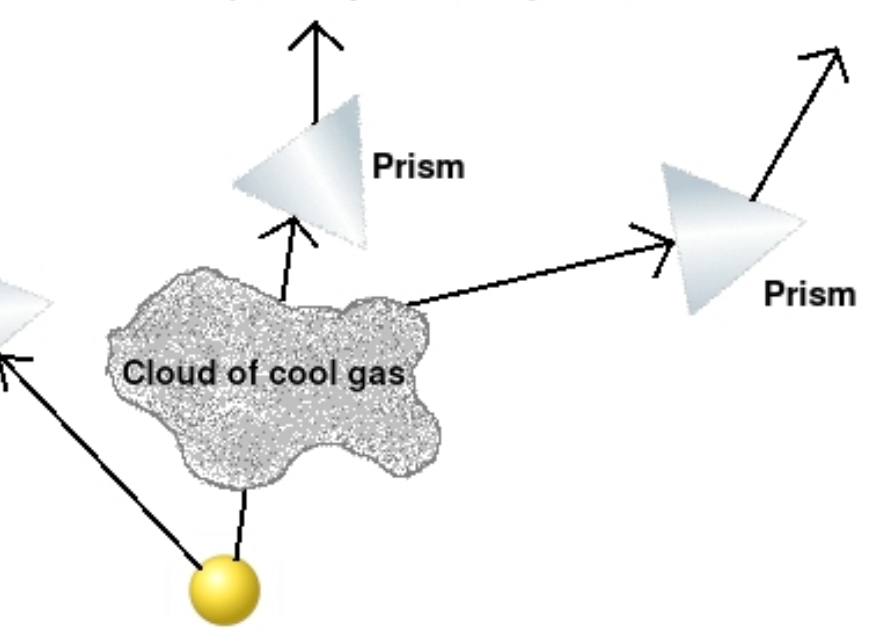

) Emission line spectrum

\section{Hot Blackbody}

Figure 1.6.: Refraction of light from a hot blackbody into a) a continuous spectrum, b) absorption spectrum when observed through an absorbing medium, and c) emission spectrum of the medium itself.

An absorption spectrum (case b) is a spectrum of white light containing dark lines, where photons of a certain energy were absorbed by a medium between the light source and the observer. The absorbing medium can be identified from these lines since every element has its one characteristic absorption spectrum.

In contrast to that is the emission spectrum (case c). Emission occurs when an electron in an atom decays from a higher energy state to a lower one by emission of a photon. The energy of the photon is equivalent to the energy difference between the two states, resulting in a spectral line of a certain wavelength. Since many atoms of the same species can be in different excited states and relax to different lower states, several spectral lines of different energies are produced. The atoms can be identified from the wavelengths of 
their spectral lines, which are known from laboratory experiments and calculations.

The first studies of the solar spectrum have been made in the 17 th century by Kircher (1646), Marci (1648), Boyle (1664) and Grimaldi (1665), prior to the work done by Newton (1672), and all of them using a simple prism to split the solar light into spectral lines. In the 19th century, more than a hundred years before the first confirmed exoplanet detection, Niven (1874) proposed the possibility of observing the radial-velocity shift of a star induced by an orbiting planet.

The design of the spectrograph changed over the centuries and especially the last decades. First, simple glass prisms were used, as described by Kircher (1646) and the other authors of the 17th century mentioned before. Newton (1730) already introduced a slit of 0.1 inch to increase the resolution of the spectrum. The first collimator lens was used by Simms (1840). The spectrographs got more and more advanced, e.g. by using different arrangements of prisms and lenses, but prisms were used until the early 20th century. Waterhouse (1890) and later Ames (1892), Wadsworth (1895) and Wadsworth (1898) describe the use of a grating in spectrographs. Michelson (1898) already explained the principles of an early form of echelle spectrograph (under the name echelon spectroscope), using a grating at grazing angles.

The echelle spectrograph uses an echelle grating, a plane reflection grating with a blaze angle of typically $60^{\circ}$. The blaze angle defines the angle by which the reflective steps are inclined with respect to the grating plane. The light is split into multiple orders with high order number that overlap each other (e.g. Porter, 2000). A cross disperser, with the dispersion direction orthogonal to the echelle grating, is mounted into the beam path to separate the different orders before detection on the Charge-Coupled Device (CCD). These spectrographs are operated until today since they are able to provide large wavelength coverage. A schematic of an echelle spectrograph is shown in Fig. 1.7 .

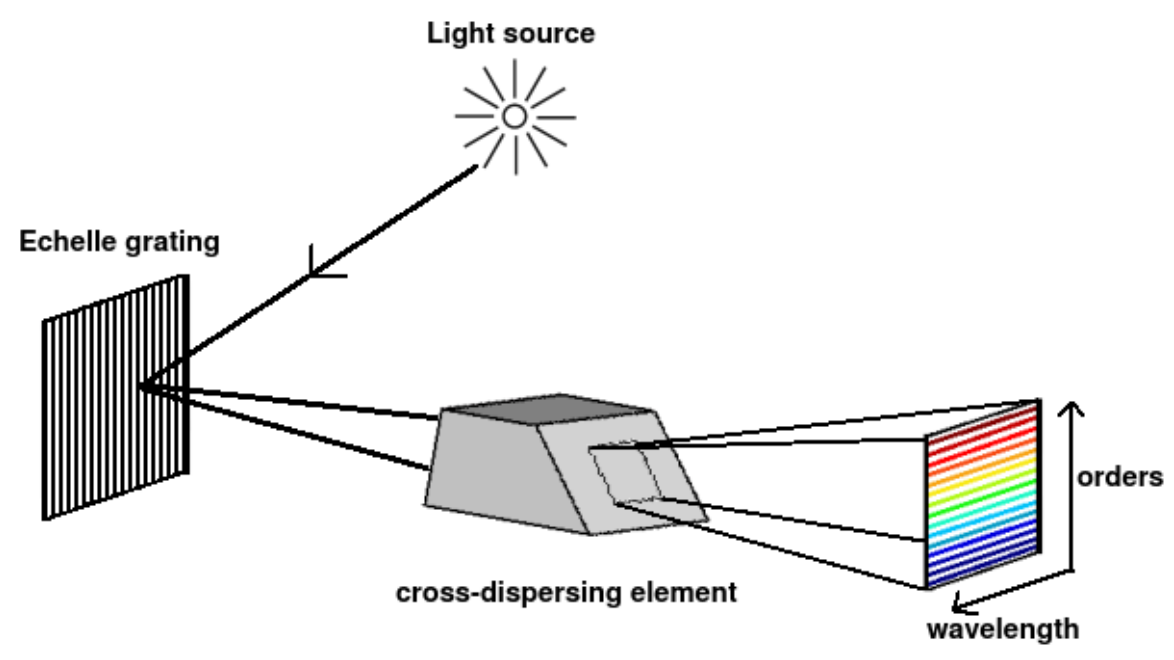

Figure 1.7.: Schematic of an echelle spectrograph. 


\subsubsection{Wavelength calibration}

The wavelengths of a spectral line cannot be directly measured on the CCD; only the pixel position is known. To translate the pixel position into absolute wavelength, the spectrograph system has to be calibrated. This is done by obtaining a reference spectrum of a medium where the wavelength of each spectral line is known. Several devices can be used for that purpose, e.g. iodine absorption cells, hollow-cathode lamps (HCL), Fabry-Pérot interferometers or laser frequency combs.

Iodine absorption cells (e.g. Marcy \& Butler, 1992) are very simple and low cost calibrators. The absorption spectrum contains a high density of lines between 5000 and $6300 \AA$. The cells are long-term stable and need almost no maintenance.

Most echelle spectrographs today use HCLs. The lamps consist of a hollow metal cylinder, typically thorium or uranium, filled with a noble gas like argon or neon (Kerber et al., 2007). The gas atoms are exited by collisions with electrons that migrate from the cathode to the anode. The exited gas atoms collide with the cathode and excite the thorium or uranium atoms, which emit photons by relaxing into a lower energy state. The wavelengths of these emission lines are well known from measurements and calculations (e.g. Lovis \& Pepe, 2007, Redman et al., 2014). However, since the precision of up-to-date and future spectrographs increases, HCLs have become insufficient because of their limited number of lines, irregular distribution and blends of lines.

A Fabry-Pérot interferometer provides a dense grid of lines with regular distribution over the whole spectral range. It consists of two semi-transparent mirrors, where the light rays enter on one side, are reflected between the mirrors and interfere with each other, and are transmitted to the other side, where they show an interference pattern. Fabry-Pérot etalons can have different designs either using a single glass plate with two reflecting surfaces, a glass fibre with coating on both ends, or two mirrors in a mount. The wavelength of each interference peak depends on the distance between the mirrors or the reflecting surfaces. The wavelength solution obtained from HCLs can be used to estimate this distance. The combination with HCLs makes Fabry-Pérot etalons into high-precision wavelength calibrators, as shown by Bauer et al. (2015).

A relatively new technique are frequency combs generated from mode-locked femtosecond-pulsed lasers (e.g Braje et al., 2008, Murphy et al., 2007, Wilken et al., 2010). The laser produces light pulses of a few femtoseconds which are fed into a high dispersive fiber. The fiber performs a Fourier transformation on the continuous wave trains, which results in a spectrum of unresolved lines that are equidistant in frequency space depending on the frequency of the laser. The frequency can be locked to an extremely precise source like an atomic clock. Together with a reference line (e.g. from ThAr) the frequencies can be translated into wavelengths. This technique provides a wide spectral coverage with long-term stability and precisions down to the $\mathrm{cms}^{-1}$ regime, but is also of much higher cost than Fabry-Pérot interferometers. Since the Fabry-Pérot interferometer combined with HCLs provide a high-precision wavelength calibration together with low acquisition costs, this system is used for the CARMENES spectrographs. 


\subsubsection{CARMENES}

The Calar Alto high-Resolution search for M dwarfs with Exo-earths with Near-infrared and optical Echelle Spectrographs (hereafter CARMENES) is a joint project between 11 German and Spanish institutions. The instrument is installed at the Zeiss $3.5 \mathrm{~m}$ telescope at Calar Alto Observatory, located in the Sierra de Los Filabres, Almería, in southern Spain. The two fibre-fed spectrographs operate in the visible wavelength range from $0.55-0.95 \mu \mathrm{m}$, and in the near-infrared from $0.95-1.7 \mu \mathrm{m}$ with a resolution of $R \sim 82000$. They are constructed for high long-term stability, which allows for radialvelocity measurements with high precision of about $1 \mathrm{~ms}^{-1}$ (Quirrenbach et al., 2010, 2012, 2014). Fig. 1.8 shows the spectrum of Luyten's star from both spectrographs. During the project, which started in January 2016, around $300 \mathrm{M}$ dwarfs are being observed with the aim of detecting planets in the habitable zone of their host stars. The main focus lies on low to moderately active stars with spectral types later than M4 V. Using Equation 1.1 together with the mentioned precision of around $1 \mathrm{~ms}^{-1}$ shows that it should be possible to detect a 2-Earth-sized planet in the habitable zones $(0.1 \mathrm{AU})$ of M3 V stars $\left(0.3 R_{\odot}\right)$. In addition, the target stars are located very nearby in the solar neighbourhood, which allows further characterisation of potential planets. The simultaneous observations in the visible and near-infrared are advantageously for distinguishing between a signal caused by a planetary companion or stellar activity. It was shown by Martín et al. (2006) that stellar activity seems to have less impact on the RV amplitude in the near-infrared compared to the visible, whereas a planet would give the same signal in both channels. Another reason for observing in the near-infrared is that for cooler stars the overall energy distribution shift towards longer wavelengths, peaking in the nearinfrared for M dwarfs. Fig. 1.9 shows the energy distributions for a Sun-like star and a mid-M dwarf. A huge amount of time has been invested to collect information about the target sample, either from literature or from new low- and high-resolution spectroscopy and imaging. This ensures that the CARMENES guaranteed time of 600 clear nights is spent in the most efficient way. Alonso-Floriano et al. (2015) present results obtained from low-resolution spectroscopy concerning spectral types and activity, whereas Jeffers et al. (submitted), Passegger et al. (in prep.) and this work focus on science preparation using high-resolution spectroscopy. All information about the target stars is collected in the CARMENES Cool dwarf Information and daTa Archive (hereafter Carmencita). A very detailed description of the Carmencita database was done by Cortes-Contreras (2016). The archive contains parameters of approximately $2200 \mathrm{M}$ dwarfs, including accurate distances, rotational, radial and Galactocentric velocities, photometry in 20 bands from the ultraviolet to the mid-infrared, spectral types, effective temperatures, surface gravities, metallicities, $\mathrm{H} \alpha$ emission, $\mathrm{X}$-ray count rates and hardness ratios, membership in open clusters and young moving groups, information about close and wide multiplicity, target in other RV surveys, and exoplanet candidacy (Caballero et al., 2013). Target stars have been selected according to two criteria. First, they need to be observable from Calar Alto Observatory, having declinations $\delta>-23 \mathrm{deg}$, and second, the brightest stars for each spectral type have been selected. The faintest targets in the catalogue are of $\mathrm{J}=11.5 \mathrm{mag}$. For selecting stars for the final target list, the potential targets have been divided into four priority groups Alpha, Beta, Gamma and Delta. The Alpha group con- 
tains stars with highest priority, which are the brightest ones for each spectral subtype. These are around 300 stars, as mentioned before. Beta stars are still relatively bright and serve as backup. The faintest stars are collected in the Gamma group, which is the majority of all potential targets. Finally, the Delta group consists of stars with physical or visual companions within 5 arcsec, whose light could contaminate the spectra of the target stars. Fig. 1.10 shows the distribution of Carmencita stars on the sky and as a function of spectral type.

With this work, fundamental parameters of the target stars are determined, which will help to characterise potential planets found with CARMENES.

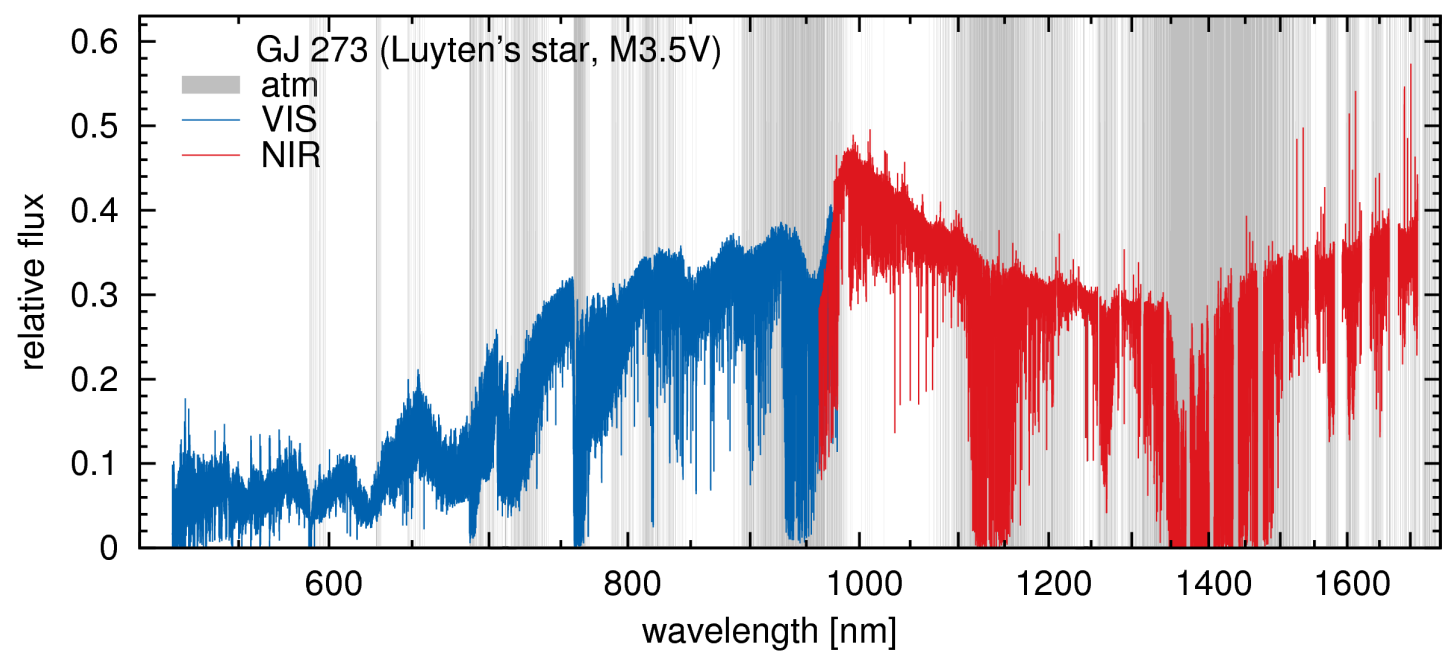

Figure 1.8.: Complete CARMENES spectrum of Luyten's star (Gl 273) for the visible (blue) and the near-infrared (red) spectrograph. The gray regions show telluric contamination. Image credit: Mathias Zechmeister.

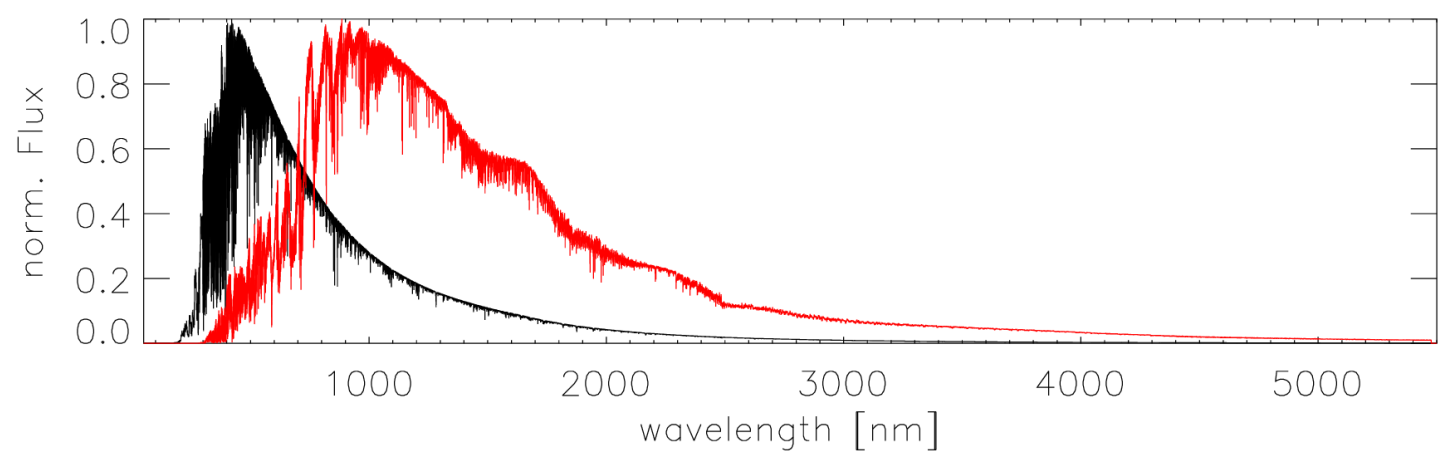

Figure 1.9.: Model spectrum energy distributions of a Sun-like star (G0 V, black) and an M-dwarf (M4.5 V, red), showing the peak being shifted towards longer wavelengths for lower temperature. 

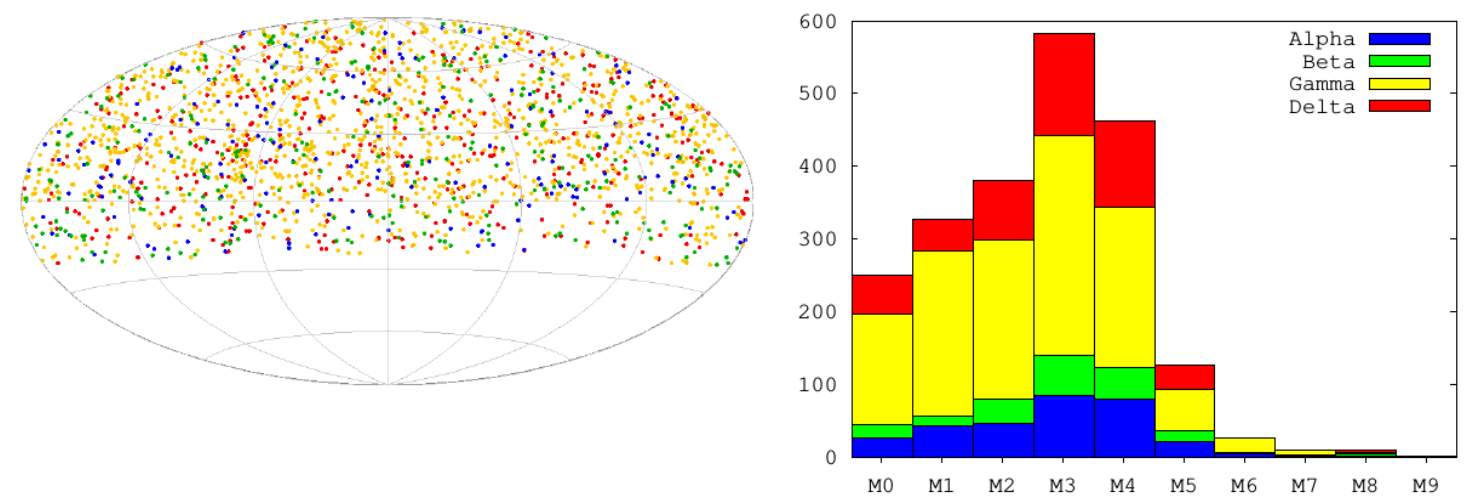

Figure 1.10.: Distribution of Carmencita stars on the sky (left) and as function of spectral type (right). The different priority groups are colour-coded. Image credit: CortesContreras (2016), reprinted with permission.

\subsubsection{Other M-dwarf surveys}

Besides CARMENES there are several other planet search surveys that focus mainly or partially on M dwarfs. A short description of some of them follows.

\section{Current}

HARPS HARPS (Mayor et al., 2003) is a fibre-fed, cross-dispersed echelle spectrograph with a resolution of $R \sim 115000$. The two fibres simultaneously observe the sky and a ThAr reference spectrum, covering a wavelength range from 380 to $690 \mathrm{~nm}$. The instrument is highly stable with a precision of $1 \mathrm{~ms}^{-1}$. Although the target stars show a wide range of spectral types, a sub-sample of $\mathrm{M}$ dwarfs has been observed by Bonfils et al. (2013).

MEarth Although MEarth (Irwin et al., 2015) is a transit survey in contrast to the other mentioned projects, it will monitor a huge sample of about 3000 nearby $\mathrm{M}$ dwarfs with the aim to detect Earth-sized planets in their habitable zone. It is operating with two stations in the northern and southern hemisphere. MEarth-North is located at the Fred Lawrence Whipple Observatory on Mount Hopkins, Arizona, and operating since September 2008. MEarth-South is located at the Cerro Tololo Inter-American Observatory in Chile, in operation since January 2014. Each station uses eight $0.4 \mathrm{~m}$ robotic telescopes. Due to the large spread of targets across the sky, a set of targets is observed in cyclic order during night and the telescopes return to each star every 20-30 minutes taking only a few exposures per visit. The data is analysed in real time, so an ongoing transit can be identified and follow-up observation can be performed immediately. 


\section{Future}

CRIRES+ CRIRES + (Follert et al., 2014) is an upgrade to the existing CRIRES instrument mounted at ESO VLT-UT1 on Paranal in Chile (Kaeufl et al., 2004). The highresolution spectrograph, covering only a short wavelength range of $15 \mathrm{~nm}$ in the nearinfrared, will be transformed into a cross-dispersed echelle spectrograph. It will also be equipped with a spectro-polarimetric unit and new gas-cells and etalons for wavelength calibration. The high spectral resolution of $R \sim 100000$ and the existing wavelength ranges will be preserved. CRIRES+ will focus on three scientific topics, the search for Super-Earths in the habitable zone of low-mass stars, their atmospheric characterisation via transit spectroscopy, and close investigation of stellar magnetic fields.

ESPRESSO The ESPRESSO (Echelle SPectrograph for Rocky Exo-planets Search and stable Spectroscopic Observations) instrument (Pepe et al., 2013) is a fiber-fed, cross-dispersed, high-resolution echelle spectrograph that will be mounted at the ESO VLT on Paranal in Chile. It will be able to collect light from all four UTs to observe very faint objects. The wavelength range from $380-780 \mathrm{~nm}$ will be covered with a spectral resolution of up to $R \sim 225000$. A long-term precision of around $10 \mathrm{cms}^{-1}$ allows for detection of Earth-mass planets around Sun-like stars. The target sample covers Sun-like and low-mass dwarfs down to spectral type M4 V.

HPF The Habitable Zone Planet finder (Mahadevan et al., 2015) will be mounted at the $10 \mathrm{~m}$ Hobby-Eberly telescope in Texas, USA to search for low-mass planets in the habitable zones of mid- to late-M dwarfs. The fibre-fed, high-resolution $(R \sim 50000)$ spectrographs cover the $\mathrm{z}, \mathrm{Y}$ and $\mathrm{J}$ bands in the NIR.

IRD The InfraRed Doppler instrument (Kotani et al., 2014) will be installed at the Subaru $8.2 \mathrm{~m}$ telescope to search for Earth-sized planets around M dwarfs. With high resolution of $R \sim 70000$ the spectral range spans from 0.97 to $1.75 \mu \mathrm{m}$. To achieve precisions of $1 \mathrm{~ms}^{-1}$ a laser frequency comb is used for wavelength calibration.

SPIRou SPIRou (Spectro-Polarimetre Infra-Rouge, Artigau et al., 2014) will be operational in 2017 at the Canada-France-Hawaii-Telescope. The high-resolution $(R \sim$ 70000 ) instrument covers a spectral range from 0.97 to $2.4 \mu \mathrm{m}$ in the NIR to hunt for Earth-sized planets orbiting mid-M dwarfs. The spectro-polarimeter will be used for the second science goal, which is the investigation of magnetic fields and their impact on planet formation. SPIRou will also serve as follow-up instrument for TESS (Transiting Exoplanet Survey Satellite) to determine the masses of planetary candidates via high-precision $\left(\sim 1 \mathrm{~ms}^{-1}\right)$ radial-velocity measurements. 


\subsection{Spectral characterisation}

In order to be able to characterise a planetary system it is important to accurately characterise the star. There are many properties (e.g. radius, mass, age, temperature, spectral type, surface gravity, metallicity, rotational velocity, colour, brightness, luminosity, ...) that can be determined using different techniques (e.g. interferometry, spectroscopy, photometry, eclipsing binaries, modelling, ...). However, from the stellar spectrum alone it is possible to derive many fundamental parameters, such as effective temperature, surface gravity, metallicity, spectral type and projected rotational velocity $(v \sin i)$. The importance of these parameters in the context of planet search is described shortly in the following.

Effective temperature The effective temperature, $T_{\text {eff }}$, of a star is a key parameter in stellar characterisation and refers to the temperature of a blackbody emitting the same amount of electromagnetic radiation as the star across all wavelengths. As mentioned in Section 1.1.1 the limits of the classical habitable zone are defined from the spectral type, equivalent to the temperature of the star, which is specified by the wavelength where the stellar energy distribution peaks. Furthermore this helps to calculate the amount of radiation the planet receives, and together with other planetary properties models can project, if the planet can be considered habitable. The key planetary properties are its mass and radius. The mass can be inferred from the radial-velocity shift if the mass of the star is known, e.g. from evolutionary models (Baraffe et al., 1998) or observational relations (Mann et al., 2015). Together with transit observations the degeneracy on $\sin i$ can be broken, and the planetary radius can be derived if the stellar radius is known, e.g. from interferometry or empirical relations using eclipsing binaries. Additionally, a transit gives the opportunity to characterise the planets atmosphere by doing transit spectroscopy.

Surface gravity Constraining the surface gravity, hereafter $\log g$, is relevant in two ways. From the spectral type alone it is not possible to unambiguously distinguish between a red dwarf and a giant star. Both energy distributions peak in the red to nearinfrared wavelength ranges and the spectra show absorption lines from molecules due to the low stellar temperature. Determination of $\log g$ can clarify the evolutionary state of the star. For main-sequence stars $\log g$ values lie between 4.5 and 5.5 (Angelov, 1996). Furthermore, the stellar mass and radius can be estimated with $\log g$ knowing or assuming the stellar density $\rho$.

Metallicity Several studies (e.g. Gonzalez, 1997, Gonzalez et al., 2001, Laws et al., 2003, Santos et al., 2004, Valenti \& Fischer, 2005) report a possible relation between stellar metallicity and giant planet occurrence for solar-like stars. Also for M dwarfs this trend seems to hold (e.g. Bonfils et al., 2007, Gaidos \& Mann, 2014, Neves et al., 2013, Schlaufman \& Laughlin, 2010), showing that planet hosting stars are slightly more metal-rich than stars without planets. These observations are consistent with the coreaccretion model explaining giant planet formation. In the accretion model (Ida \& Lin, 
2004, Wuchterl et al., 2000) dust agglomerates via inelastic collisions forming grains. As grains grow to larger bodies, gravitational accretion dominates the growth. For giant planets the core accretion phase needs to be sufficiently short in order to accumulate enough gas before dissipation. An increased metal content of the proto-planetary disk likewise increases the accretion rate since dust grains accumulate easier (Kornet et al., 2005, Mordasini et al., 2008). For lower mass planets the relation between higher metallicity and planet occurrence seems to flatten, with stars hosting a Neptune-sized planet having slightly lower metallicities compared to Jupiter host stars (Ghezzi et al., 2010. Mordasini et al., 2008, Sousa et al., 2008). However, the determination of metallicity can be important for selecting promising targets for planet host candidacy.

Rotational velocity Stellar rotation is one of many reasons for line broadening in the spectrum. The faster a star rotates the broader are the lines. This makes it more difficult to detect a radial-velocity shift. Because the radial-velocity method searches for a shift in the position of stellar spectral lines, the line centers need to be determined very accurately in order to calculate the wavelength shift. The sharper the lines are the more accurate is the calculation. Fast stellar rotation therefore reduces the accuracy and it might get very difficult or impossible to detect small shifts of a few $\mathrm{ms}^{-1}$. Additionally, it is known that there is a connection between rotation and activity. For Sun-like stars this was investigated by Noyes et al. (1984), Delfosse et al. (1998) and Pizzolato et al. (2003). M dwarfs have been studied by Mohanty \& Basri (2003), Reiners \& Basri (2007, 2010), Reiners et al. (2012) and Jeffers et al. (submitted). Jeffers et al. (submitted) provided the largest catalogue of around $750 \mathrm{M}$ dwarfs covering all spectral types from M0.0 V to M9.0 V. Their work is part of the science preparation for the CARMENES project. It was found that activity increases with increasing rotational velocity, up to a point where it saturates at a threshold velocity depending on the stellar mass (Pizzolato et al. 2003). Although the observed rotational velocity is only a projection and depends on the inclination angle $i$, it can be used as an indicator for high magnetic activity. Magnetic activity can induce additional signals in the radial-velocity curve and even mimic a planetary signal (e.g. Reiners et al., 2013, Robertson et al., 2014, Saar et al., 1998). Moreover, it affects the habitability of a potential planet, since stellar winds interact with the planet's atmosphere, leading to photo-evaporation of water and high UV-radiation that can damage DNA. However, the dimensions of this interaction depend on many parameters, such as activity level, distance between star and planet and strength of the planetary magnetosphere.

\subsubsection{Challenges with low-mass stars}

The determination of atmospheric parameters for low-mass stars is more difficult than for hotter Sun-like stars. The main opacity sources in cool stellar atmospheres are molecules instead of atoms. The molecules form forests of molecular lines; their formation is very complex and sometimes not well understood. For Sun-like stars Fischer \& Valenti (2005) determined stellar properties with high precision. For 1040 F-, G-, and K-type stars they obtained uncertainties of $44 \mathrm{~K}$ for $T_{\text {eff }}, 0.06$ dex for $\log g$, and 0.03 dex for metallicity. In 
contrast, for low-mass stars, accurate parameters, especially metallicity, are still difficult to achieve. Many works have focused on the determination of stellar parameters in lowmass stars. Van Belle \& von Braun (2009) and Boyajian et al. (2012) used the approach of measuring the effective temperatures of low-mass stars from photometrically determined bolometric fluxes and radii from interferometry. Other authors used theoretical models for parameter determination. Rojas-Ayala et al. (2012) observed spectra of 133 $\mathrm{M}$ dwarfs in the near-infrared K-band. They calculated the $\mathrm{H}_{2} \mathrm{O}-\mathrm{K} 2$ index, which quantifies the absorption due to $\mathrm{H}_{2} \mathrm{O}$ opacity. They calibrated the temperatures using BT-Settl models (Allard et al., 2011) for solar metallicity. Gaidos \& Mann (2014) investigated $121 \mathrm{M}$ dwarfs in the near-infrared K-band and visible wavelength range. For the K-band spectra they worked with spectral curvature indices to determine effective temperatures. For spectra in the visible wavelength range they used BT-Settl model atmospheres.

Several different approaches have been employed to measure the surface gravity. Ségransan et al. (2003) combined interferometric angular diameters and mass-luminosity relations to derive the stellar mass and from that the surface gravity. Del Burgo et al. (2013) and Rice et al. (2015) avoid these assumptions about mass and radius by directly determining the surface gravity from model fits. Maldonado et al. (2015) measured masses and radii of early $\mathrm{M}$ dwarfs from interferometry to derive the surface gravity.

On the other hand, it is far more difficult to accurately determine metallicities for lowmass stars. A usual "line-by-line" approach, as used for Sun-like stars, cannot be employed, because of the vast amount of molecular lines and bands. A more complex method is necessary, like a full spectral synthesis. A detailed review about different approach and methods was presented by Neves et al. (2012) and Passegger et al. (2016). Independent measurements of M-dwarf metallicities are possible if the star is part of an FGK $+\mathrm{M}$ binary system. As mentioned before, metallicity determination in Sunlike stars is less complex. Since binary systems are believed to have formed out of the same molecular cloud, both components are expected to have the same metal abundance. Bonfils et al. (2005) investigated $20 \mathrm{FGK}+\mathrm{M}$ binaries and presented a colour-metallicity relation for $\mathrm{M}$ dwarfs. This makes it possible to derive metallicities directly from the colour and reduces the complexity of the problem. Rojas-Ayala et al. (2012) measured the equivalent width of $\mathrm{NaI}$ and $\mathrm{CaI}$ in the near-infrared and calibrated their metallicities with $18 \mathrm{FGK}+\mathrm{M}$ binaries. They discovered that the relation found by Bonfils et al. (2005) underestimates metallicities for stars with solar and super-solar metallicities. The metallicities determined by Rojas-Ayala et al. (2012) also agree with those from

Gaidos \& Mann (2014), who obtained them from relations between metallicity and the line strength of atomic lines in the near-infrared H- and K-bands. Maldonado et al. (2015) measured metallicities and effective temperatures from optical spectra pseudo equivalent widths.

The PHOENIX model atmospheres, developed by Hauschildt et al. (1999) and Allard et al. (2001), have been widely used in the past decade. Jones et al.(1996) first used the models to perform a line-by-line analysis of atomic lines in the near-infrared, whereas Gizis (1997) was matching optical spectra with low resolution to these models. Highresolution spectra have then been used by Valenti et al. (1998) and Zboril \& Byrne (1998). However, the results of these studies illustrate the problems with metallicity determination in M dwarfs. For GJ 699 (Barnard's star), Jones et al. (1996) found [Fe/H]=- 
0.75, whereas Rojas-Ayala et al. (2012) reported $[\mathrm{Fe} / \mathrm{H}]=-0.39 \pm 0.17$ and Passegger et al. (2016) determined $[\mathrm{Fe} / \mathrm{H}]=-0.13 \pm 0.11$. Rajpurohit et al. (2013) observed $152 \mathrm{M}$ dwarfs and obtained low- and medium-resolution spectra. They determined effective temperatures using BT-Settl models and a $\chi^{2}$-method. Rajpurohit et al. (2014) presented an improvement of this work, by determining surface gravity and metallicity from highresolution spectra of $21 \mathrm{M}$ dwarfs. Similar to my work and the work of Passegger et al. (2016), they interpolated the model grid points to get accurate parameters. Mann et al. (2015) determined effective temperatures from fitting BT-Settl models to optical spectra, and metallicities using the empirical relation stated by Mann et al. (2013a, 2014) and equivalent widths of near-infrared atomic lines. Passegger et al. (2016) obtained effective temperatures, surface gravities and metallicities from fitting the latest version of PHOENIX-ACES model atmospheres to high-resolution optical spectra. Their results for $T_{\text {eff }}$ and $\log g$ agree well with results from Mann et al. (2015), Gaidos \& Mann (2014) and Rojas-Ayala et al. (2012). For metallicity they found an average offset of +0.31 dex compared to literature values. However, they also investigated the M-dwarf Gl 667C, which is part of an FGK $+\mathrm{M}$ binary system with a known metallicity. The metallicity determined for the M-dwarf agrees with the metallicity of the higher mass companion, which supports their method. In this work I improved the method developed by Passegger et al. (2016) and applied it to a big sample of M dwarfs. In the following chapter I will describe the method in detail. 



\section{Methods}

In this chapter I will present the method I used for determining the stellar parameters of low-mass stars. This includes a short description of the PHOENIX-ACES model atmospheres, followed by the presentation of the algorithm as well as the different spectral ranges and fitting regions in the visible and near-infrared.

\subsection{The PHOENIX-ACES models}

The PHOENIX-ACES grid is described in detail by Husser et al. (2013). Table 2.1 summarises the parameters of the grid. For all models with temperatures $T_{\text {eff }} \leq 12000 \mathrm{~K}$ local thermodynamic equilibrium (LTE) has been assumed. For the wavelength range I use for fitting, i.e. $3000-25000 \AA$, the sampling of the grid corresponds to a resolution of $\mathrm{R}=500000$. Alpha element abundances of $[\alpha / \mathrm{Fe}] \neq 0$ are only available for $T_{\text {eff }}$ between $3500 \mathrm{~K}$ and $8000 \mathrm{~K}$ and for sub-solar metallicities from -3 to 0 dex. Dust settling was ignored in the models, since all of them have $T_{\text {eff }} \geq 2300 \mathrm{~K}$. The latest version of solar abundances was used, published by Asplund et al. (2009). The models use a new equation of state, the Astrophysical Chemical Equilibrium Solver (ACES), which extended the validity range in gas pressure and temperature compared to previous equations of state. Husser et al. (2013) implemented a new parametrisation of the micro-turbulence and mixing-length, which gave rise to some significant differences between the new and previous PHOENIX models, especially concerning different line and molecular band strengths, such as the $\epsilon$ - and $\gamma$-TiO bands. The mixing length theory is used to describe stellar convection. The micro-turbulence influences the line opacity and therefore the line strength and has to be included during the line formation process. Macro-turbulence does not affect the line strength, but triggers micro turbulent motions and is related via $v_{\text {micro }}=0.5 \cdot\left\langle v_{\text {conv }}\right\rangle .\left\langle v_{\text {conv }}\right\rangle$ represents the mean value of the macro-turbulence and is provided by the PHOENIX model atmospheres by averaging all non-zero convective velocities of all atmospheric layers. For cool main-sequence stars, Husser et al. (2013) showed that the micro-turbulence is smaller than $1.5 \mathrm{kms}^{-1} 1$ for solar abundances. 
Table 2.1.: Parameter space of the PHOENIX-ACES grid. Credit: Husser et al. (2013), Table 1, reproduced with permission, (CESO.

\begin{tabular}{ccc}
\hline \hline Variable & Range & Step size \\
\hline$T_{\text {eff }}$ & $2300-7000$ & 100 \\
& $7000-12000$ & 200 \\
$\log g$ & $0.0-+6.0$ & 0.5 \\
{$[\mathrm{Fe} / \mathrm{H}]$} & $-4.0--2.0$ & 1.0 \\
& $-2.0-+1.0$ & 0.5 \\
{$[\alpha / \mathrm{Fe}]$} & $-0.2-+1.2$ & 0.2 \\
\hline
\end{tabular}

\subsection{The fiasco-code}

The fiasco-code was developed to fit the above mentioned PHOENIX-ACES models to observed spectroscopic data and to find the model with the best fitting parameters using a $\chi^{2}$-method. The code is fully automated. It reads in a list of spectra that should be processed and returns best fit parameters and plots in an output file. In the following subsections the functionality of the code will be explained in detail.

\subsubsection{The fitting procedure}

The code for parameter determination consists of two parts. The first part uses the grid of model spectra described in Section 2.1 in a wide range around the expected parameters of the star. Firstly, to match the instrumental resolution, the model spectra are convolved with a Gaussian. Then the average flux of the models and the observed spectrum is normalised to unity. Since M dwarfs show a vast amount of atomic and molecular lines in their spectra, it is not possible to define a continuum similar to Sun-like stars. Instead, a pseudo-continuum is defined by a large number of points between the spectral lines. A polynomial of a given degree (default is three, one is used here) is fitted along these points using a least-squares fit. Then the continuum points that deviate from the current fit less than three rms are selected by applying an iterative polynomial fit. Typically ten iterations are sufficient to obtain a polynomial that provides a good estimate for the pseudo-continuum. Fig. 2.1 illustrates the procedure for different iteration steps in one wavelength range.

Moreover, the model wavelength grid is interpolated to match the wavelength sampling of the observed spectrum. Due to the spatial radial velocity of the star, the observed spectrum is shifted with respect to the rest frame. This shift is determined using a cross correlation with a default model spectrum. The observed spectrum is then shifted according to the measured radial velocity shift in each wavelength range.

Then each wavelength point of each model spectrum is compared to the stellar spectrum. The $\chi^{2}$ is calculated to find a rough global minimum for different wavelength ranges in the visible or near-infrared regime. The parameters for the three best minima are given as output because it is possible that the real global minimum lies between the model grid points. Additionally, the algorithm gives $\chi^{2}$-maps for different combinations of all 
output parameters and $\chi^{2}$-curves for each parameter, and a plot of the spectrum and the best fit model in each wavelength range. This can be seen in Figs. 2.2 and 2.3 .

In the second part, the region around the global minimum found in the first part is explored on a finer grid. Exploring a finer grid involves interpolation between the model grid points. I investigated linear and quadratic interpolations on a small sample of stars and concluded that there is no significant difference between the resulting parameters. To save computation time, a linear interpolation is used. A downhill simplex then walks around in the parameter space. For every set of parameters, a function looks for the nearest model grid points and interpolates the corresponding model spectra. To remove false signals from cosmics or bad pixels, which appear as strong peaks in the spectrum, a simple sigma clipping procedure is performed before the algorithm continues. To account for the rotational velocity of the star the model spectrum is broadened using a broadening function. The function calculates the effect on the line spread function caused by the rotation of the star. It assumes a constant limb darkening coefficient of 0.6 (typically valid for $\mathrm{M}$ dwarfs) across the stellar disk. The resulting line spread function is convolved with the model spectrum. The input values for the rotational velocity will be discussed later. Different fitting algorithms have been investigated to analyse the grid in more detail and fit models to the observed spectrum. This was done by calculating a detailed $\chi^{2}$-map for 50 stars and identifying the global minimum on this map. I used four different fitting routines, namely the IDL built-in functions curvefit and MPFIT, as well as AMOEBA and the downhill simplex, to find the global minimum. The results show that the downhill simplex finds the global minimum more precisely than the other algorithms, which also tend to get stuck in local minima more easily. Since the $\chi^{2}$-maps can feature plateaus the step size of the curvefit and MPFIT procedures can be too small to escape. The AMOEBA routine gives good results as well, but the downhill simplex shows better stability when it comes to large sample sizes. An example for the performance of the four algorithms is shown in Fig. 2.4. Back to the algorithm, the $\chi^{2}$ is calculated for the interpolated spectrum and the observed spectrum, and the value is returned to the simplex, which decides in which direction in the parameter space to move next or if the best $\chi^{2}$ is already found. The whole procedure is done for all three minima found in the first part. The result is a plot of the observed spectrum with the best fit model. Fig. 2.5 shows an example of a typical spectrum of an M0.5-star observed with CARMENES with the best fit model. Fitted lines are identified and marked. 

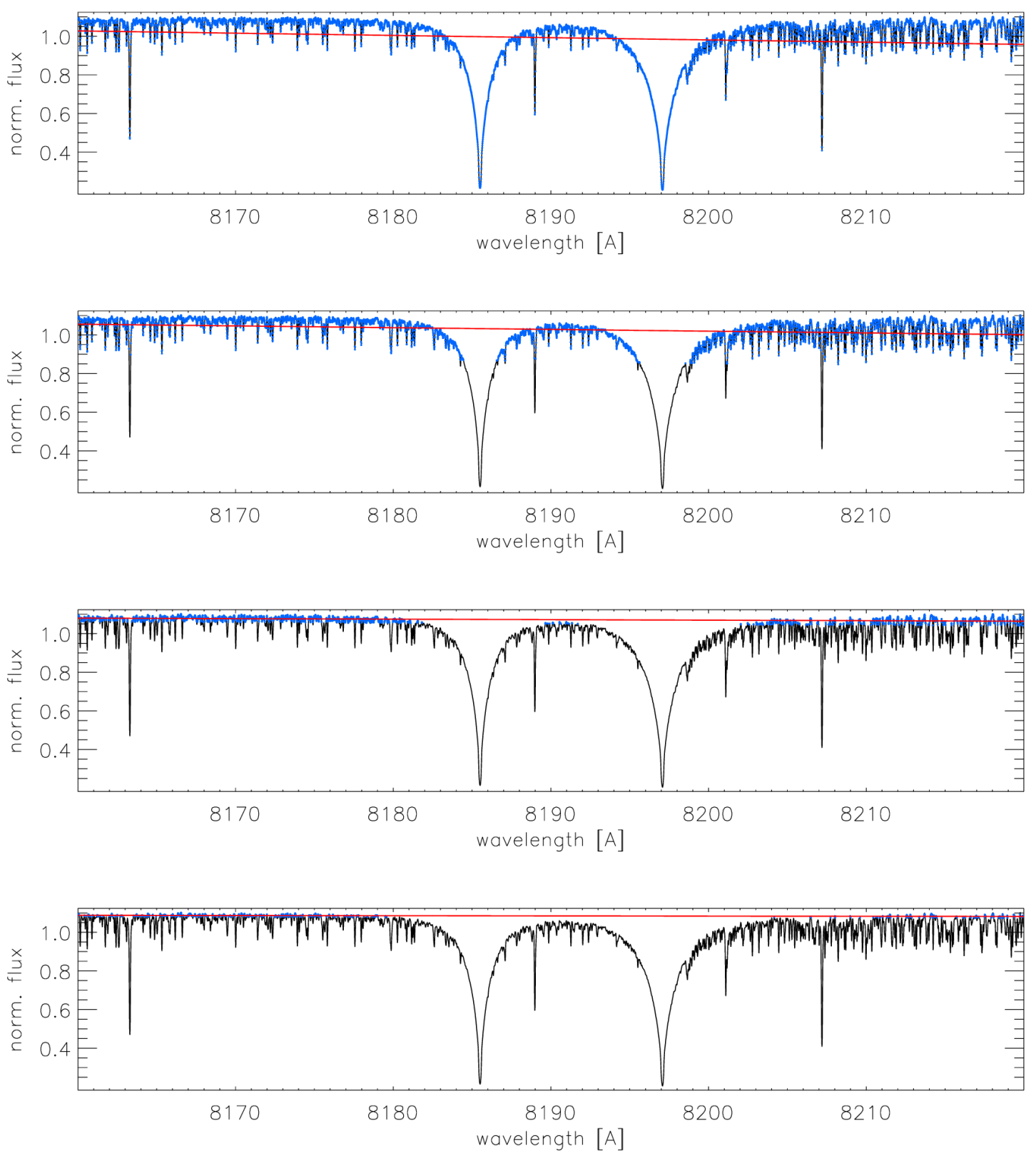

Figure 2.1.: Illustration of continuum fitting procedure for different iteration steps. Black shows a model spectrum for a typical M3-star in the region of the Na-lines. The blue dots mark all points that deviate by $3 \mathrm{rms}$ from the mean, the continuum (red) is fitted through these points and the deviation of $3 \mathrm{rms}$ is calculated again. The panels represent the fit after iteration $0,1,5$ and 10 (from top to bottom). 

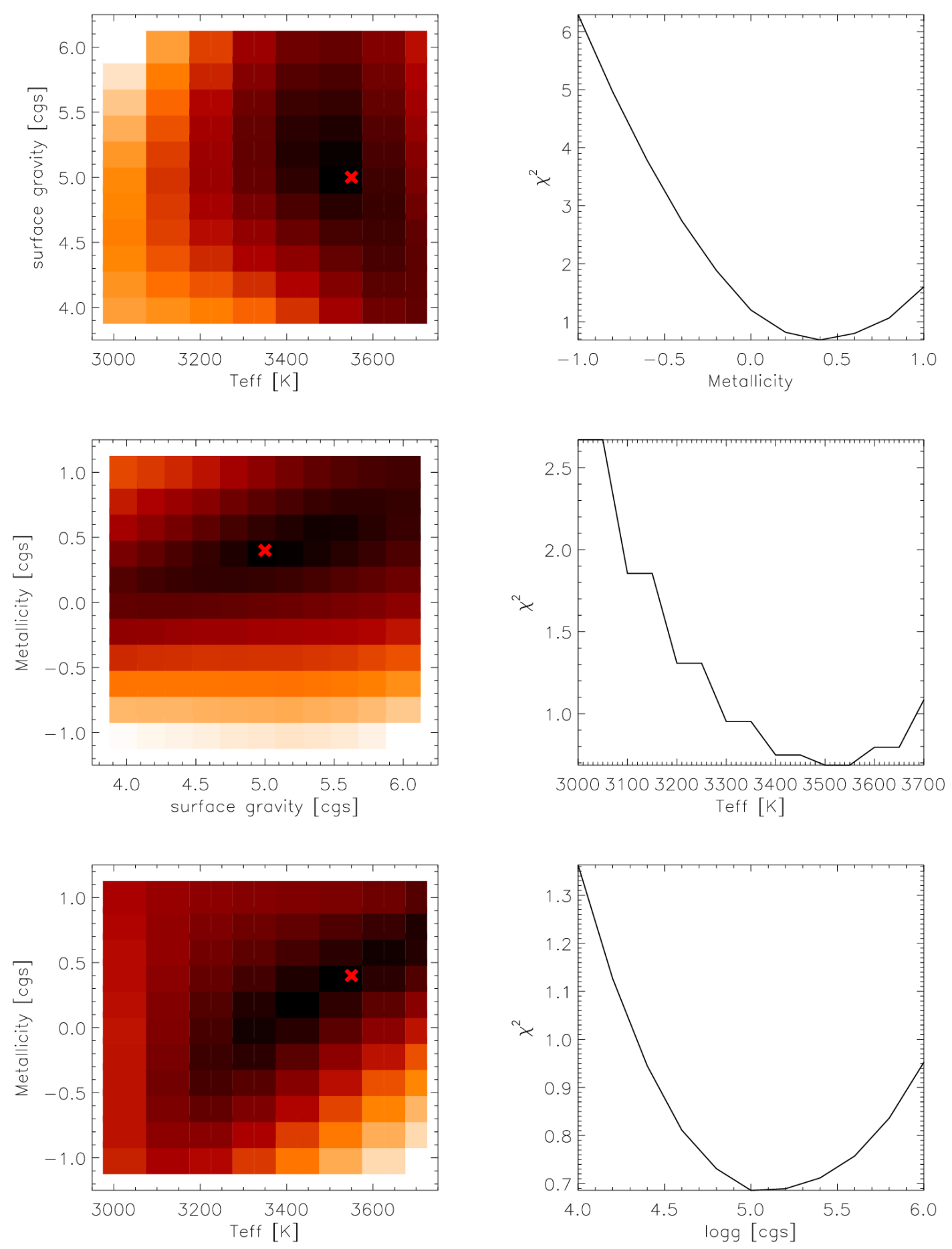

Figure 2.2.: Results from the first part of the fitting procedure on the coarse grid. Left column: $\chi^{2}$-maps for all parameter combinations. The red cross marks the minimum. Right column: $\chi^{2}$-curves for each parameter. This kind of plot is produced for all three minima. 

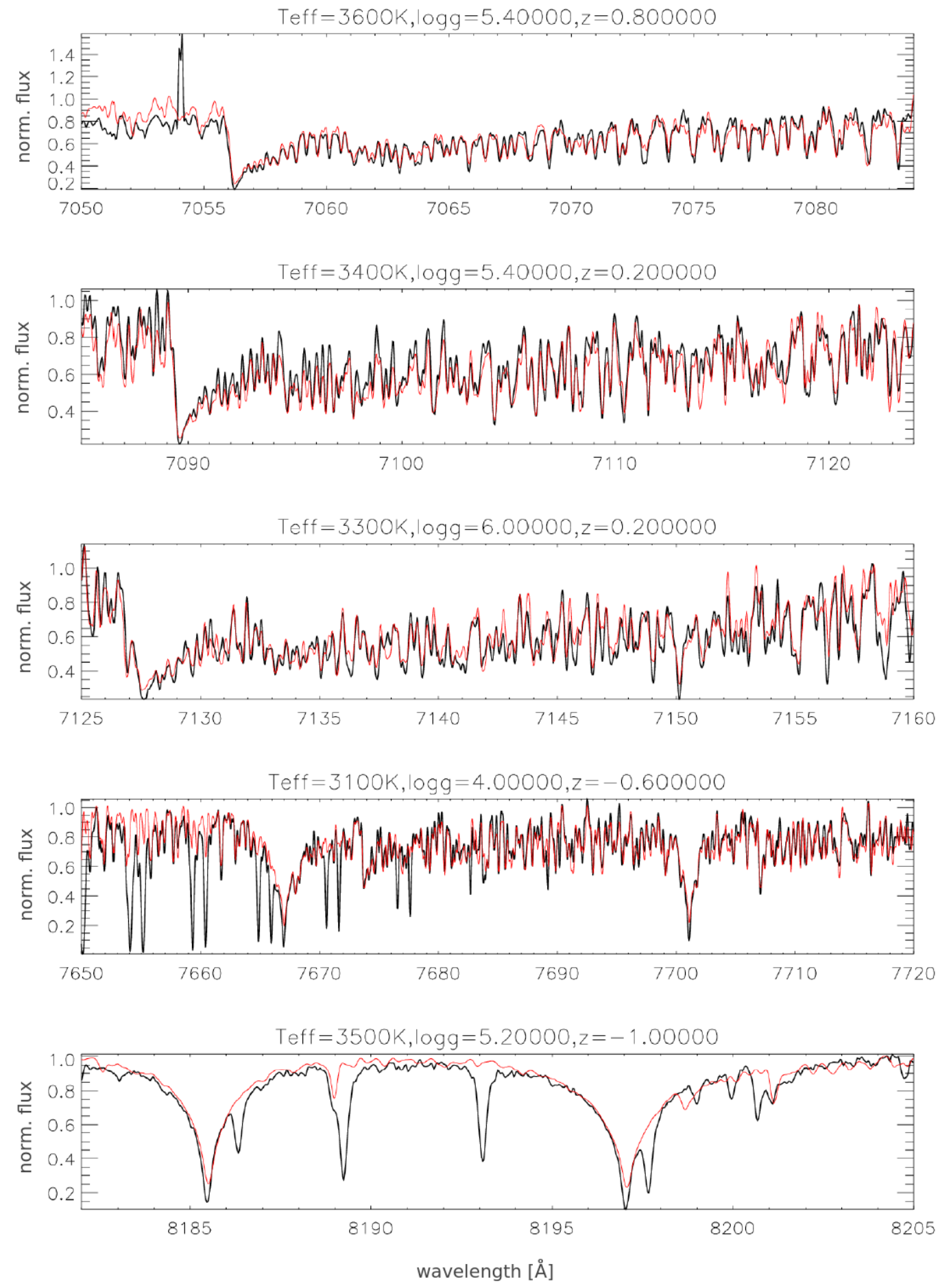

Figure 2.3.: Best model fit (red) to the observed spectrum (black) for different wavelength region between 7050 and $8205 \AA$. The best fit parameters are written on the top of each panel. This kind of plot is produced for all three minima from the first part of the fiasco-code. 

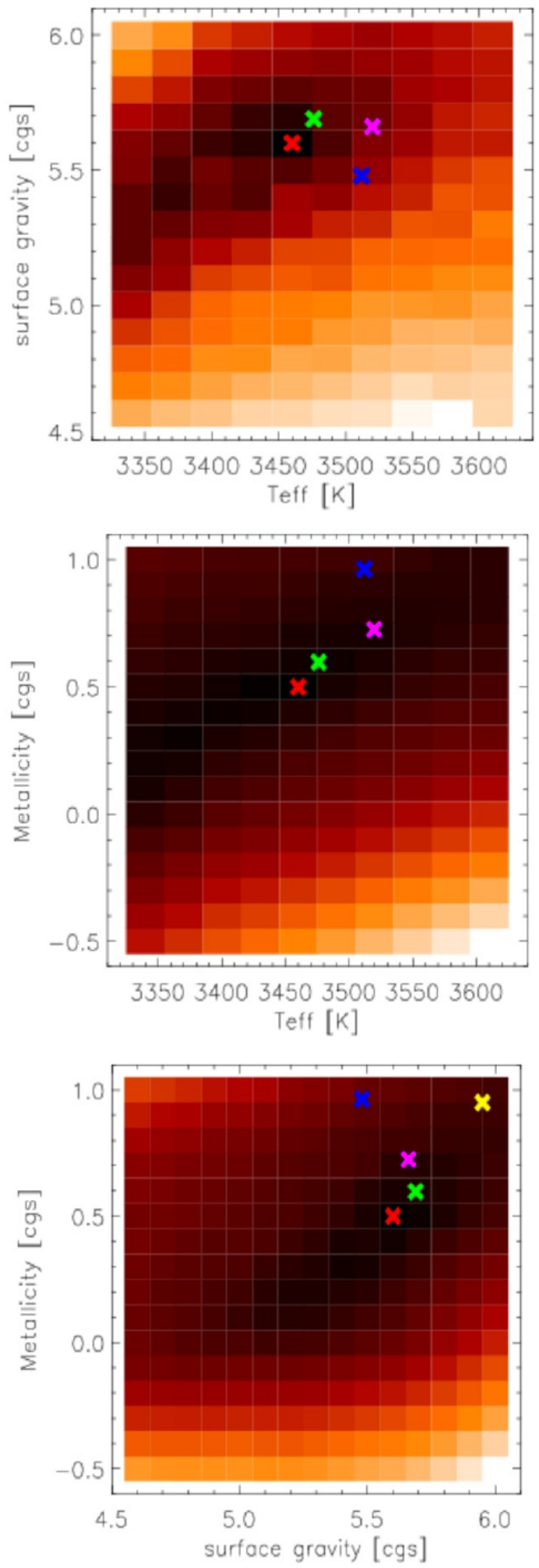

Figure 2.4.: $\chi^{2}$-maps for different combinations of parameters from the fit-algorithm test. The global minimum of each map is marked with a red cross. The results of the fitting algorithms are marked in other colours: downhill simplex (green), AMOEBA (purple), curvefit (blue), and MPFIT (yellow, not shown in the top and middle panel, because the result is out of range). 

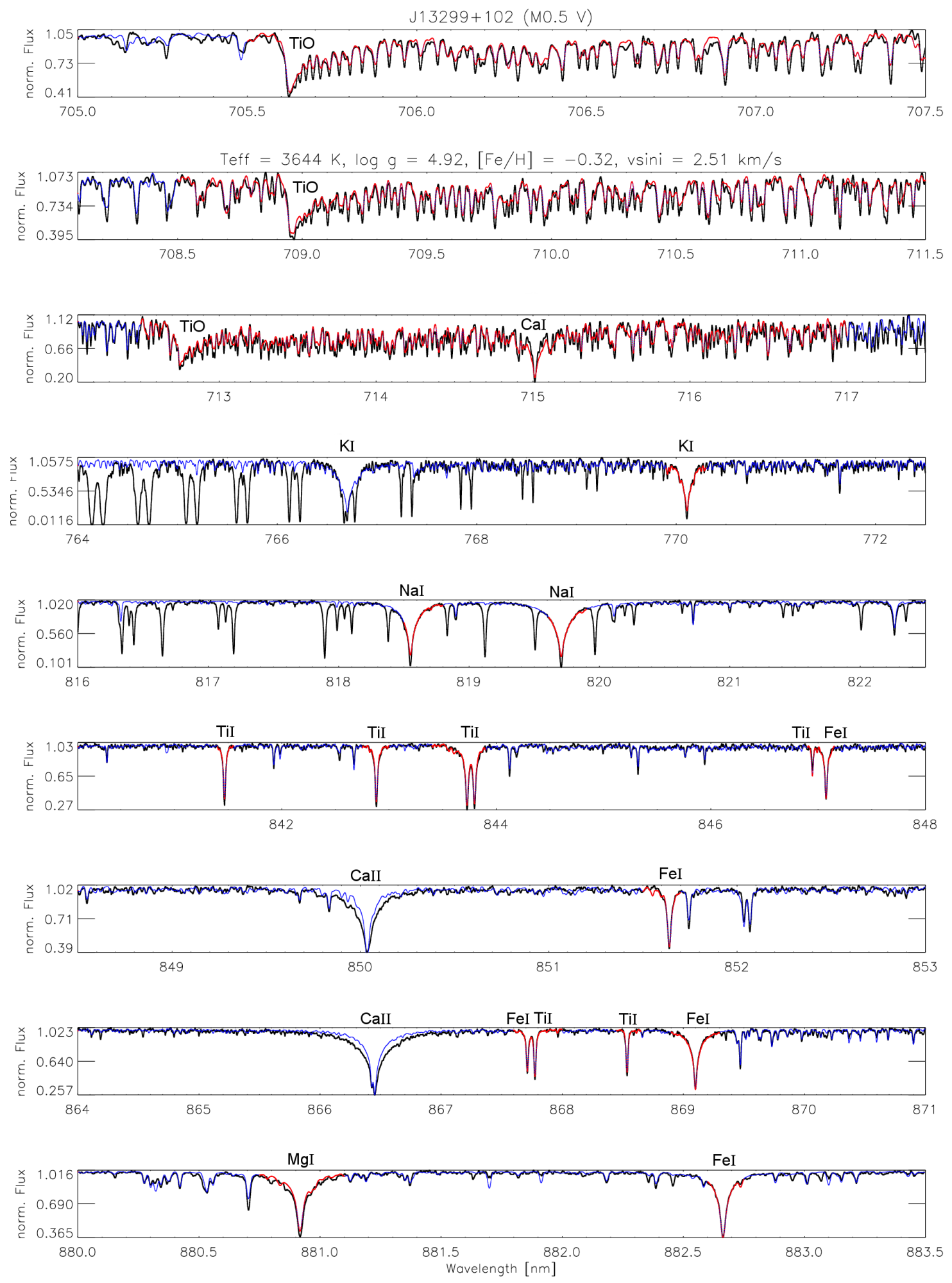

Figure 2.5.: Final result showing a typical spectrum of an M0.5 V star: GJ 514 (black) observed with CARMENES together with the best-fit model (blue). The red lines mark the regions and lines used for $\chi^{2}$-minimisation, best fit parameters can be found on top of the second panel. 


\subsubsection{Accounting for $v \sin i$}

As mentioned above the stellar rotational velocity, $v \sin i$, is incorporated in the code. In an early version $v \sin i$ was treated as a free parameter. The downhill simplex investigated a 4-D parameter space, and after each iteration the interpolated model was broadened according to the selected $v \sin i$. A comparison with literature values for $T_{\text {eff }}, \log g$ and metallicity showed that the calculated parameter values tend to be higher. Jeffers et al. (submitted) determined $v \sin i$ s from the same sample of CAFE, FEROS and HRS observations using cross-correlation, which allowed me to fix $v \sin i$ for each star to decrease the number of free parameters. It not only reduces the computation time, but also makes the method more stable. Most stars have $v \sin i$ values less than $3 \mathrm{kms}^{-1}$, which is the lower detection limit for resolutions similar to CARMENES, for which $v \sin i$ can be reliably measured. For some stars Jeffers et al. (submitted) provides $v \sin i$ s from other sources. These sources are given below Table A.1 in the Appendix. For 99 stars there are no $v \sin i$ s available, mostly due to binarity or poor quality spectra. In these cases I assumed a default value of $3 \mathrm{kms}^{-1}$. Stars without $v \sin i$ measurements, for which I obtain good fits, are included in Table A.2. in the Appendix for completeness, but not in the plots in Section 4.

\subsubsection{Weighting}

After updating the algorithm to keep $v \sin i$ fixed, the results were in better agreement with literature. Still, there was a trend towards higher $T_{\text {eff }}(\sim 200 \mathrm{~K}$ hotter $), \log g$ ( $\sim 0.5$ dex higher, up to $5.8 \mathrm{dex})$ and metallicity ( $\sim 0.5 \mathrm{dex}$ higher, up to $0.8 \mathrm{dex})$. From inspection of the final fits I could see that the algorithm was sometimes having problems to properly fit single lines. It concerned the depths as well as the wings of certain lines, which caused $\log g$ and metallicity to be too low or too high.

A way to overcome this is to change the weighting for some lines during the $\chi^{2}$ calculation. First, in order so that all lines contribute to the $\chi^{2}$ in the same way, the fitting regions were weighted depending on the number of points within the region. This prevents some regions from contributing more to the $\chi^{2}$ because they contain a higher number of points. It also allows for higher or less weighting of certain regions. The lines of $\mathrm{K} \mathrm{I}$ ( $\lambda 7701 \AA)$, Na I ( $\lambda 8185.5$ and $8197 \AA$ ) and Mg I ( $\lambda 8809 \AA)$ were weighted twice as much as the other fitting regions because these lines are especially sensitive to $\log g$ and metallicity, and therefore help to constrain the fit to certain reasonable values.

\subsubsection{Determination of $\log g$}

In a detailed analysis of the preliminary results I identified a degeneracy between $T_{\text {eff }}$, $\log g$ and $[\mathrm{Fe} / \mathrm{H}]$. Fig. 2.6 shows $\chi^{2}$-maps for different parameter planes. For Gl 908 a largely extended minimum is clearly visible in the $T_{\text {eff }}-[\mathrm{Fe} / \mathrm{H}]$ map, also a trend towards very low $\log g$ and $[\mathrm{Fe} / \mathrm{H}]$ values can be seen. A way to break this degeneracy is to determine $\log g$ with an independent method.

I decided to use evolutionary models from Baraffe et al. (1998), who provide several stellar parameters including effective temperature and $\log g$ for metallicities 0.0 and 

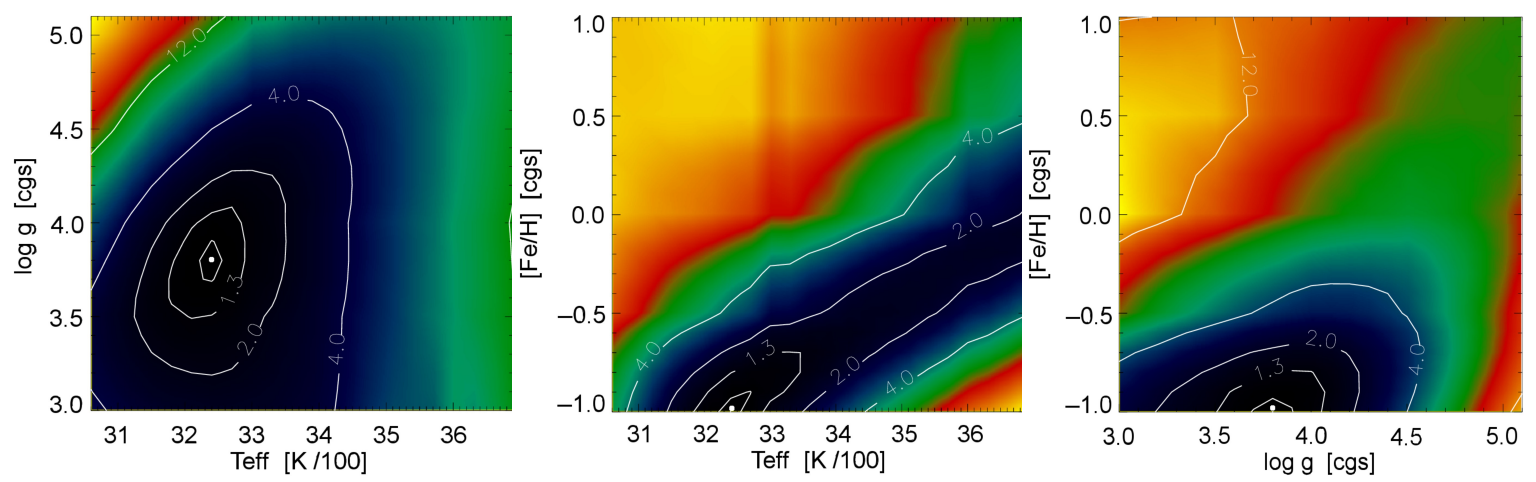

Figure 2.6.: $\chi^{2}$-maps for G1 908 for different combinations of stellar parameters. A large extended minimum is visible in the middle panel showing the degeneracy between $T_{\text {eff }}$ and $[\mathrm{Fe} / \mathrm{H}]$. The right panel illustrates very low $\log g$ and $[\mathrm{Fe} / \mathrm{H}]$ values.

-0.5 dex for different stellar ages. I assumed a stellar age of 5 Gyr for all target stars, since the $T_{\mathrm{eff}}-\log g$ relations for ages between 1 and 5 Gyr agree with each other up to a temperature of $4000 \mathrm{~K}$. Fixing $\log g$ reduced the number of free parameters in the algorithm to two. Based on $T_{\text {eff }}$ and $[\mathrm{Fe} / \mathrm{H}]$ chosen by the downhill simplex, $\log g$ is determined from the relations. I linearly interpolated the relations for metallicities between 0.0 and -0.5 dex, and extrapolated for metallicities smaller than -0.5 dex and larger than 0.0 dex. The differences in $\log g$ amount only 0.1 for metallicities between 0.0 and -0.5 dex. Therefore the error from the inter- and extrapolation is much smaller than the error from fitting (see next subsection) and therefore can be neglected. Baraffe et al. (2015) published new evolutionary models, but these do not show any difference from the Baraffe et al. (1998) models for the temperature range of M dwarfs up to $4000 \mathrm{~K}$.

\subsubsection{Error estimation}

In this framework the error for each parameter cannot be calculated directly from $\chi^{2}$ because the errors are not statistical, but mainly dominated by systematics. A statistical error determined from $\chi^{2}$ is only of the order of a few Kelvin in temperature. To estimate the error originating from fitting, a set of 1400 model spectra was produced. Their parameters $T_{\text {eff }}, \log g$ and $[\mathrm{Fe} / \mathrm{H}]$ were randomly and uniformly distributed. I also account for the different spectral resolutions of the spectrographs and wavelength ranges used for observations. To simulate a signal-to-noise ratio of about 100, Poisson noise was added to each spectrum. Then I used the algorithm described above to determine parameters. The standard deviations of the differences between input and output parameters are defined as errors. The results show that for the different resolution of the CAFE (62 000), FEROS (48 000), HRS (60 000) and CARMENES (82 000) spectrographs the errors differ by only a few $\mathrm{K}$ in $T_{\text {eff }}$ and less than 0.05 dex in $\log g$ and metallicity. For this reason I assume an average error for each parameter for all these spectrographs. The same applies to the X-Shooter spectra $(R \sim 5400-18000)$ for each wavelength range. All errors are presented in Table 2.2. For simplicity I will use the acronym $C F H$ standing for CAFE, FEROS and HRS. 
In general, the magnitude of the errors is influenced by the resolution and quality of the spectra, the method used for deriving parameters and the selection of lines. The errors for the $\mathrm{CFH}$ and CARMENES spectra seem to be quite fair for $\mathrm{M}$ dwarf spectra. In $\log g$ and metallicity the errors might be a bit large, which could be related to the way of determining $\log g$ and the selection and weighting of used lines. An issue I found with the latter will be discussed in more detail in Section 5.2. For X-Shooter the errors in VIS are much larger because of the lower resolution of the spectra compared to $\mathrm{CFH}$ and CARMENES. In the NIR the treatment of tellurics and parameter sensitive lines contribute a great part to the error. It is clear from the errors and the results in Section 4 that a detailed analysis of the NIR data needs a more careful handling of these elements, which will be done in a later stage of this project. For the TripleSpec spectra the large difference between the errors in the different bands is worth a notice. In the $\mathrm{H}$-band the errors are quite small, only a few $\mathrm{K}$ in temperature, whereas in the $\mathrm{K}$-band the errors are much larger. The H-band range between 1570 and $1595 \mathrm{~nm}$ seems to be very sensitive to temperature; a change of a few 10s of Kelvin has a large effect on the energy distribution. In the K-band, on the other hand, the energy distribution is rather stable within 150-200 K, which explains the larger error.

Table 2.2.: Systematic errors from fitting for stellar parameters

\begin{tabular}{lcccc}
\hline \hline Spectrograph & $\lambda$-range & $T_{\text {eff }}[\mathrm{K}]$ & $\log g[\mathrm{cgs}]$ & {$[\mathrm{Fe} / \mathrm{H}]$} \\
\hline CFH \& CARMENES & VIS & 93 & 0.29 & 0.25 \\
TripleSpec & J & 94 & 0.33 & 0.34 \\
& H & 18 & 0.33 & 0.19 \\
& K & 179 & 0.32 & 0.38 \\
X-Shooter & VIS & 121 & 0.32 & 0.33 \\
& J & 107 & 0.32 & 0.25 \\
& H & 57 & 0.33 & 0.21 \\
\hline
\end{tabular}

\subsection{Spectral regions}

\subsubsection{The visible regime}

Because the parameters effective temperature, surface gravity and metallicity can be strongly degenerated in cool stars, I chose several spectral regions which are sensitive to one or more stellar parameters simultaneously. Passegger et al. (2016) studied the dependencies of spectral lines on the stellar parameters $T_{\text {eff }}, \log g$ and $[\mathrm{Fe} / \mathrm{H}]$. The molecular TiO-bands around $7050 \AA$ and $8430 \AA$ ( $\gamma$-and $\epsilon$-electronic transitions, respectively) are very dependent on $T_{\text {eff }}$, but almost insensitive to $\log g$. Fig. 2.7 shows a $\chi^{2}$-map of the $\mathrm{TiO}$ and alkali lines in the $T_{\text {eff }}-\log g$ plane. The alkali lines are more sensitive to $\log g$. The dependence of increasing line widths towards lower $\log g$ values is caused by the increasing micro-turbulent velocities in cooler stars. The $\mathrm{K}$ - and Na-line pairs at around $7680 \AA$ and $8190 \AA$, respectively, show large changes in their line wings because of 
pressure broadening. For metallicity determination I use all the mentioned regions, since all of them are highly sensitive, as shown in Fig. 2.8. The alkali lines were also used by Rajpurohit et al. (2014) for $\log g$ and metallicity determination, as well as several TiObands because of their temperature sensitivity. Additionally, I include several Ti I and Fe I lines between $8410 \AA$ and $8835 \AA$. As mentioned in Passegger et al. (2016), the Kand Na-line pairs as well as the TiO bands are contaminated by telluric lines from $\mathrm{O}_{2}$ and $\mathrm{H}_{2} \mathrm{O}$. Due to the strong contamination around the first $\mathrm{K}$ I line $(\lambda 7665.5 \AA)$, I decided to omit this line in the improvement stage of the code. Fig. 2.9 shows a plot of the spectrum of HD 285968, observed with FEROS, together with modelled telluric spectra of $\mathrm{O}_{2}$ and $\mathrm{H}_{2} \mathrm{O}$ (Husser \& Ulbrich, 2014). Other lines from atmospheric molecules, like $\mathrm{O}_{3}, \mathrm{CH}_{4}$ or $\mathrm{CO}_{2}$ showed no influence in the specified wavelength regions and are therefore not plotted. For the other lines I use masks to exclude the telluric lines from the fit.
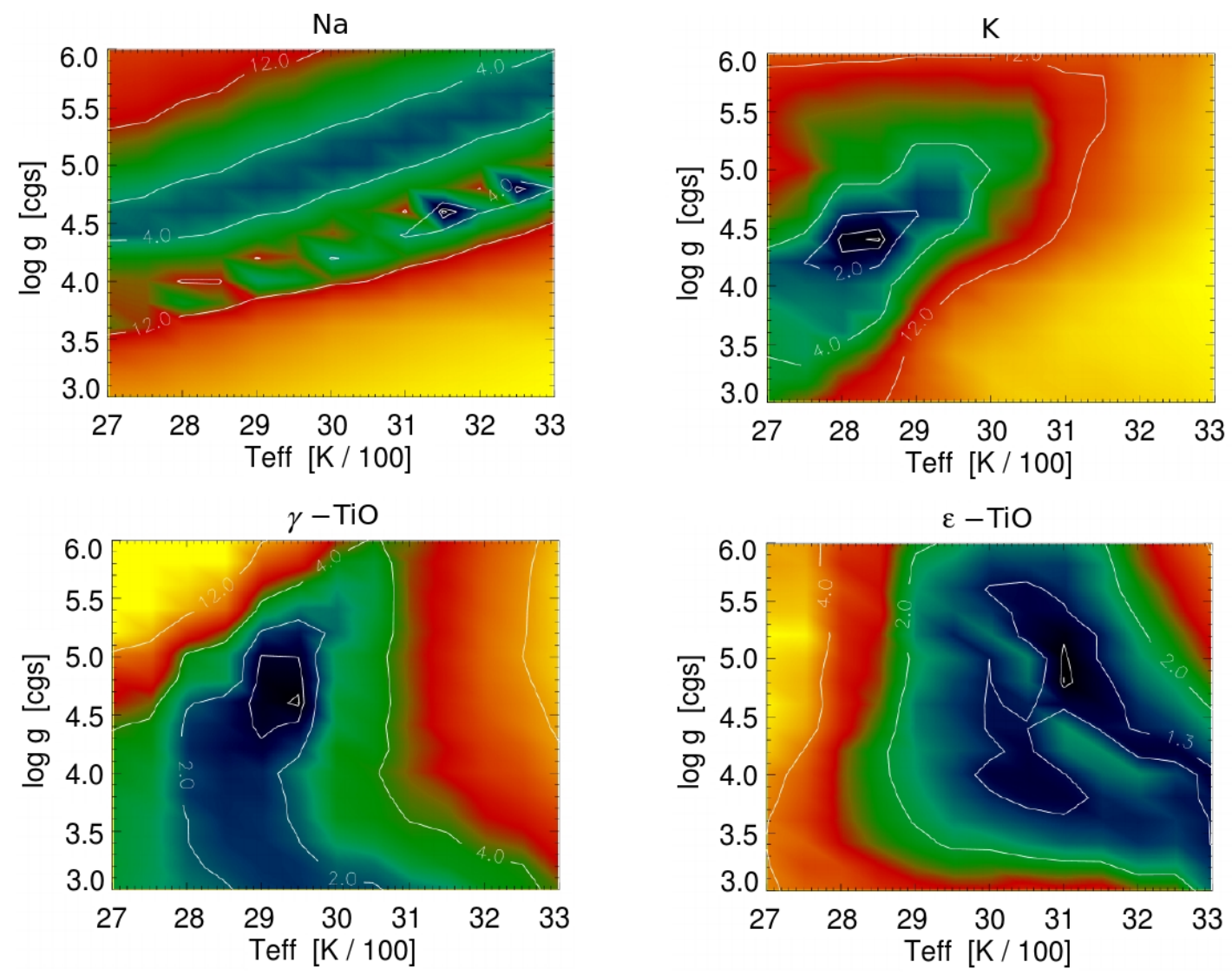

Figure 2.7.: $\chi^{2}$-maps in the $T_{\text {eff }}-\log g$ plane of the used TiO bands and alkali lines for the M dwarf GJ 551 with $\log g=5.02, T_{\text {eff }}=2927 \mathrm{~K},[\mathrm{Fe} / \mathrm{H}]=-0.07$, and SNR $\sim 100$. Image credit: Passegger et al. (2016), Fig.1, reproduced with permission, (c)ESO. 

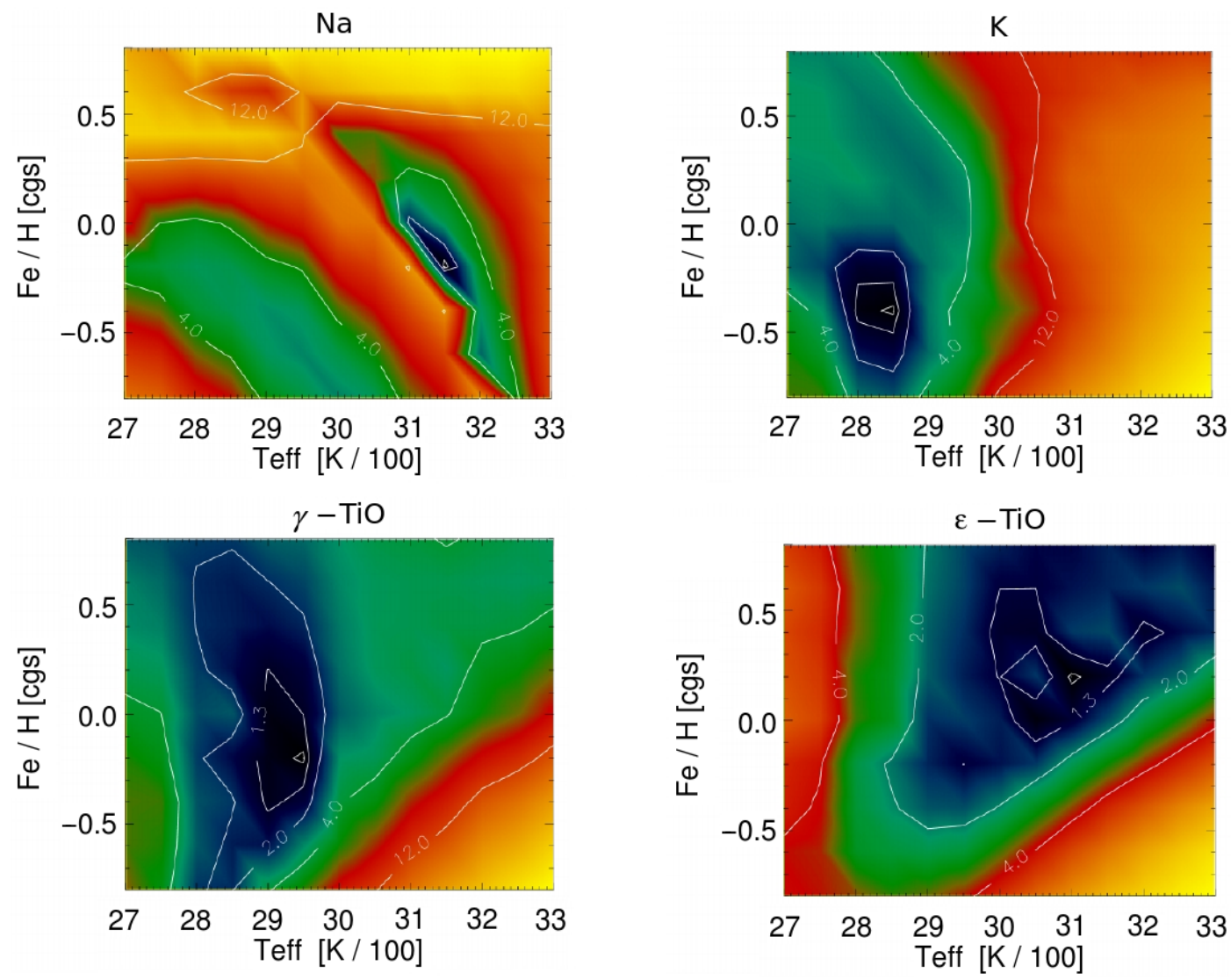

Figure 2.8.: $\chi^{2}$-maps in the $T_{\text {eff }}-[\mathrm{Fe} / \mathrm{H}]$ plane of the used TiO bands and alkali lines for the M dwarf GJ551 with $\log g=5.02, T_{\text {eff }}=2927 \mathrm{~K},[\mathrm{Fe} / \mathrm{H}]=-0.07$, and SNR $\sim 100$. Image credit: Passegger et al. (2016), Fig.2, reproduced with permission, (c)ESO. 

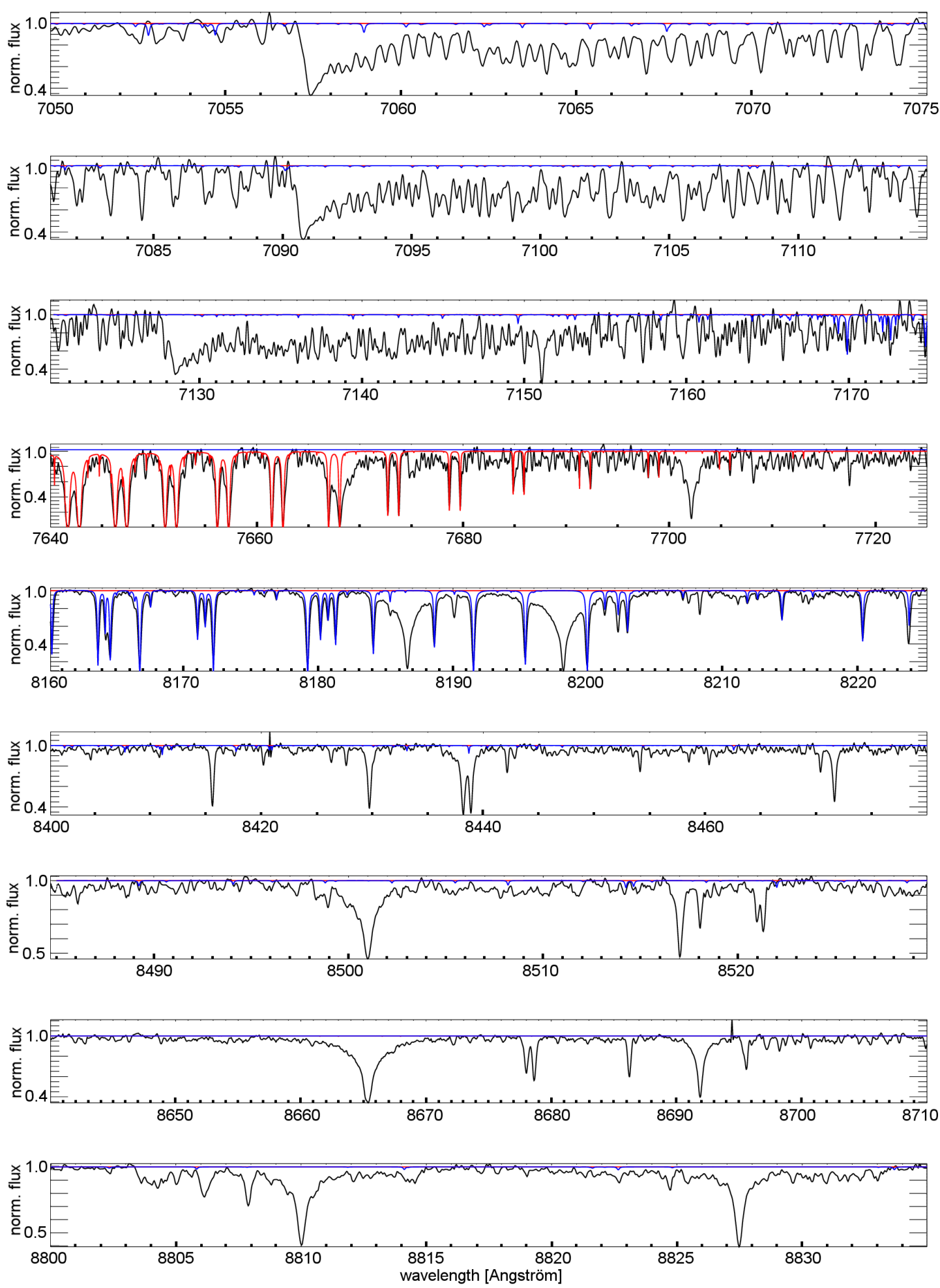

Figure 2.9.: FEROS spectrum of HD 285968 (M2 V, black) together with modelled telluric lines of $\mathrm{O}_{2}$ (red) and $\mathrm{H}_{2} \mathrm{O}$ (blue). Lines of other molecules can be neglected within this wavelength range. 


\subsubsection{The near-infrared regime}

In the framework of this thesis the fiasco-code was used to determine stellar parameters from the near-infrared (NIR) J-, H-, and K-bands for comparison with results obtained from the visual wavelength regime. I used spectra from TripleSpec obtained by RojasAyala et al. (2012) in the NIR, and X-Shooter spectra in the visible and NIR from the ESO Archive for direct comparison of parameters determined from the two wavelength regions. The TripleSpec spectra are corrected for telluric absorption; for the X-Shooter spectra I used the Molecfit-routine described below.

To identify lines sensitive to the stellar parameters $T_{\text {eff }}, \log g$ and $[\mathrm{Fe} / \mathrm{H}]$ in the NIR, the J-, H- and K-bands were plotted together with PHOENIX-ACES models for different parameters. For this I used TripleSpec spectra, since these spectra were already corrected for telluric lines using a telluric standard A-type star. The procedure allowed the study of changes in line shape with changing parameters. The lines found to be suitable for fitting are presented in Table 2.3. During investigation of the K-band, I found that the PHOENIX-ACES models are having problems to reproduce the correct depth of some lines. To illustrate this, Fig. 2.10 shows the spectrum of HIP 12961 with two different models. Note that these models are not fits to the spectrum, but only overplotted for comparison. In the upper panel the model has the same parameters as determined by Rojas-Ayala et al.(2012); however, the observed spectrum has much deeper lines. In the lower panel the model has a metallicity of $[\mathrm{Fe} / \mathrm{H}]=1.0$. Most of the lines are now even too deep, except for the Ca triplet around $2.263 \mu \mathrm{m}$, which is still too shallow. For this reason the triplet was excluded from the fit.

Table 2.3.: Wavelength ranges for fitting parameters in the NIR.

\begin{tabular}{llc}
\hline \hline Band & Line & $\lambda[\mu \mathrm{m}]$ \\
\hline $\mathrm{J}$ & $\mathrm{Fe}$ & 1.17 \\
& & 1.19 \\
& $\mathrm{~K}$ & 1.179 \\
& & 1.242 \\
& & 1.251 \\
& $\mathrm{Si}$ & 1.198 \\
\hline $\mathrm{H}$ & $\mathrm{Al}$ & 1.673 \\
& & 1.676 \\
& $\mathrm{Mg}$ & 1.711 \\
\hline $\mathrm{K}$ & $\mathrm{Si} / \mathrm{Ti}$ & 2.18 \\
& & 2.19 \\
& $\mathrm{Na}$ & 2.205 \\
& $\mathrm{Mg}$ & 2.28 \\
& $\mathrm{CO}$ & $>2.32$ \\
\hline
\end{tabular}



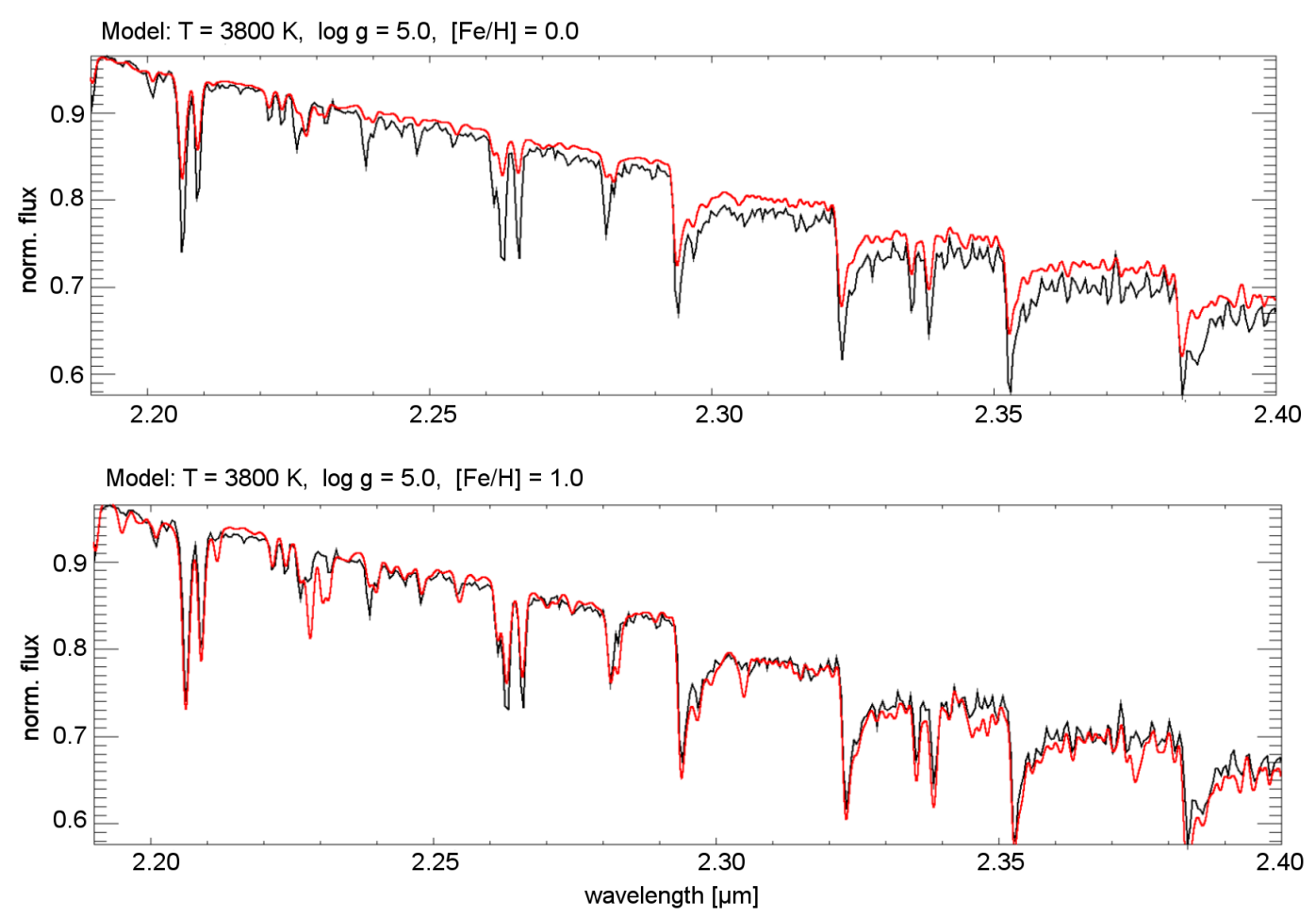

Figure 2.10.: Spectrum of HIP 12961 (black) taken with TripleSpec together with 2 PHOENIX models (red, no fit). Upper panel: Model parameters consistent with parameters found by Rojas-Ayala et al. (2012). Lower panel: Same temperature and $\log g$ as upper model, $[\mathrm{Fe} / \mathrm{H}]=1.0$

\subsubsection{Telluric correction}

The NIR regime is highly affected by molecular lines from Earth's atmosphere, so-called telluric lines. Between regions of high telluric contamination, less contaminated "windows" appear, which are identified as "bands", e.g. J-, H-, and K-bands (1.0-1.4 $\mu \mathrm{m}$, $1.5-1.8 \mu \mathrm{m}$ and $2.0-2.5 \mu \mathrm{m}$, respectively). Nevertheless, the bands themselves are still influenced by a vast amount of telluric lines, which makes telluric corrections essential. There are multiple ways of correcting a spectrum for telluric lines. A widely used method is to observe a telluric standard star together with the target star. This telluric standard is preferably observed shortly before or after the target star and angularly close on sky in order to minimise temporal and spatial variations in the atmosphere. Usually these stars are of early spectral types because they show only a few stellar features and therefore telluric lines are easily recognisable. This method was chosen to correct spectra taken with TripleSpec by observing a telluric standard A0 V star, which is mainly featureless in the NIR except for some hydrogen absorption lines. The hydrogen lines were removed by using a high-resolution model of Vega, leaving only telluric absorption in the spectrum. The observed spectrum of the target star was then divided by the telluric 
spectrum.

Since the method of observing telluric standards sometimes involves difficulties (e.g. investment of more telescope time, lack of suitable stars close to the target, fast change of atmospheric conditions or insufficient signal-to-noise ratio of the telluric spectrum), synthetic transmission models have been created to fit telluric features by using radiative transfer codes for atmospheric research. Using these codes Smette et al. (2015) and Kausch et al. (2015) presented the ESO Molecfit-routine. Molecfit works with state-ofthe-art radiative transfer models to calculate the atmospheric absorption for the time and location of observation. Therefore it uses atmospheric data, consisting of a reference atmospheric profile, modelled 3D profiles obtained by the Global Data Assimilation System (GDAS) and on-site measurements from the Astronomical Site Monitor (ASM). These data, merged into an atmospheric profile together with a molecular line database, are handed to the radiative transfer code LNFL/LBLRTM (Clough et al., 2005) to calculate radiance and transmission spectra. Involving instrumental properties and line spread functions, a Levenberg-Marquardt algorithm adjusts the atmospheric spectrum for every molecule inside the specified fitting regions and gives the observed spectrum corrected for the best-fit atmospheric model. I held on to the fitting regions described in Kausch et al. (2015) for correcting X-Shooter spectra and added some more to especially clean the J-band. Table 2.4 summarises all wavelength ranges used for fitting. Fig. 2.11 shows the observed and telluric corrected spectrum of Gl 393.

Table 2.4.: Wavelength ranges (air) used for fitting the X-Shooter NIR spectra

\begin{tabular}{lll}
\hline \hline No. & $\lambda_{\min }[\mu \mathrm{m}]$ & $\lambda_{\max }[\mu \mathrm{m}]$ \\
\hline 1 & 1.100 & 1.130 \\
2 & 1.147 & 1.155 \\
3 & 1.260 & 1.267 \\
4 & 1.300 & 1.310 \\
5 & 1.320 & 1.330 \\
6 & 1.470 & 1.485 \\
7 & 1.800 & 1.810 \\
8 & 2.060 & 2.070 \\
9 & 2.350 & 2.360 \\
\hline
\end{tabular}




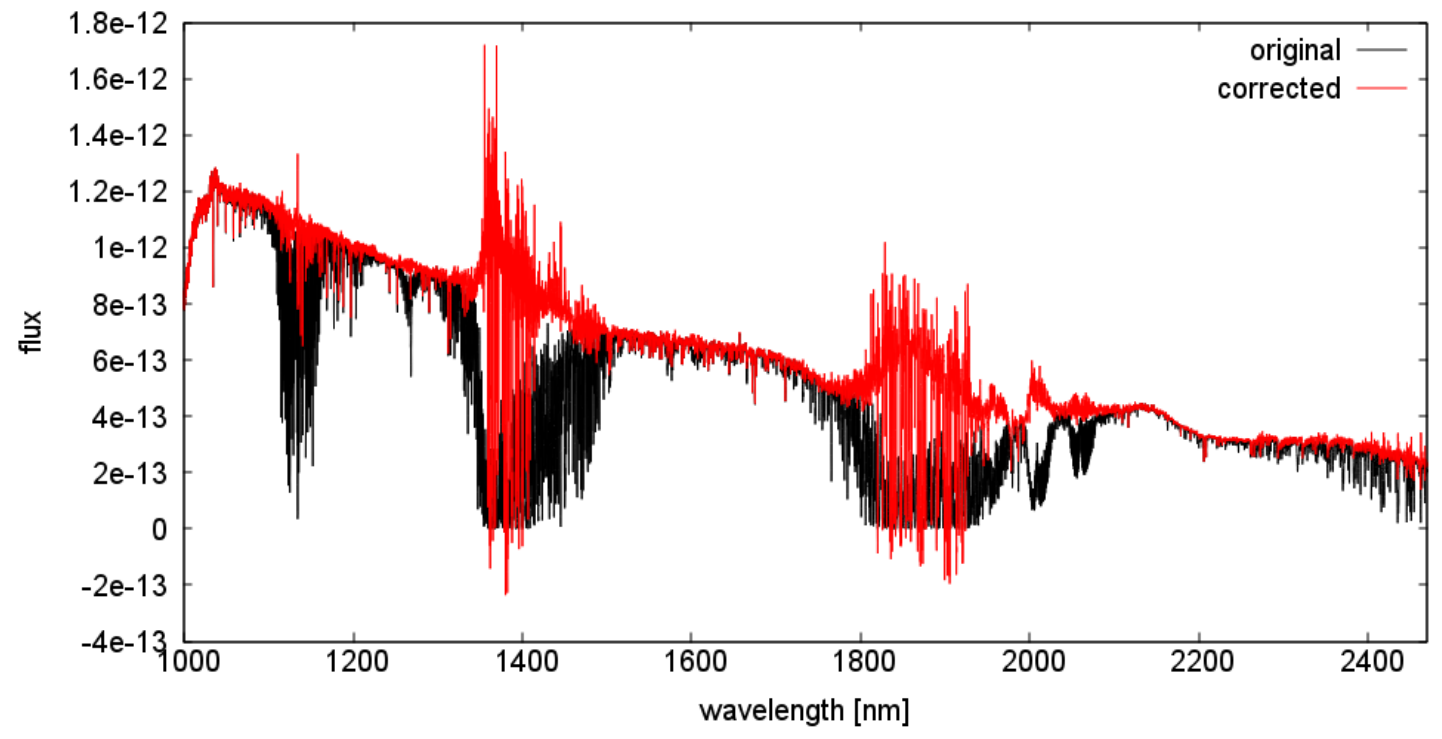

Figure 2.11.: Spectrum of G1 393 observed with X-Shooter (black), the spectrum after telluric correction with Molecfit is plotted in red. 


\section{Data}

In order to characterise the CARMENES target sample before the start of the survey, all target stars were observed by the CARMENES team with other high-resolution spectrographs, namely CAFE, FEROS and HRS. In total 3499 spectra of 593 stars were taken. Table 3.1 summarises these observations. The first six months of data taken with CARMENES are also included. Additionally, I inspected NIR spectra taken with TripleSpec and X-Shooter. Fig. 3.1 shows the signal-to-noise distribution of the spectra from CAFE, FEROS, HRS and CARMENES with measured $v \sin i$.

Table 3.1.: Summary of observations.

\begin{tabular}{|c|c|c|c|c|c|c|}
\hline Instrument & $\begin{array}{l}\text { \# Spectra } \\
\text { observed }\end{array}$ & $\begin{array}{c}\text { \# Stars } \\
\text { observed }\end{array}$ & $\begin{array}{l}\text { \# Spectra } \\
\text { analysed }\end{array}$ & $\begin{array}{c}\text { \# Stars } \\
\text { analysed }\end{array}$ & $\begin{array}{l}\text { \# Stars } \\
\text { good fit }\end{array}$ & $\begin{array}{c}\text { Observing } \\
\text { period }\end{array}$ \\
\hline CAFE & 927 & 297 & 681 & 267 & 153 & $\begin{array}{c}01 / 2013 \text { to } \\
09 / 2014\end{array}$ \\
\hline FEROS & 651 & 265 & 562 & 248 & 182 & $\begin{array}{c}12 / 2012 \text { to } \\
07 / 2014\end{array}$ \\
\hline HRS & 160 & 46 & 147 & 41 & 15 & $\begin{array}{c}09 / 2011 \text { to } \\
06 / 2013\end{array}$ \\
\hline CARMENES & 1761 & 251 & 1738 & 245 & 179 & $\begin{array}{c}01 / 2016 \text { to } \\
06 / 2016\end{array}$ \\
\hline Total & 3499 & 593 & 3128 & 543 & 356 & $\ldots$ \\
\hline TripleSpec & 19 & 19 & 19 & 19 & 19 & 2007 to 2010 \\
\hline X-Shooter & 13 & 13 & 13 & 13 & 13 & $\begin{array}{c}02 / 2010 \text { to } \\
03 / 2012\end{array}$ \\
\hline
\end{tabular}

All observed stars were processed by the first part of the fiasco-code described in Section 2. After that the resulting plots were inspected manually. Bad spectra, resulting from very low SNR or errors during the observation, were discarded. The remaining number of spectra and stars is shown in Table 3.1 with the keyword "analysed". The total sum of both columns "\# Stars" is not equal to the actual sum of the numbers because several stars were observed with two or more spectrographs. This is presented in Table 3.2 . 
Table 3.2.: Sample overlap

\begin{tabular}{lcccc}
\hline \hline & CAFE & FEROS & HRS & CARMENES \\
CAFE & - & 32 & 1 & 83 \\
FEROS & - & - & 2 & 44 \\
HRS & - & - & - & 4 \\
\hline
\end{tabular}

Additionally, 2 stars were observed with CAFE, HRS and CARMENES and 41 stars were observed with CAFE, FEROS and CARMENES. The results of the overlapping sample between CAFE, FEROS and CARMENES are analysed in more detail in Section 5.1. In the following I will shortly describe the spectrographs used for observations and give some more details about the spectra taken.

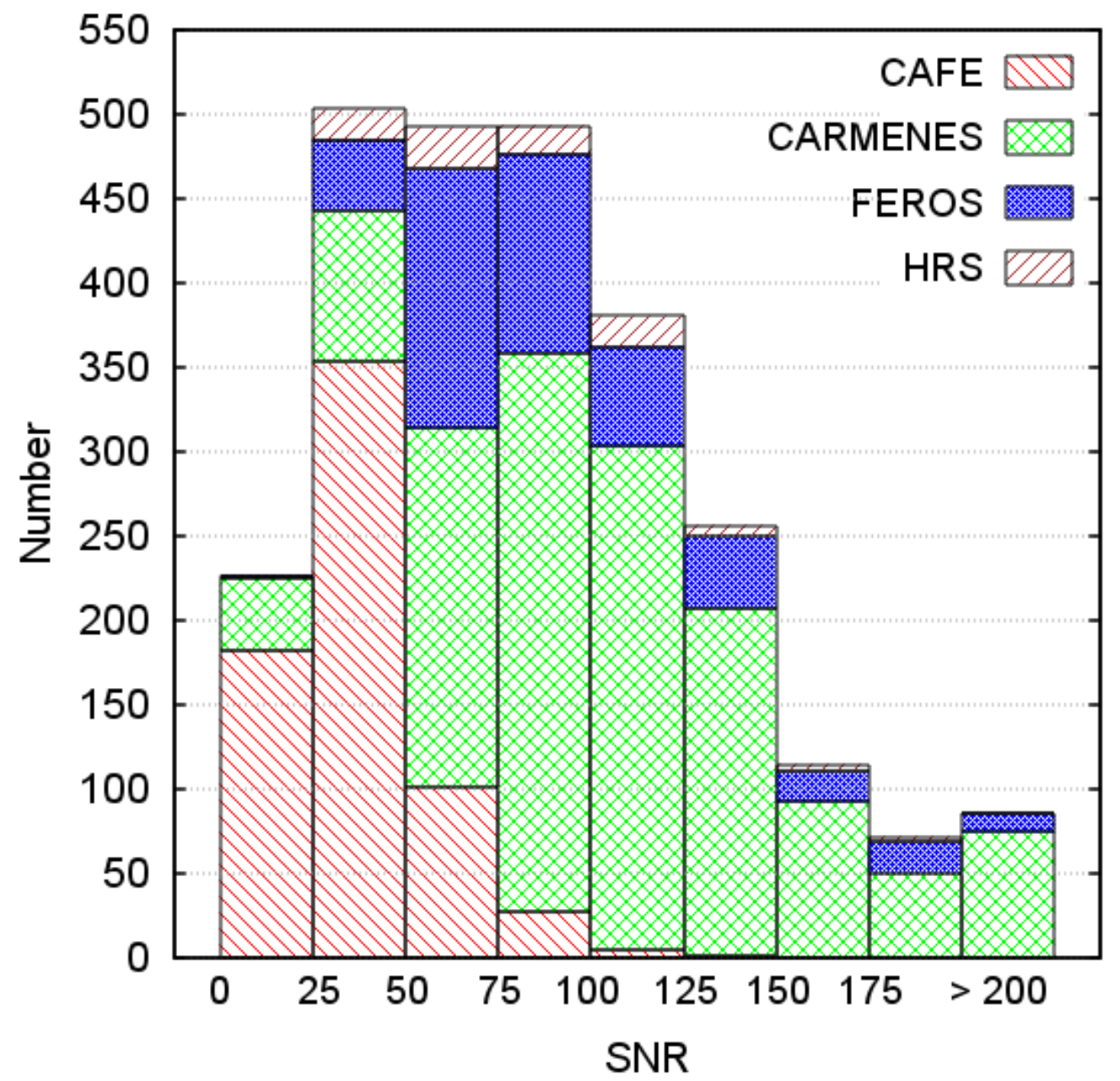

Figure 3.1.: Signal-to-Noise distribution for all spectra with $v \sin i$ measurements from CAFE, FEROS, HRS and CARMENES. 
CAFE The Calar Alto Fiber-fed Échelle spectrograph (CAFE) is mounted at the $2.2 \mathrm{~m}$ telescope at Calar Alto Observatory in Spain. It covers a wavelength range between 3960-9500 $\AA$ with a resolution of $\mathrm{R} \approx 62000$ (Aceituno et al., 2013). Due to gaps between the orders some single lines cannot be observed, i.e. the second $\mathrm{Na} \lambda 8197$ $\AA$ line and the $\epsilon$ - TiO bandhead at $\lambda 8437 \AA$. The spectra were reduced using the IDL package REDUCE (Piskunov \& Valenti, 2002) in combination with the flat-relative optimal extraction (FOX) by Zechmeister et al. (2014). Spectra observed until May 2014 have been reduced by Lamert (2014), spectra observed after that date were reduced by Schöfer (2015).

FEROS The Fiber-fed Extended Range Optical Spectrograph (FEROS) is located at the $2.2 \mathrm{~m}$ telescope at the European Southern Observatory in La Silla, Chile. It has a wavelength coverage from $3600 \AA$ to $9200 \AA$ with a resolution of $\mathrm{R} \approx 48000$ (Kaufer et al., 1997). Data reduction was done automatically by the FEROS Data Reduction System. The spectra are available in the ESO Public Archive.

HRS The High-Resolution Spectrograph (HRS) is located at the $9.2 \mathrm{~m}$ Hobby-Eberly telescope at McDonald Observatory in Texas, United States. Using two CCD detectors it covers a wavelength range between $4200-11000 \AA$; the range from 6900 to $7065 \AA$ falls in the gap between the two CCDs and is therefore not covered (Tull et al., 1998). An adjustable slit allows for three different resolutions; our observations were done with $\mathrm{R} \approx 60000$. Data reduction was the same as for CAFE done by Lamert (2014).

CARMENES In addition, I analysed 1738 visual spectra taken from the first six months of the survey from January 1st 2016 to June 30th 2016 for comparison of parameters. The spectra were automatically reduced by the CARMENES pipeline CARACAL (Zechmeister et al., in prep.).

TripleSpec I analysed 19 spectra taken by Rojas-Ayala et al. (2012) with TripleSpec, located at the 200 inch Hale telescope on Palomar (Herter et al., 2008). The spectra were observed between 2007 and 2010 with a resolution of 2700 . The spectral coverage of the spectrograph ranges from 1.0 to $2.4 \mu \mathrm{m}$. For telluric correction an A-star was used; the cleaned spectra are of good quality and almost free of telluric remnants. K-band spectra are online available on VizieR (Ochsenbein et al., 2000). I obtained spectra of the Jand H-bands from private communication with Barbara Rojas-Ayala. Since the spectra were flux-calibrated, the overall shape of the energy distribution is recovered and can be used to constrain temperature, which is not possible for the CAFE, FEROS, HRS and CARMENES spectra.

X-Shooter The X-Shooter spectrograph is mounted at the Unit Telescope 2 at the Very Large Telescope on Paranal in Chile, providing spectra in the UBV, VIS and NIR wavelength ranges. I made use of 13 ESO public archive spectra observed with XShooter in the VIS and NIR during observing runs 084.D-0795, 086.D-0248, 088.D0096, 088.D-0556 and 385.D-0200, between 02/2010 and 03/2012. The exposure times 
range from $1 \mathrm{sec}$ to $184 \mathrm{sec}$, the spectral resolution lies between 5400 and 18000 . The NIR spectra cover a wavelength range from 994 to $2479 \mathrm{~nm}$. I used the reduced data coming from the ESO PHASE3 data reduction pipeline.

The Molecfit-routine was used to correct the J-, H- and K-bands for telluric absorption. A comparison with uncleaned spectra shows that Molecfit weakens the line depths of some lines in the J- and H-bands. Unfortunately, I found that for X-Shooter, the Kband spectra show an unusual "bump" in flux between 2.1 and $2.2 \mu \mathrm{m}$, which can be seen Fig. 3.2. It results either from saturation or an incorrect account of the instrument response function, since calibration spectra are not taken every night. This leaves the Jand $\mathrm{H}$-bands for analysis.

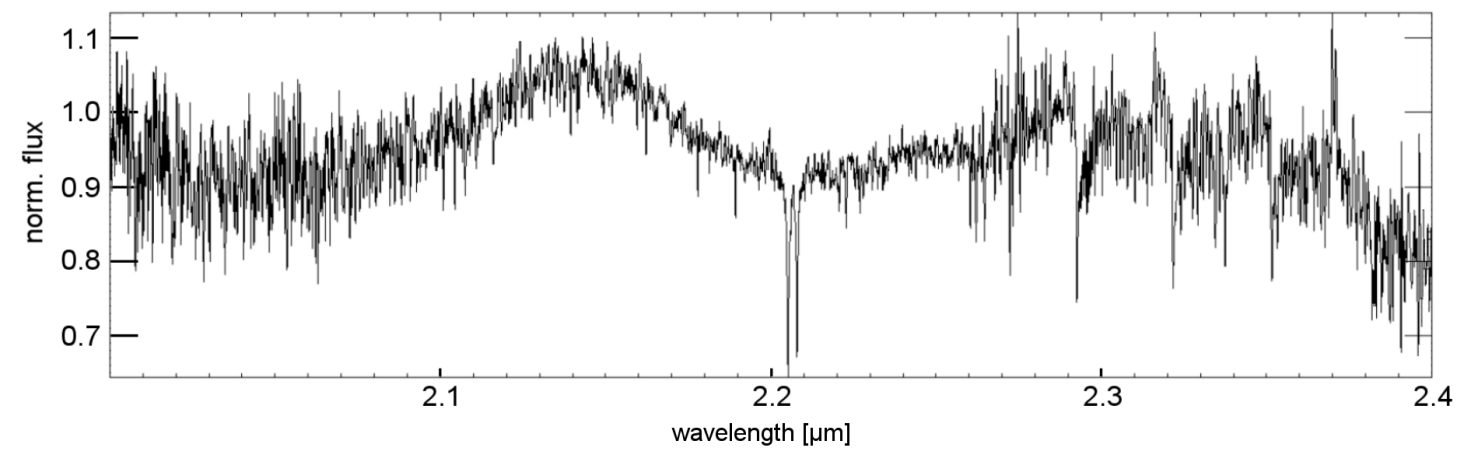

Figure 3.2.: Spectrum of G1 447 observed with X-Shooter showing the "bump" between 2.1 and $2.2 \mu \mathrm{m}$ in the K-band. 


\section{Results}

In this chapter I will present results from CAFE, FEROS and HRS for the science preparation of CARMENES and results from the first six months of CARMENES data. A full list of the analysed stars with determined parameters is given in the Appendix in Table A.1. I will also provide results from TripleSpec spectra in the NIR and from X-Shooter spectra in the NIR and VIS.

\subsection{Results from CAFE, FEROS and HRS}

For the CARMENES science preparation I analysed 1390 spectra (484 stars) taken with CAFE, FEROS and HRS. I obtained good results for 310 stars. To identify a good fit, I inspected all fits and put a $\chi^{2}$ limit based on the overall quality of the spectra from each spectrograph. The quality is mainly constrained by the SNR (shown in Fig. 3.1), but is also influenced by rotational velocities. The $\chi^{2}$ limits are 3 for FEROS, 4 for HRS, and 5 for CAFE. Only results below these limits are listed in the Appendix in Table A.1. Jeffers et al. (submitted) determined $v \sin i$ from each spectrum of the stars. For the 72 stars that they do not provide $v \sin i$ measurements for, I assigned a value of $3 \mathrm{kms}^{-1}$, since this is the minimum $v \sin i$ detectable with a spectral resolution of 82000 (i.e. CARMENES). These stars are included in the Appendix in Table A.2, but not in the plots of Figs. 4.1 and 4.5. The histogram distribution for the parameters is presented in Fig. 4.1. For the majority of the sample $T_{\text {eff }}$ lies between 3200 and $3800 \mathrm{~K}$, as expected for spectral type M 0.0 to M 5.0. There is a higher temperature tail, which might result from misidentified late $\mathrm{K}$ stars left in the sample. Values for the surface gravity $\log g$ are between 4.5 and 5.5 dex, with the peak of the distribution around 5.0 dex. Since I derive $\log g$ from evolutionary models for a given temperature and metallicity, the results are not expected to spread outside of these values. The metallicity distribution shows mainly sub-solar values with a small tail towards super-solar metallicities. Figs. 4.2,4.4 show final plots of typical spectra for each spectrograph. 

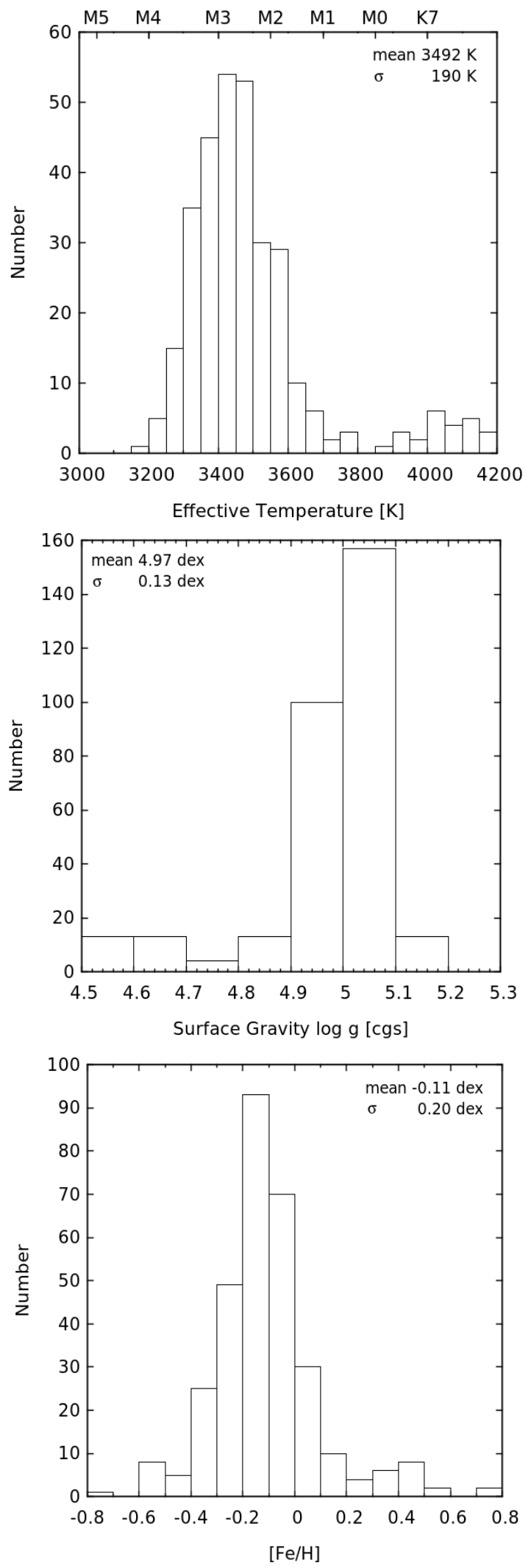

Figure 4.1.: Histogram distributions for the determined parameters from the CAFE, FEROS and HRS stars. Top panel: effective temperature; Middle panel: surface gravity; Lower Panel: metallicity. 

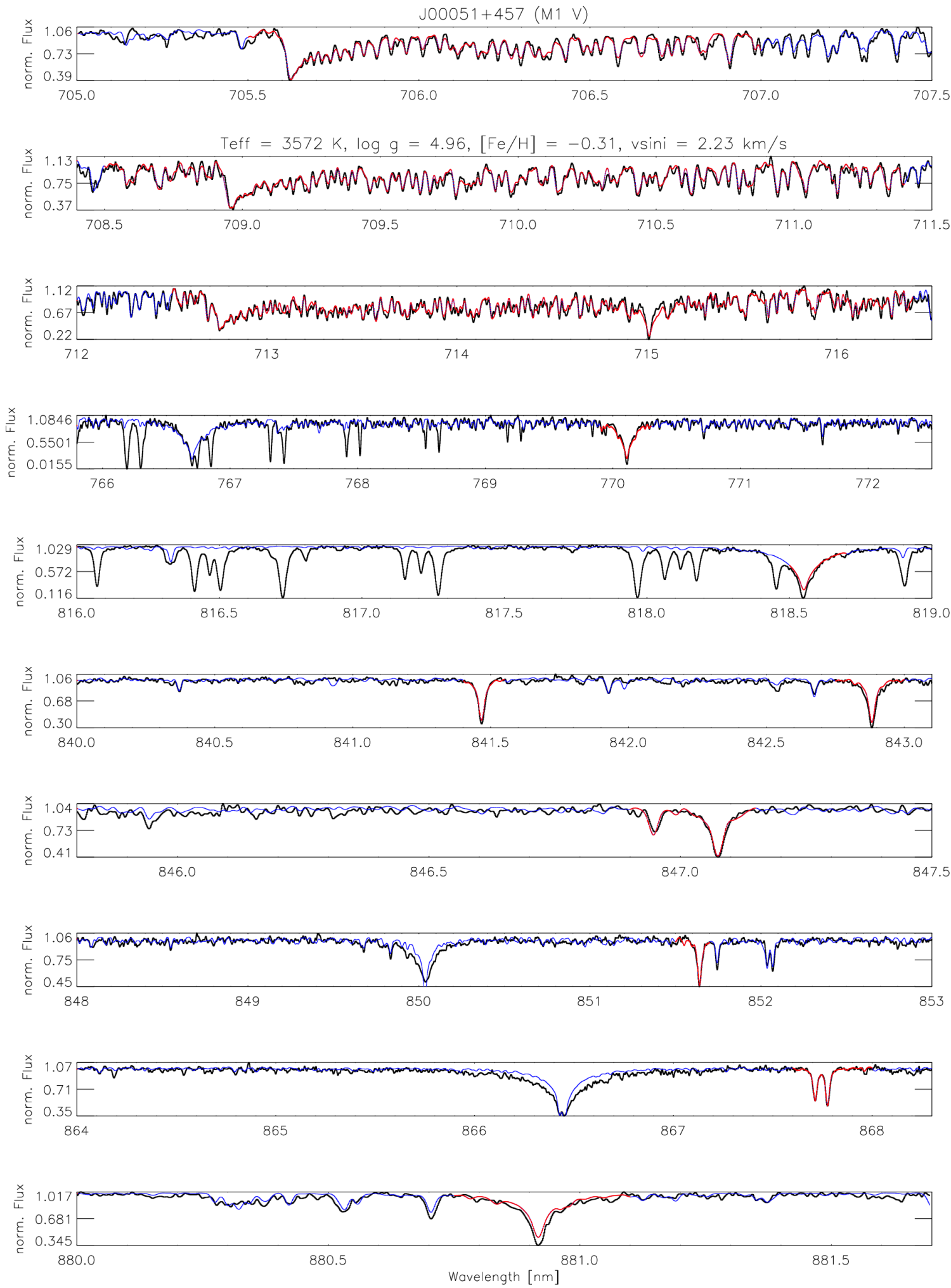

Figure 4.2.: Typical spectrum of an M1 V star observed with CAFE (black). The best fit model is plotted in blue, with the fitting regions marked in red. The best fit parameters can be found on top of the second panel. 

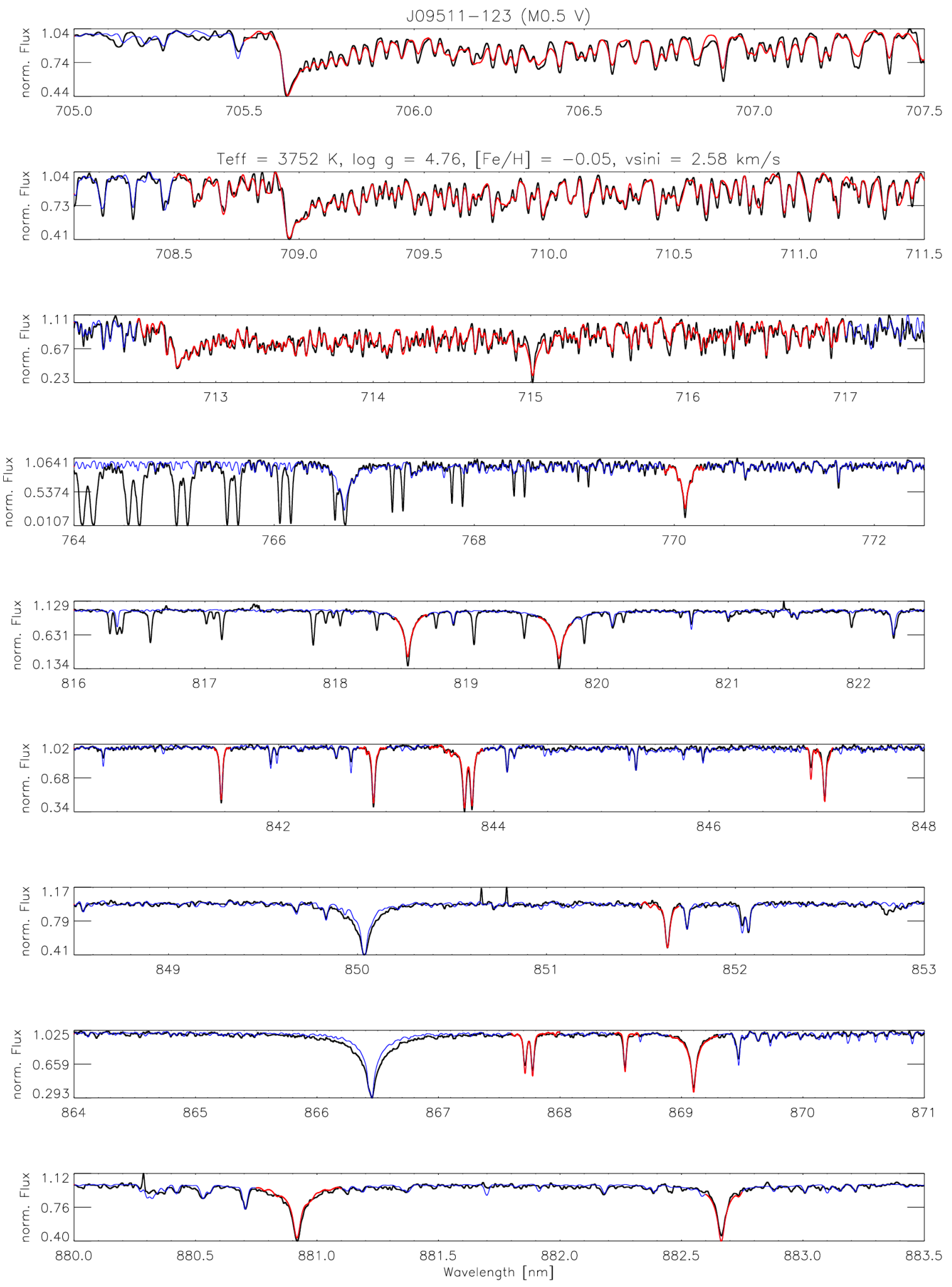

Figure 4.3.: Typical spectrum of an M0.5 V star observed with FEROS (black). The best fit model is plotted in blue, with the fitting regions marked in red. The best fit parameters can be found on top of the second panel. 
$\mathrm{J} 21057+502(\mathrm{M} 3.5 \mathrm{~V})$
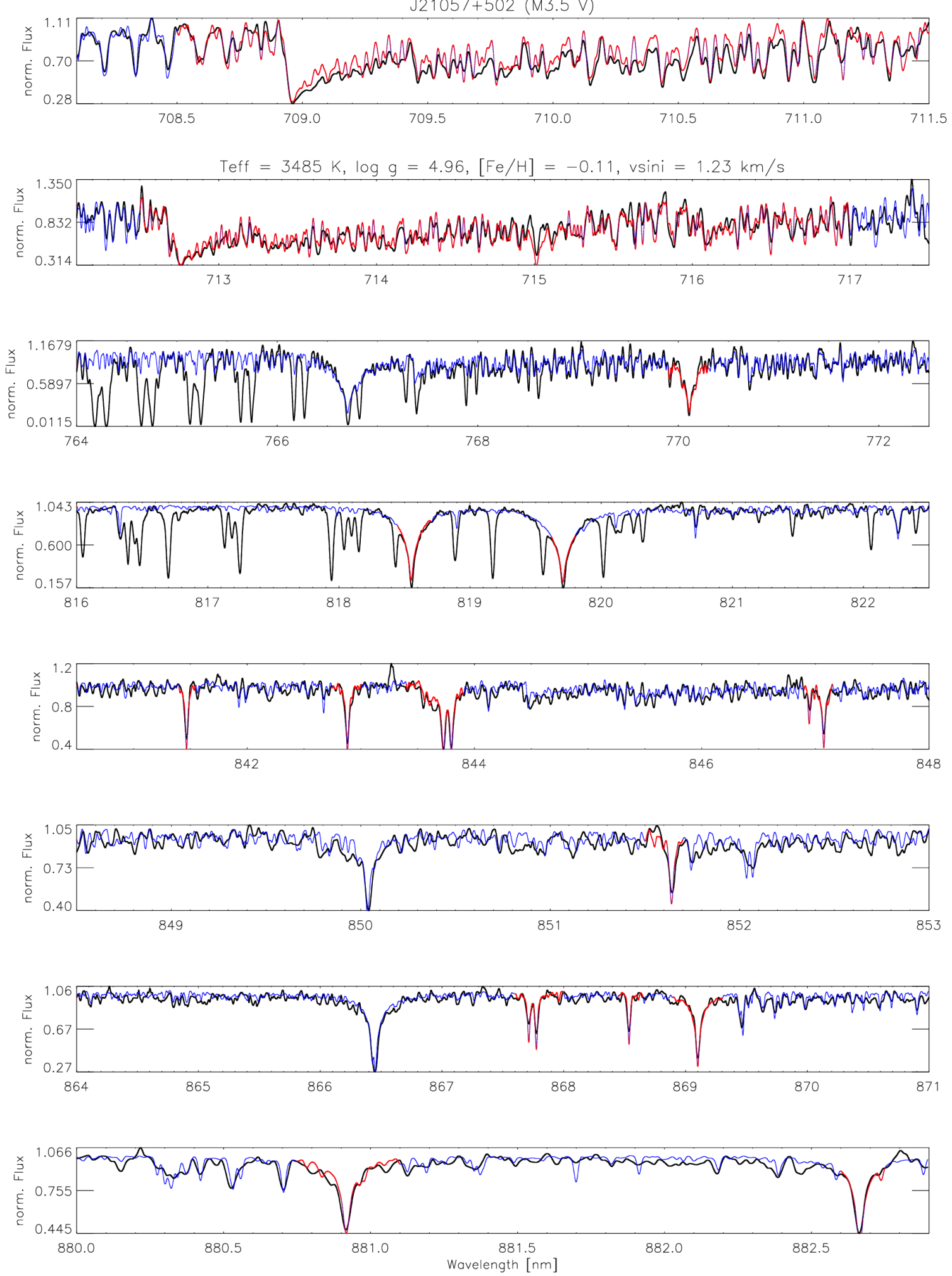

Figure 4.4.: Typical spectrum of an M3.5 V star observed with HRS (black). The best fit model is plotted in blue, with the fitting regions marked in red. The best fit parameters can be found on top of the second panel. 


\subsection{Results from CARMENES}

I inspected the first six months of data taken with CARMENES. From 1761 observed spectra I analysed 1738 spectra and got good results for 144 stars. As for the CAFE, FEROS and HRS sample I accounted for the $v \sin i$ measured by Jeffers et al. (submitted) for each star. 70 stars do not have a $v \sin i$ value; for those I again assumed a default value of $3 \mathrm{kms}^{-1}$. As for the CAFE, FEROS and HRS spectra, I set a $\chi^{2}$ limit based on the quality of the fit. For the CARMENES results this limit is 3.5. I also analysed template spectra for 44 stars. These templates are products of the SERVAL pipeline to measure RV shifts (Zechmeister et al., in prep.). For stars with at least 5 observations all single spectra are co-added to increase SNR. Fig. 4.5 presents histogram distributions for the stellar parameters. The distributions are very similar to the ones from CAFE, FEROS and HRS, which can be also seen from the mean and $\sigma$ values shown in each panel. An example spectrum of a typical star observed with CARMENES together with the best fit model is plotted in Fig. 4.6. 

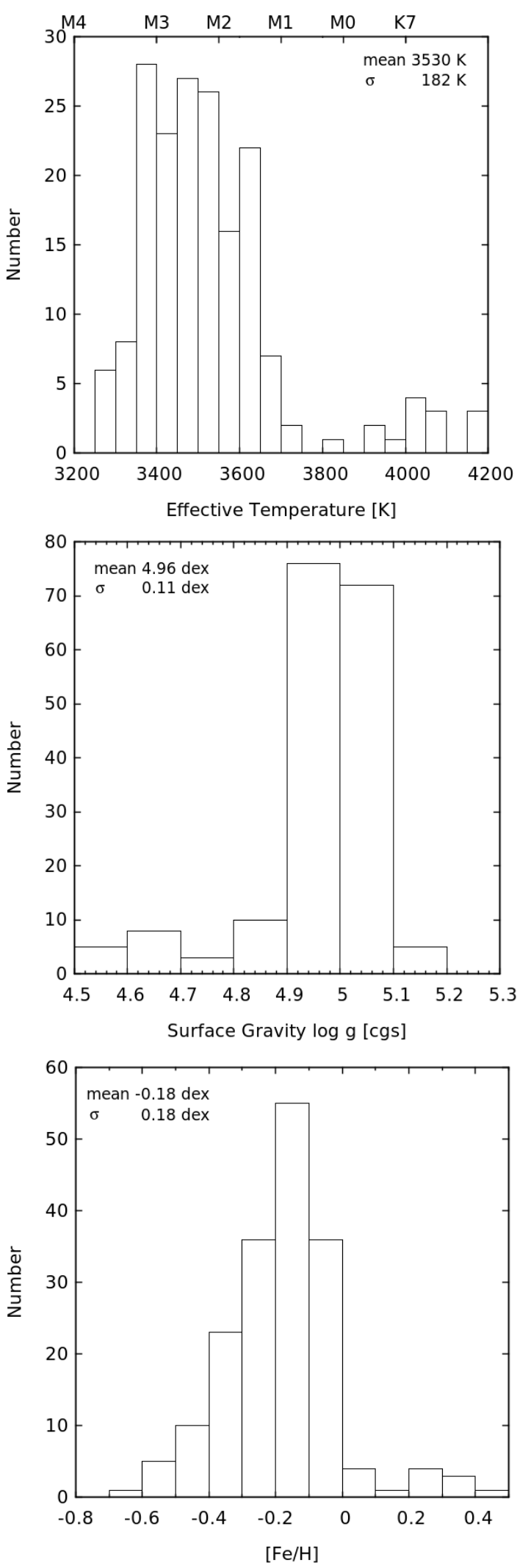

Figure 4.5.: Histogram distributions for the determined parameters from CARMENES stars. Top panel: effective temperature; Middle panel: surface gravity; Lower Panel: metallicity. 

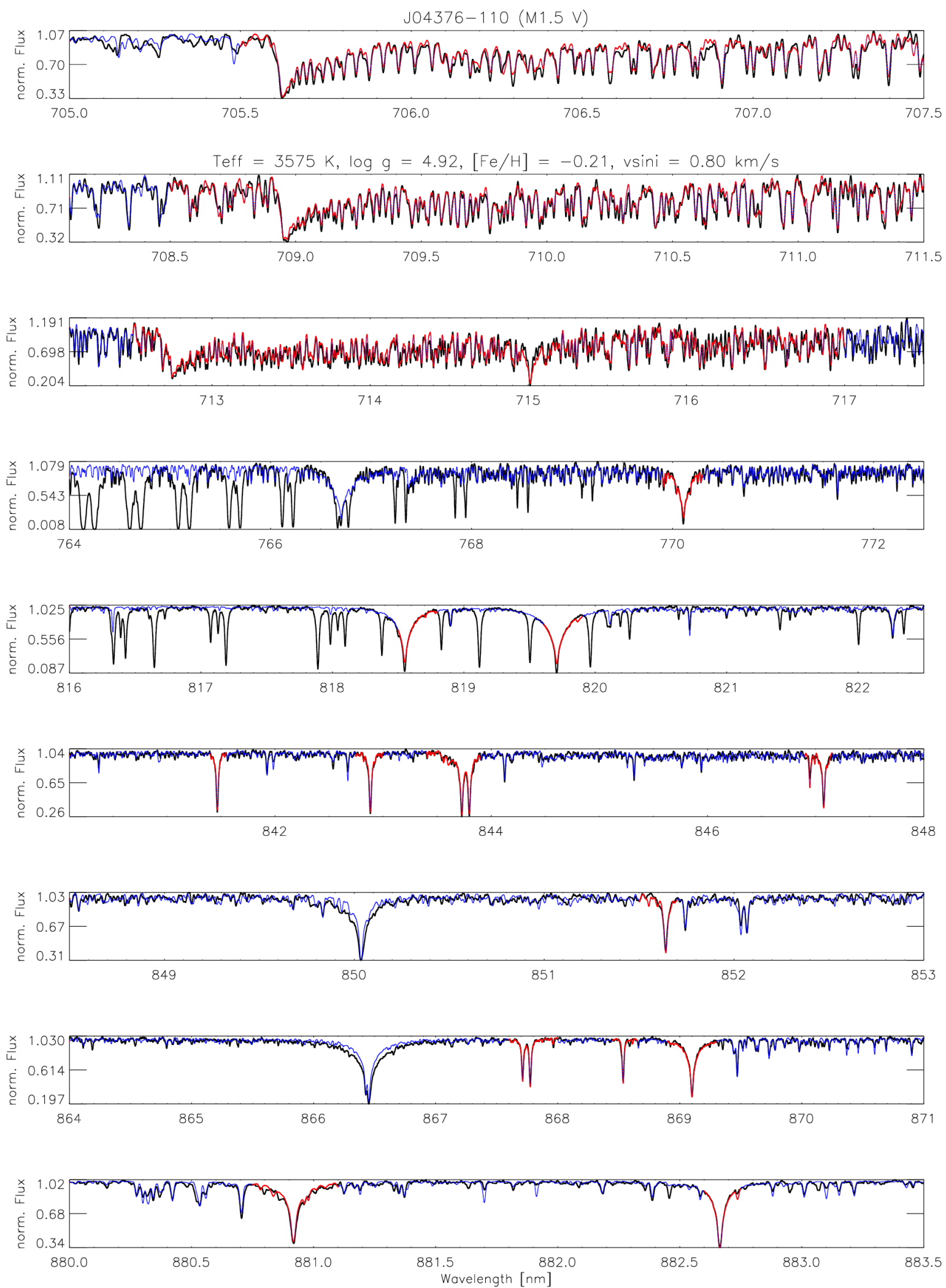

Figure 4.6.: Typical spectrum of an M1.5 V star observed with CARMENES (black). The best fit model is plotted in blue, with the fitting regions marked in red. The best fit parameters can be found on top of the second panel. 


\subsection{Results from TripleSpec}

The main purpose of investigating TripleSpec spectra is to compare accurate results from Rojas-Ayala et al. (2012) with results from my method in the NIR and find possible inconsistencies. I investigated spectra of $19 \mathrm{M}$ dwarfs taken with TripleSpec. The spectra have been used by Rojas-Ayala et al. (2012) to derive effective temperatures and metallicities from the $\mathrm{H}_{2} \mathrm{O}-\mathrm{K} 2$ index and equivalent widths of $\mathrm{NaI}$ and $\mathrm{CaI}$ in the NIR K-band. Fig. 4.7 shows an example spectrum with the best fit model for each wavelength band. The lines and regions used for fitting are marked in red and the lines are identified. Table 4.1 presents my results from the J-, H-, and K-band, as well as results from RojasAyala et al. (2012). In cases where the M-dwarf has an FGK binary companion, the metallicities of the primary are given from literature. A comparison of the results for each star is plotted in Fig. 4.8. Since the temperature is constrained by the overall energy distribution of the spectrum, the values mostly agree with Rojas-Ayala et al. (2012), except for some rare outliers such as G1 908. Also, $\log g$ derived from the different bands is mostly consistent within the expected range of 4.5-5.5 dex.
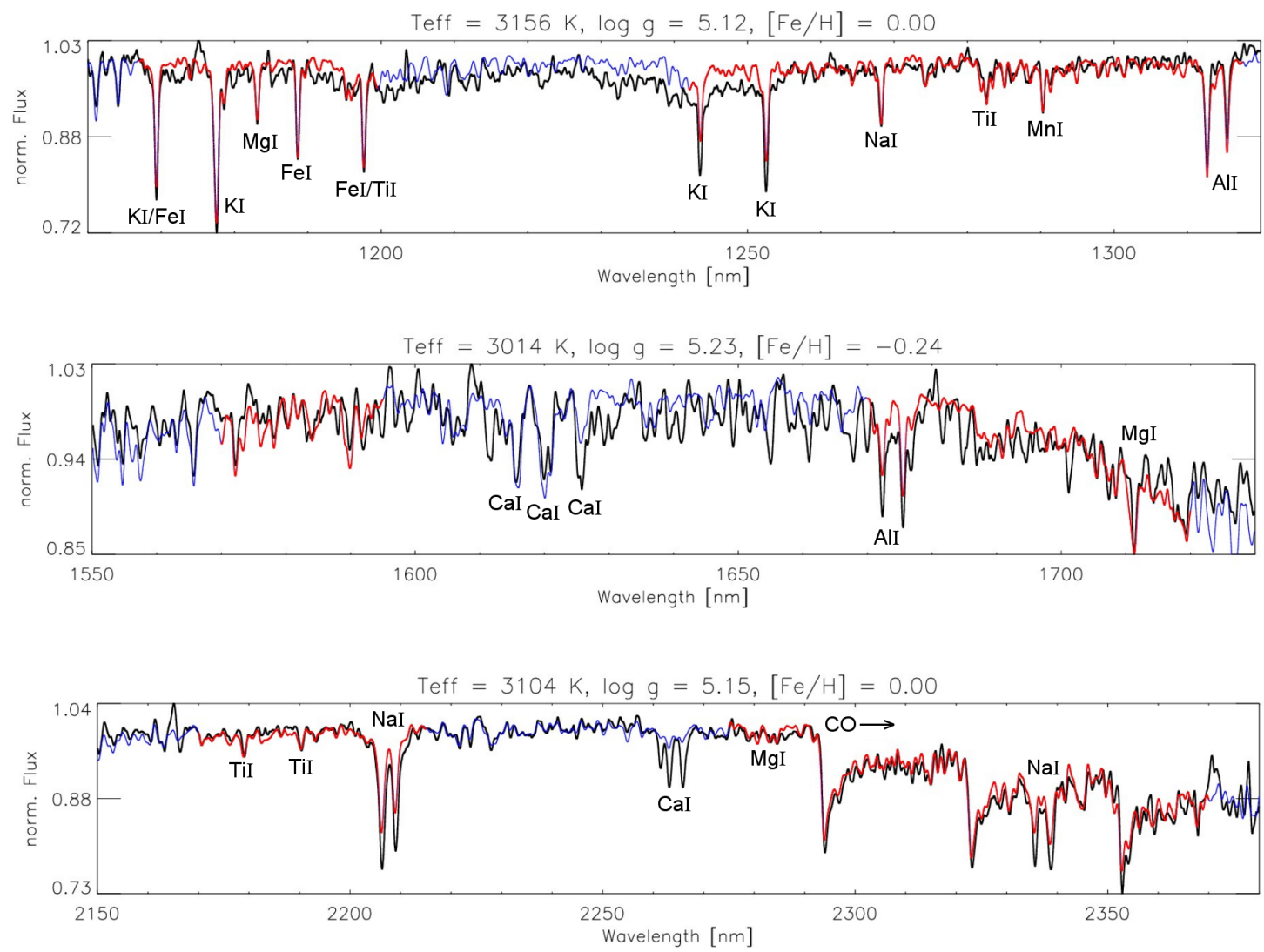

Figure 4.7.: Spectrum of G1 1214 observed with TripleSpec (black) and the best fit model in blue. Regions used for $\chi^{2}$-minimisation are marked in red. Top panel: J-band; Middle panel: H-band; Bottom panel: K-band. 
Table 4.1.: Results from TripleSpec spectra in the J-, H- and K-bands, together with values from Rojas-Ayala et al. (2012) (RA12). The last column provides metallicities measured for the primary companion (FGK star, if present) from literature.

\begin{tabular}{|c|c|c|c|c|c|c|c|c|c|c|c|c|}
\hline & \multicolumn{3}{|c|}{$\overline{\mathrm{J}}$} & \multicolumn{3}{|c|}{$\overline{\mathrm{H}}$} & \multicolumn{3}{|c|}{$\overline{\mathrm{K}}$} & \multicolumn{2}{|c|}{ RA12 } & \multirow{2}{*}{$\begin{array}{c}\text { Primary } \\
{[\mathrm{Fe} / \mathrm{H}]}\end{array}$} \\
\hline Name & $\begin{array}{c}T_{\text {eff }} \\
\pm 94\end{array}$ & $\begin{array}{c}\log g \\
\pm 0.33\end{array}$ & $\begin{array}{c}{[\mathrm{Fe} / \mathrm{H}]} \\
\pm 0.34\end{array}$ & $\begin{array}{c}T_{\text {eff }} \\
\pm 18\end{array}$ & $\begin{array}{c}\log g \\
\pm 0.33\end{array}$ & $\begin{array}{c}{[\mathrm{Fe} / \mathrm{H}]} \\
\pm 0.19\end{array}$ & $\begin{array}{c}T_{\text {eff }} \\
\pm 179\end{array}$ & $\begin{array}{c}\log g \\
\pm 0.32\end{array}$ & $\begin{array}{c}{[\mathrm{Fe} / \mathrm{H}]} \\
\pm 0.38\end{array}$ & $T_{\text {eff }}$ & $\begin{array}{l}{[\mathrm{Fe} / \mathrm{H}]} \\
\pm 0.17\end{array}$ & \\
\hline Gl 273 & 3465 & 4.85 & 0.32 & 3293 & 5.20 & -0.65 & 3236 & 5.08 & 0.07 & $3239 \pm 26$ & -0.17 & - \\
\hline Gl 285 & 3415 & 4.74 & 0.99 & 3207 & 5.18 & -0.39 & 3002 & 5.18 & 0.03 & $3045 \pm 59$ & 0.40 & - \\
\hline Gl 297.2B & 3544 & 5.21 & -1.00 & 3935 & 4.88 & -0.89 & 3525 & 4.84 & 0.14 & $3585 \pm 20$ & -0.04 & $-0.09 \pm 0.09^{1}$ \\
\hline Gl 402 & 3299 & 5.17 & -0.50 & 3352 & 5.18 & -0.68 & 3095 & 5.25 & -0.50 & $3334 \pm 23$ & 0.20 & - \\
\hline Gl 406 & 2711 & 4.67 & -0.33 & 2995 & 5.27 & -0.47 & 2895 & 5.25 & -0.13 & $2887 \pm 20$ & 0.18 & - \\
\hline Gl 447 & 3135 & 5.11 & 0.12 & 3100 & 5.26 & -0.56 & 3030 & 5.15 & 0.13 & $3065 \pm 69$ & -0.01 & - \\
\hline Gl 876 & 3465 & 4.84 & 0.36 & 3325 & 5.16 & -0.51 & 3195 & 5.21 & -0.48 & $3473 \pm 17$ & 0.19 & - \\
\hline Gl 53.1B & 3286 & 5.16 & -0.46 & 3201 & 5.23 & -0.59 & 3425 & 4.82 & 0.63 & $3282 \pm 27$ & 0.21 & $+0.07 \pm 0.12^{1}$ \\
\hline Gl 166C & 3125 & 5.16 & -0.10 & 3001 & 5.20 & -0.08 & 3075 & 5.06 & 0.53 & $2979 \pm 32$ & -0.15 & $-0.28 \pm 0.02^{2}$ \\
\hline Gl 250B & 3175 & 5.12 & 0.00 & 3335 & 5.08 & -0.17 & 3545 & 4.91 & -0.11 & $3569 \pm 20$ & 0.01 & $-0.15 \pm 0.09^{1}$ \\
\hline Gl 324B & 3325 & 5.13 & -0.39 & 3325 & 5.20 & -0.69 & 3085 & 5.25 & -0.50 & $3256 \pm 29$ & 0.31 & $-0.32 \pm 0.07^{1}$ \\
\hline Gl 544B & 3455 & 4.84 & 0.40 & 3111 & 5.24 & -0.50 & 3125 & 5.05 & 0.50 & $3058 \pm 65$ & -0.09 & $-0.20 \pm 0.19^{1}$ \\
\hline GJ 1214 & 3165 & 5.13 & 0.00 & 3014 & 5.23 & -0.24 & 3104 & 5.15 & 0.00 & $3245 \pm 31$ & 0.20 & - \\
\hline GJ 3348B & 3258 & 5.18 & -0.50 & 3213 & 5.23 & -0.65 & 3175 & 5.22 & -0.48 & $3189 \pm 39$ & -0.02 & $-0.22 \pm 0.03^{2}$ \\
\hline Gl 611B & 3216 & 5.12 & -0.09 & 3016 & 5.24 & -0.31 & 3155 & 5.04 & 0.47 & $3051 \pm 61$ & -0.45 & $-0.69 \pm 0.03^{2}$ \\
\hline Gl 908 & 3465 & 4.84 & 0.36 & 3325 & 5.16 & -0.51 & 3195 & 5.21 & -0.49 & $3995 \pm 47$ & -0.59 & - \\
\hline HD46375B & 3765 & 4.57 & 0.55 & 3768 & 4.67 & 0.21 & 3695 & 4.56 & 0.61 & $3663 \pm 15$ & 0.29 & $+0.24 \pm 0.03^{2}$ \\
\hline HIP12961 & 4358 & 4.58 & 0.13 & 4167 & 4.59 & 0.27 & 3844 & 4.59 & 0.47 & $3838 \pm 19$ & 0.01 & - \\
\hline LHS2090 & 2835 & 5.24 & 0.01 & 2945 & 5.27 & -0.38 & 2865 & 5.24 & 0.00 & $2769 \pm 26$ & -0.06 & - \\
\hline
\end{tabular}

References for metallicity of primary companion: ${ }^{1}$ Bonfils et al. (2005), ${ }^{2}$ Valenti \& Fischer (2005). 

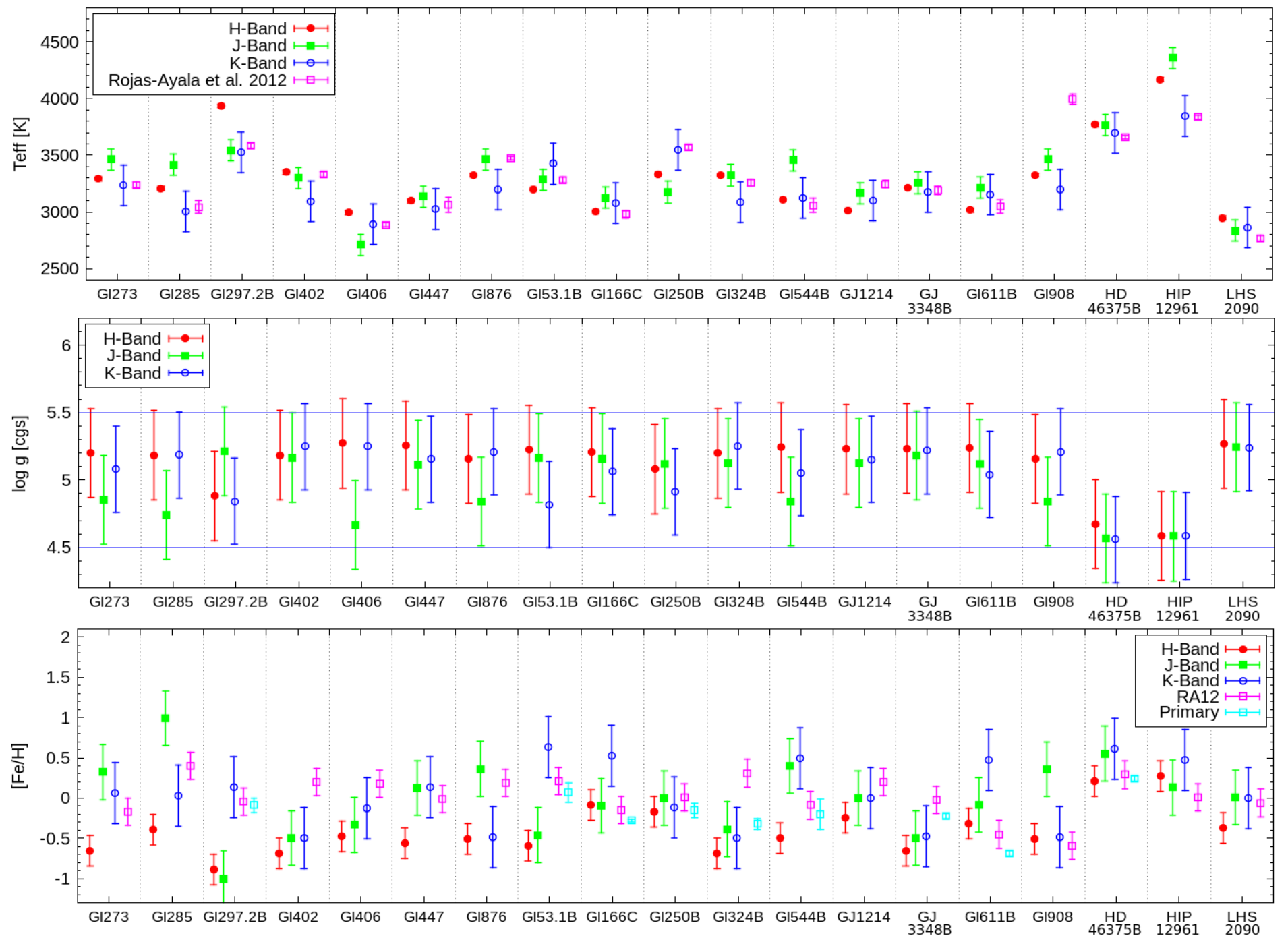

Figure 4.8.: Comparison of results for all TripleSpec spectra. For each star the values for temperature (top), surface gravity (middle) and metallicity (bottom) from J-, H-, and K-bands are plotted, together with the results from Rojas-Ayala et al. (2012). For stars that have an FGK companion the primary's metallicity is plotted from literature values. 


\subsection{Results from X-Shooter}

The main purpose of analysing X-Shooter spectra is to compare results from the NIR and VIS ranges. I present results from $13 \mathrm{X}$-Shooter spectra in the $\mathrm{H}$ - and J-bands as well as in the visible wavelength range. An example plot of the spectrum from Gl 285 with the best fit model is shown in Fig. 4.9 for the NIR and in Fig. 4.10 for the VIS. Table 4.2 shows $T_{\text {eff }}, \log g$ and $[\mathrm{Fe} / \mathrm{H}]$ for all the stars in each wavelength region together with results from Rojas-Ayala et al. (2012) for the stars we have in common. A graphical comparison of the values for each star is plotted in Fig. 4.11. As for the TripleSpec results the temperatures are mainly consistent except for some outliers. The log $g$ spread is slightly larger between the boundaries of 4.5 and 5.5 dex. The metallicities derived from the different wavelength bands have a large spread, except for Gl 285, Gl 297, Gl 393 and Gl 402, where all values are quite consistent. For Gl 105B and Gl 908, no metallicity values in the J-band are plotted since these are located out of range at -2.4 and -1.8 dex, respectively.
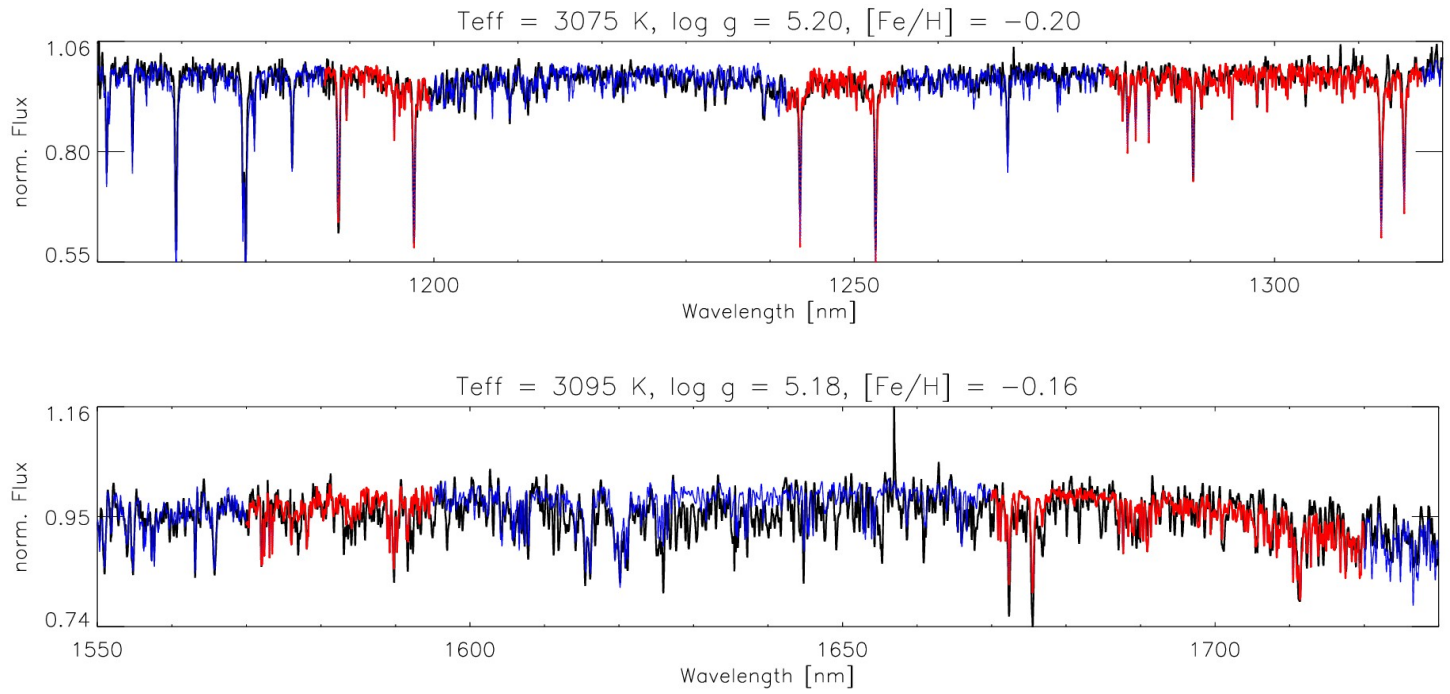

Figure 4.9.: Spectrum of G1 285 observed with X-Shooter (black) and the best fit model in blue. Regions used for $\chi^{2}$-minimisation are marked in red. Top panel: J-band; Bottom panel: H-band. 

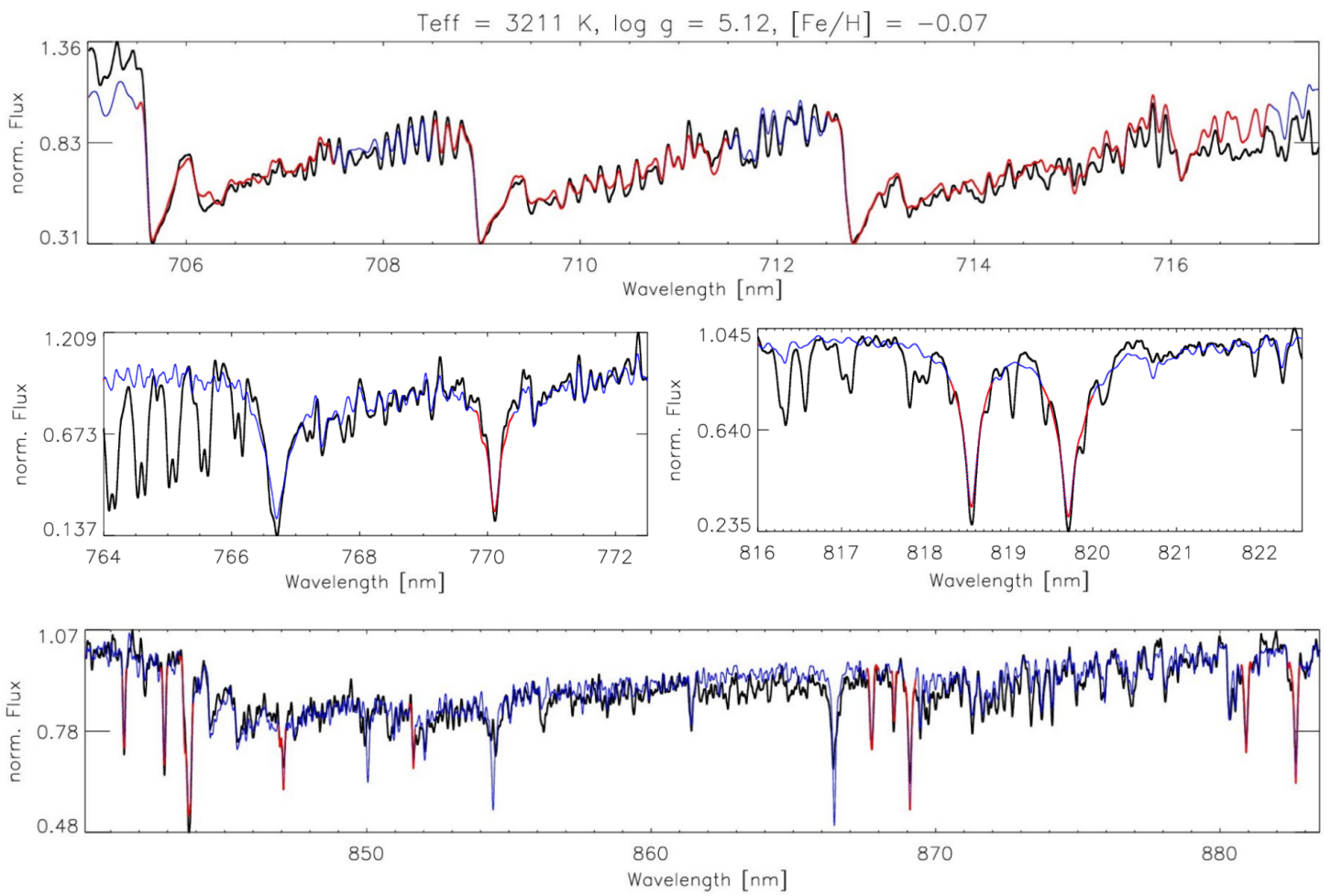

Figure 4.10.: Spectrum of G1 285 observed with X-Shooter (black) in the VIS and the best fit model in blue. Regions used for $\chi^{2}$-minimisation are marked in red. 
Table 4.2.: Results from X-Shooter spectra in the J-, H-bands and VIS, together with values from Rojas-Ayala et al. (2012) (RA12) for the stars we have in common.

\begin{tabular}{l|ccc|ccc|cccc|cc}
\hline \hline & \multicolumn{9}{|c}{$\mathrm{J}$} & \multicolumn{1}{c}{$\mathrm{H}$} & \multicolumn{3}{c}{$\mathrm{VIS}$} \\
\hline & $T_{\text {eff }}$ & $\log g$ & {$[\mathrm{Fe} / \mathrm{H}]$} & $T_{\text {eff }}$ & $\log g$ & {$[\mathrm{Fe} / \mathrm{H}]$} & $T_{\text {eff }}$ & $\log g$ & {$[\mathrm{Fe} / \mathrm{H}]$} & $T_{\text {eff }}$ & {$[\mathrm{Fe} / \mathrm{H}]$} \\
Name & \pm 107 & \pm 0.32 & \pm 0.29 & \pm 57 & \pm 0.33 & \pm 0.21 & \pm 121 & \pm 0.32 & \pm 0.33 & & \pm 0.17 \\
\hline Gl 105B & 3145 & 5.58 & -2.40 & 3388 & 5.17 & -0.68 & 3329 & 5.02 & 0.10 & & \\
Gl 166C & 3275 & 5.30 & -1.13 & 3015 & 5.18 & 0.01 & 3179 & 5.13 & -0.02 & $2979 \pm 32$ & -0.15 \\
Gl 273 & 3373 & 4.87 & 0.64 & 3143 & 5.15 & -0.08 & 3366 & 5.00 & 0.06 & $3239 \pm 26$ & -0.17 \\
Gl 285 & 3075 & 5.20 & -0.20 & 3095 & 5.19 & -0.16 & 3211 & 5.12 & -0.07 & $3045 \pm 59$ & 0.40 \\
Gl 297 & 3905 & 4.72 & -0.10 & 3745 & 4.90 & -0.47 & 3503 & 4.95 & -0.14 & $3585 \pm 20$ & -0.04 \\
Gl 328 & 4183 & 4.58 & 0.30 & 3900 & 4.50 & 0.83 & 4085 & 4.60 & 0.31 & & \\
Gl 393 & 3865 & 4.82 & -0.41 & 3371 & 5.11 & -0.38 & 3556 & 4.91 & -0.15 & & \\
Gl 402 & 3195 & 5.12 & -0.02 & 3179 & 5.18 & -0.30 & 3295 & 5.08 & -0.05 & $3334 \pm 23$ & 0.20 \\
Gl 406 & 2828 & 5.22 & 0.16 & 2311 & 4.43 & 0.99 & 3020 & 5.17 & 0.06 & $2887 \pm 20$ & 0.18 \\
Gl 447 & 3349 & 5.18 & -0.66 & 3066 & 5.23 & -0.34 & 3226 & 5.10 & -0.02 & $3065 \pm 69$ & -0.01 \\
Gl 678 & 3575 & 5.17 & -0.92 & 3676 & 4.73 & 0.13 & 3592 & 5.04 & -0.54 & & \\
Gl 908 & 4606 & 4.94 & -1.83 & 3475 & 4.99 & -0.22 & 3665 & 4.81 & -0.08 & $3995 \pm 47$ & -0.59 \\
Gl 2066 & 3893 & 4.68 & 0.08 & 3839 & 4.83 & -0.42 & 3534 & 4.92 & -0.11 & & \\
\hline
\end{tabular}



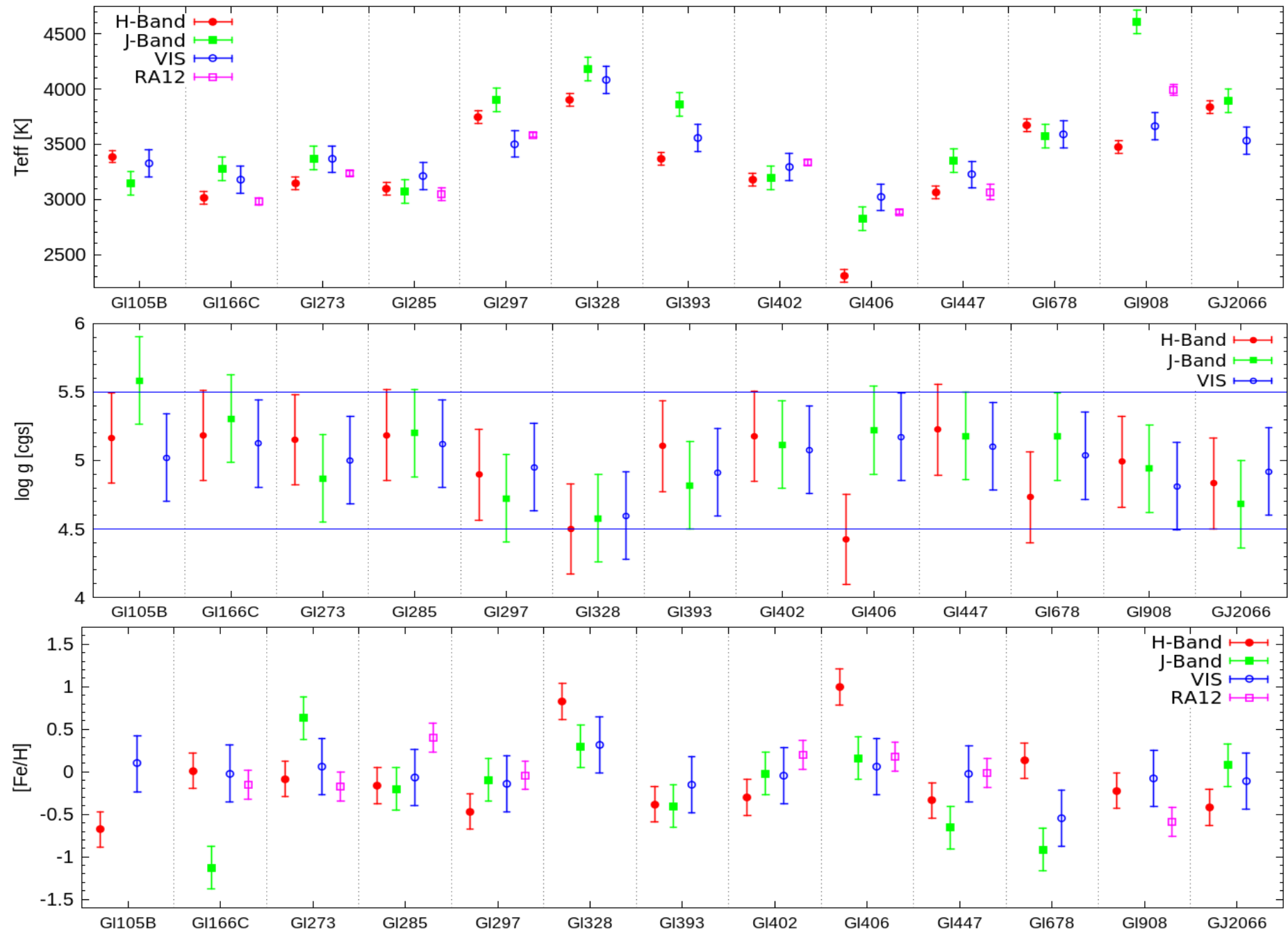

Figure 4.11.: Comparison of results for all X-Shooter spectra. For each star the values for temperature (top), surface gravity (middle) and metallicity (bottom) from J-, H-bands and the VIS are plotted, together with the results from Rojas-Ayala et al. (2012). 


\section{Discussion}

In this chapter I will discuss the obtained results from all spectrographs, present comparisons with literature values and show relations between spectral type, temperature and stellar mass. I will also provide possible explanations for outliers I found. In the end I will give a short overview on future work that has to be done to better understand the models and the data and to improve the results.

\subsection{CAFE, FEROS, HRS and CARMENES}

Comparison of CFH with CARMENES Since almost all stars observed with $C F H$ have also been observed with CARMENES, this offers the possibility to compare the obtained stellar parameters to investigate the stability of the method and possible dependencies on the different spectrographs and resolutions. The left column of Fig. 5.1 presents this comparison for effective temperature, $\log g$ and metallicity. The gray lines represent a $1 \sigma$ deviation of $93 \mathrm{~K}$ in temperature, 0.29 dex in $\log g$ and 0.25 dex in $[\mathrm{Fe} / \mathrm{H}]$. For the great majority, the CARMENES results confirm parameter values derived from CFH spectra. On average, temperatures from CAFE are systematically lower, but still well within the errors. A small offset can also be found in metallicity, where values from $\mathrm{CFH}$ spectra are systematically more metal-rich. There are a few outliers mainly from CAFE spectra, which might result from the poor SNR of some CAFE spectra compared to FEROS and CARMENES. These stars either show too high temperature and metallicity, or too low temperature and metallicity compared to the CARMENES parameters. This comparison as well illustrates that there seem to be no significant instrumental effects, like unknown light scattering within the optics or insufficient description of instrumental properties during the reduction process, which could cause inconsistencies in the resulting stellar parameters.

Another interesting exercise is the comparison of results from CARMENES template and single spectra. This can be seen on the right of Fig. 5.1. Again the gray lines represent a $1 \sigma$ deviation of $93 \mathrm{~K}, 0.29 \mathrm{dex}$ in $\log g$ and $0.25 \mathrm{dex}$ in metallicity. All results perfectly agree, which emphasises that for parameter determination the single spectra are sufficient and that the increased SNR of the template spectra has no advantageous effect here. However, one outlier can be found. For DS Leo, an M 2 star, the temperature resulting from the template spectrum is too high, compared to the spectral type. In addition, I also find a quite low $\log g$ and too high metallicity from the template. A possible explanation for the outliers found will be discussed in Section 5.2 . 

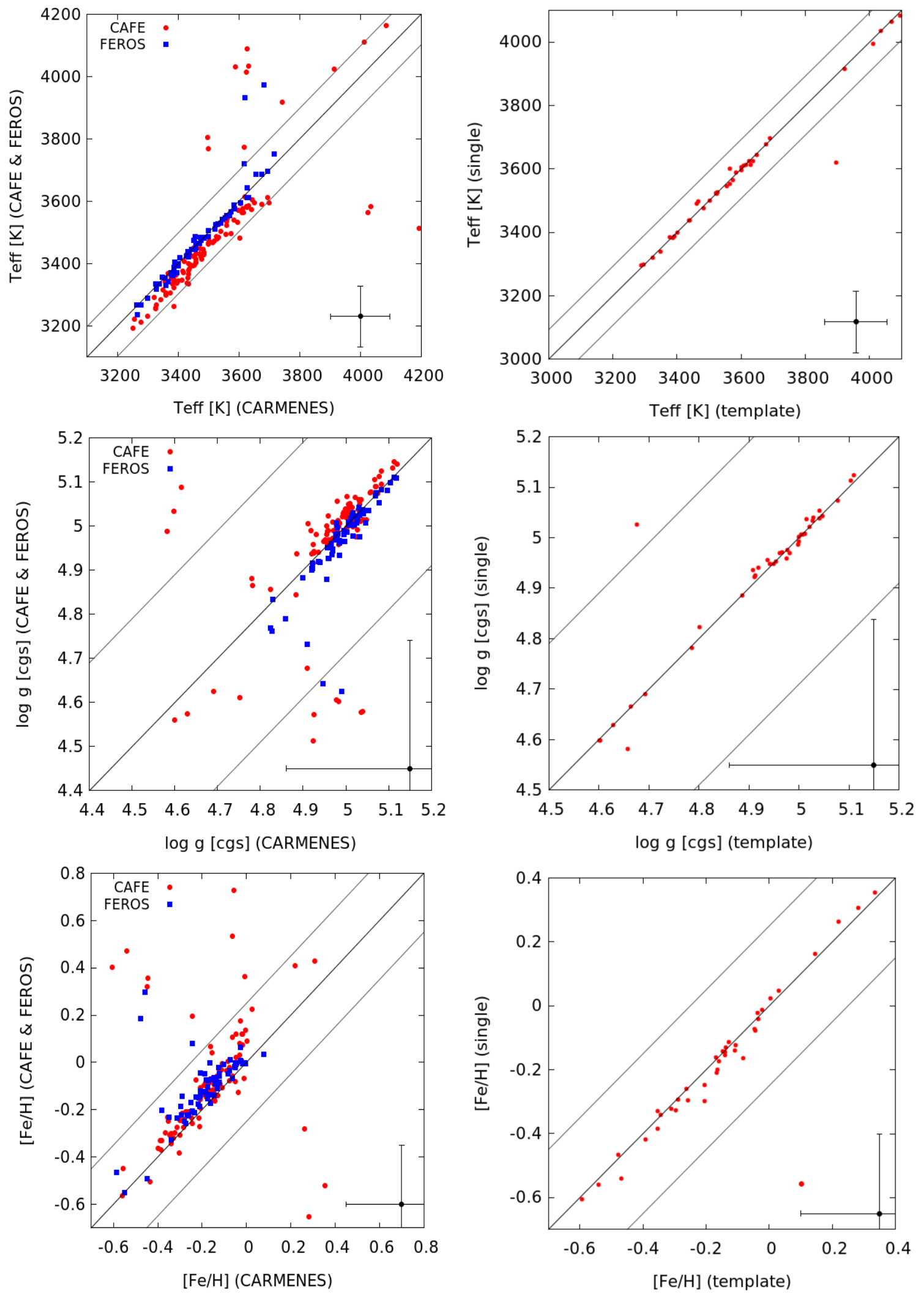

Figure 5.1.: Left: Comparison of results from $C F H$ spectra with results from CARMENES spectra. Right: Comparison of results from CARMENES template and single spectra. Top panels: effective temperature; Middle panel: surface gravity; Lower Panel: metallicity. 
Comparison with literature In Fig. 5.2 a comparison of those stars that overlap with the samples of Rojas-Ayala et al. (2012), Maldonado et al. (2015) and Gaidos \& Mann (2014) is presented. The gray lines indicate a $1 \sigma$ deviation of $93 \mathrm{~K}, 0.29$ dex in $\log g$ and 0.25 dex in $[\mathrm{Fe} / \mathrm{H}]$. Maldonado et al. (2015) estimated effective temperatures and metallicities using pseudo-equivalent widths in optical spectra. The upper panels show the results of effective temperatures. Although most of my results agree with literature within the errors, there are two groups of outliers on the cool and hot ends of the sample, which is present in the CFH sample (left) as well as in the CARMENES sample (right). The cool group is represented by results from Rojas-Ayala et al. (2012), derived from the $\mathrm{H}_{2} \mathrm{O}-\mathrm{K} 2$ index and calibrated with BT-Settl models of solar metallicity. The hot group is defined by results from optical spectra by Gaidos \& Mann (2014), who fitted BT-Settl models to obtain temperatures. They derive higher temperatures for stars hotter than $3500 \mathrm{~K}$.

In the middle panels of Fig. 5.2 the comparisons of surface gravity are plotted. Maldonado et al. (2015) derived $\log g$ using interferometrically determined masses and radii from early M dwarfs. Gaidos \& Mann (2014) provide stellar masses and radii calculated from their $T_{\text {eff }}$ using empirical relations from Mann et al. (2013b) and the massluminosity relation from Delfosse et al. (2000). I calculated $\log g$ for their sample with propagated errors from the uncertainties in mass and radius. My results are consistent with literature values within their errors, which is expected because I constrained $\log g$ using the $T_{\text {eff }}-\log g$ relations. This result also shows that the Baraffe et al. (1998) theoretical models agree very well with interferometric observations.

The bottom panels of Fig. 5.2 show the comparisons of metallicity. Again, most of the values coincide within their errors, however there are some outliers, especially in the upper left corner in the CARMENES sample plot. Compared to the other parameters, the spread in metallicity is slightly larger, which indicates that it is still difficult to constrain this parameter. The possibility that different methods can give different results must not be excluded as well. Although synthetic models for low-temperature stellar atmospheres improved significantly in the past decades, they still have some shortcomings. From Fig. 2.5 it can be seen that the cores of the K I line $(\lambda 770.1 \mathrm{~nm})$ and the $\mathrm{Na}$ I line pair $(\lambda 818.55 \mathrm{~nm}$ and $819.7 \mathrm{~nm})$ are not fully fitted. The core of these lines is formed in the stellar chromosphere and even up-to-date models are not able to account for this. In contrast, the line depths of e.g. Ti $\mathrm{I}(\lambda 846.9 \mathrm{~nm}$ and $867.77 \mathrm{~nm})$ and $\mathrm{Fe}$ I $(\lambda 867.71 \mathrm{~nm}$ and $882.6 \mathrm{~nm})$ are overestimated, which might result from problems in modelling the line-continuum contrast. Another possible explanation for the large spread will be discussed in the next section.

I compared the overall metallicity distribution of the $\mathrm{CFH}$ sample to the solar neighbourhood metallicity distribution from Holmberg et al. (2009). Within the GenevaCopenhagen survey they analysed more than 16,000 nearby F and G dwarfs, giving temperatures, metallicities, ages and accurate distances from Hipparcos parallaxes amongst other parameters. To compute the histogram shown in Fig. 5.3 I took metallicities from all stars within a distance of $40 \mathrm{pc}$ and an isochrone age between 4-6 Gyr (equivalent to the CARMENES stars). The two distributions are very similar to each other. As mentioned before, the CFH sample has a mean metallicity of $-0.11 \pm 0.20 \mathrm{dex}$, while the solar neighbourhood metallicity shows a mean of $-0.15 \pm 0.16$ dex. However, the high- 
metallicity tail in the CFH sample is not reflected in the solar neighbourhood. If this is real, which can be doubted from the literature comparison in Fig. 5.2, it would imply significant consequences on the formation and evolution of low-mass stars, at least in the solar neighbourhood. A more probable interpretation of these outliers is given in the next section.

Spectral Type - Temperature Fig. 5.4 presents effective temperature as a function of spectral type. It shows that the temperature increases with increasing spectral type as expected. Additionally, for the same spectral type, the temperature increases with increasing metallicity. In other words, for constant temperature, the metallicity increases towards later spectral types. Higher metallicity means more metals are present in the stellar atmosphere, which increases the opacity in the optical, due to $\mathrm{TiO}$ and VO molecules, but also decreases $\mathrm{H}_{2}$ opacity in the NIR K-band. Additionally, the peak of the energy distribution is shifted towards longer wavelengths, decreasing the flux in the optical and increasing it in the K-band. The star appears redder (i.e. of later spectral type) for the same temperature if the metal content is increased. These effects add up in the VIS, leading to a notable dependency between flux and metallicity. In the NIR K-band both effects almost cancel out, making K-band flux nearly independent to metallicity for stellar masses below $0.4 \mathrm{M}_{\odot}$. This again emphasises observations of $\mathrm{M}$ dwarfs in the NIR. Mann et al. (2015) found a similar trend, where metallicity and stellar radius increase for the same temperature (see their Figure 23). Maldonado et al. (2015) also presented in their Fig. 12 that for given temperature increasing metallicities predict slightly larger stellar masses and radii.

Mass - Temperature Fig. 5.5 presents the relation between stellar mass and effective temperature, with the metallicities colour-coded. The theoretical relation from Baraffe et al. (1998) is shown by the green line for an age of $5 \mathrm{Gyr}$ and solar metallicity. The masses were taken from the Carmencita database. They have been calculated from $\mathrm{m}_{k}$ magnitudes using the mass-luminosity relation from Delfosse et al. (2000). Note that no errors are provided from this relation. A slight spread in metallicity is found here as well, with sub-solar metallicities lying above the green curve and super-solar metallicities below. This behaviour was also found by Gaidos \& Mann (2014). Their Figure 5 illustrates stellar masses calculated from $\mathrm{m}_{k}$ magnitudes and the Delfosse et al. (2000) mass-luminosity relation as a function of stellar masses determined from $T_{\text {eff }}$. The same metallicity dependent trend can be seen. It again shows that metallicity has a considerable effect on the atmospheric structure. Detailed studies on that can be found by Brett (1995) and Allard \& Hauschildt (1995). Since for lower metallicity the mean opacity of the stellar atmosphere decreases, the atmosphere gets more transparent. The same optical depths then lie in deeper layers of the atmosphere at higher local temperatures. The luminosity therefore increases because energy can escape more easily, which means that for the same mass effective temperature increases with decreasing metallicity.

Some outliers with sub-solar metallicities can be found between 0.5 and $0.6 \mathrm{M}_{\odot}$ and around $3500 \mathrm{~K}$, although their temperatures estimated from spectral type should lie between $3800-3900 \mathrm{~K}$. The next section gives a possible explanation for this discrepancy. 

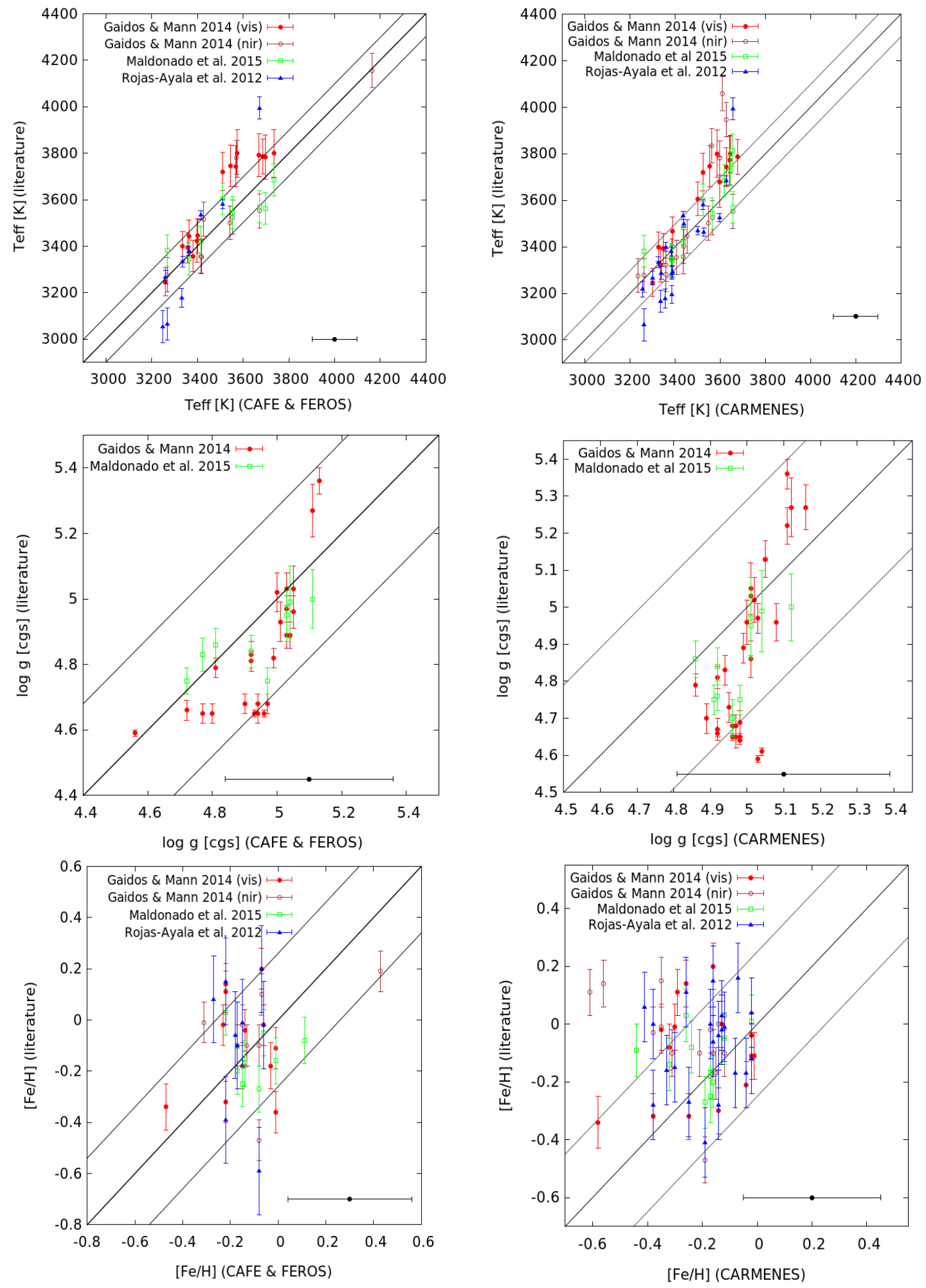

Figure 5.2.: Comparison of results from $C F H$ and CARMENES spectra with literature. Top panel: effective temperature; Middle panel: surface gravity; Lower Panel: metallicity. 


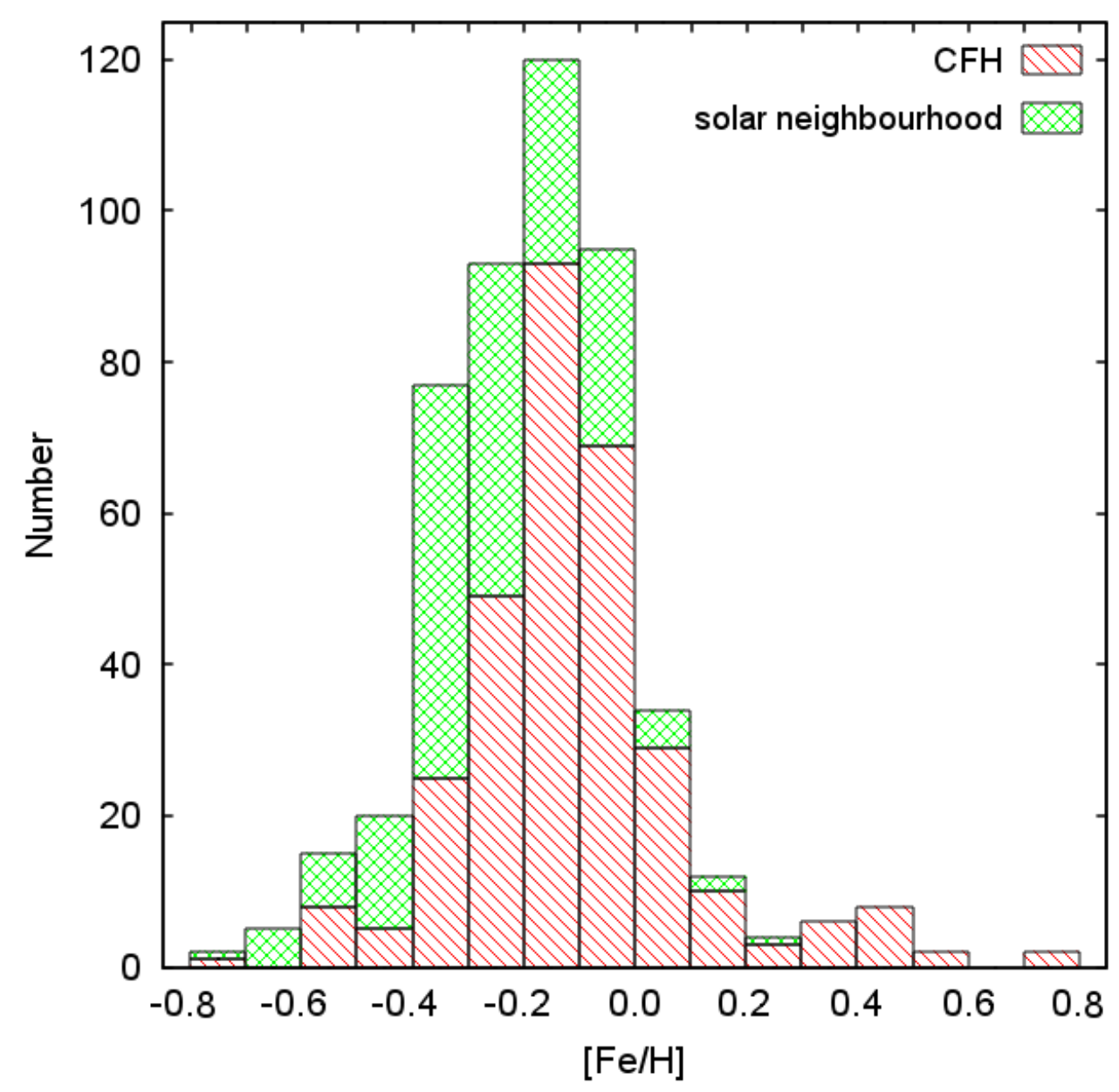

Figure 5.3.: Comparison of the overall metallicity distribution of the $\mathrm{CFH}$ sample (red) with the distribution of the solar neighbourhood (green). 


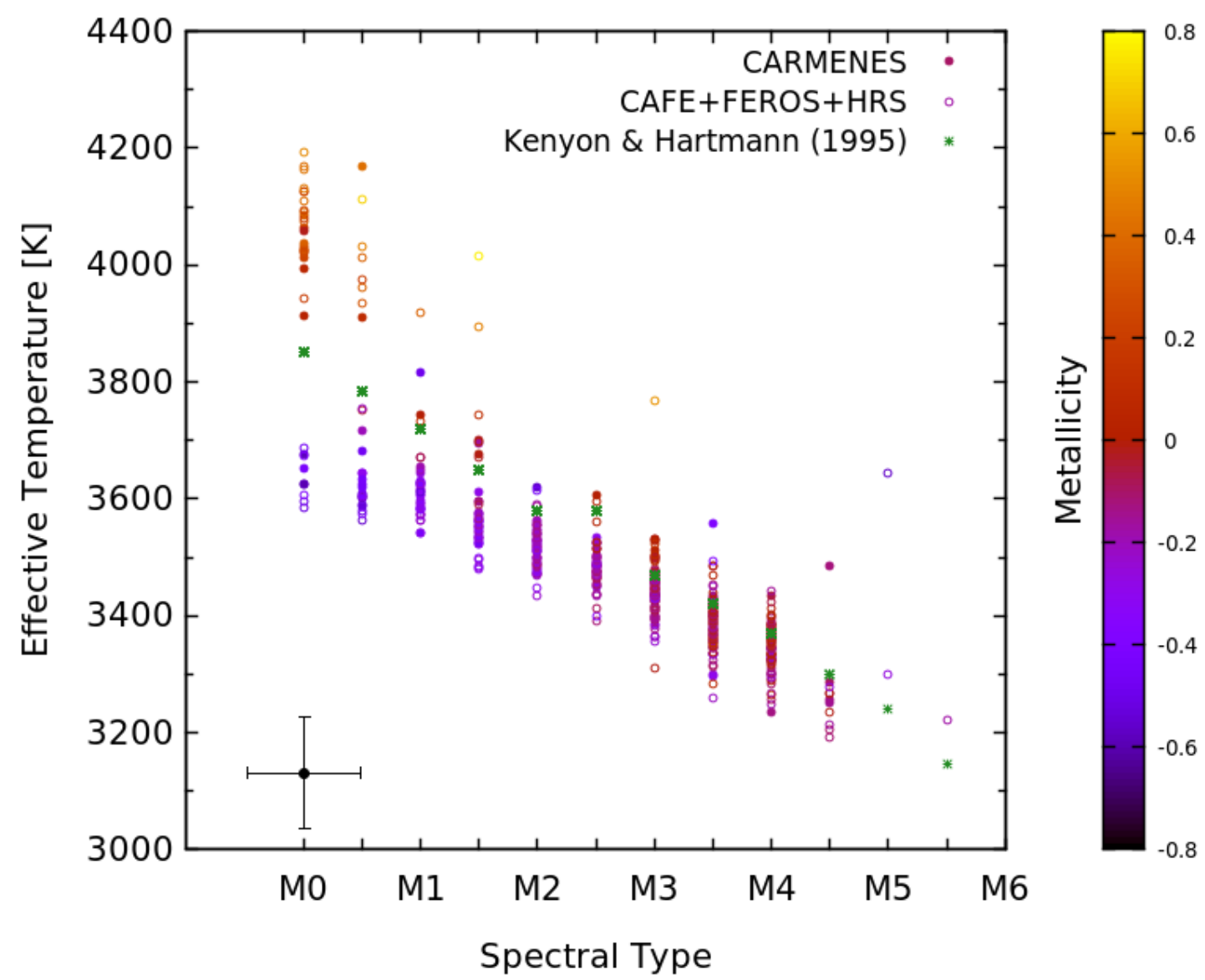

Figure 5.4.: Effective temperature as a function of spectral type for the stars from $\mathrm{CFH}$ (left) and CARMENES (right). Metallicity is colour-coded on the right side. The green star-shaped symbols represent literature values from Kenyon \& Hartmann (1995) for solar metallicity. 


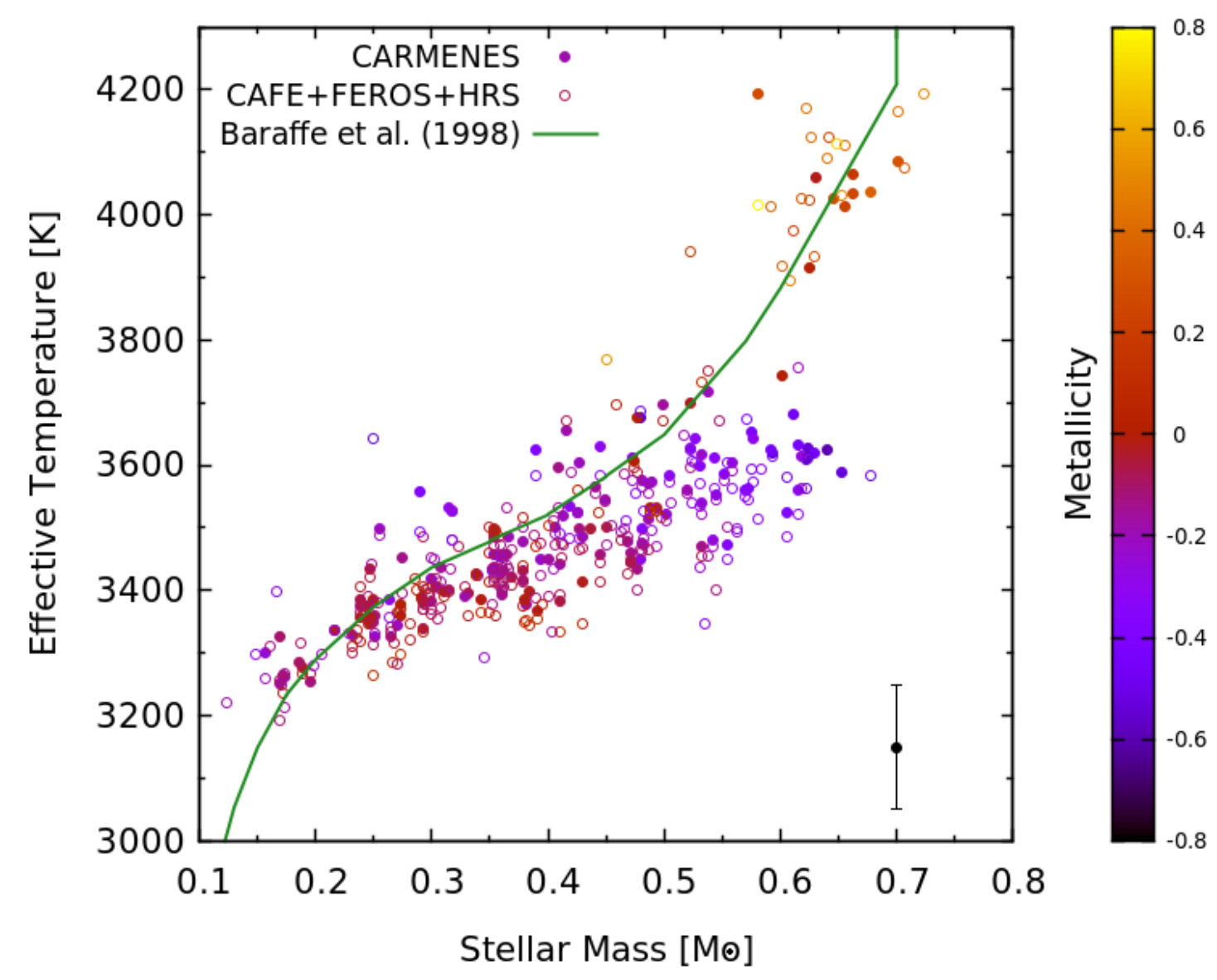

Figure 5.5.: Effective temperature as a function of stellar mass for the stars from $C F H$ and CARMENES. Metallicity is colour-coded on the right side. The green line represents the theoretical relation from Baraffe et al. (1998) evolutionary models for solar metallicity and an age of 5 Gyr. 


\subsection{Comparison of model atmospheres}

As mentioned in the last section, I find some outliers, especially in temperature and metallicity. When looking at the mass-temperature plot of Fig. 5.5, I derive too low temperatures for the group of outliers on the right below the theoretical relation from Baraffe et al. (1998). All these stars have spectral types earlier than $\sim \mathrm{M} 1.5 \mathrm{~V}$, which means the expected temperatures lie around $3900 \mathrm{~K}$ instead of $3500 \mathrm{~K}$. This leads to the hypothesis that there could be 2 sets of parameters, namely low $T_{\text {eff }}$ and $[\mathrm{Fe} / \mathrm{H}]$ and high $T_{\text {eff }}$ and $[\mathrm{Fe} / \mathrm{H}]$, where models look very similar. To support my theory I assume a reference model spectrum with parameters $4000 \mathrm{~K}, 5.0 \log g$ and $+0.5[\mathrm{Fe} / \mathrm{H}]$. With $\log g$ fixed I then compare models with different temperatures $(3000-4000 \mathrm{~K}$, step size $100 \mathrm{~K})$ and metallicities $(-1.0,-0.5,0.0,+0.5$ and $+1.0 \mathrm{dex})$ to the reference model and calculate the deviations for the lines I use for fitting (see Section 2.3). The result of that test is presented in Fig. 5.6. Whereas lines/regions like the $\gamma$-TiO band, Ti-, Fe- and Mglines show a similar pattern, the behaviour of the $\mathrm{K}$ - and Na-lines proves the hypothesis. Deviations minimise for $T_{\text {eff }} \sim 3600 \mathrm{~K}$ almost independent of metallicity. Since I apply higher weights to these lines due to their sensitivity to metallicity, the algorithm might find a local minimum here and prefers the solution for lower temperature and sub-solar metallicity for hot $\mathrm{M}$ dwarfs (i.e. with spectral types earlier than $\sim \mathrm{M} 1.5 \mathrm{~V}$ ). It cannot be excluded that this also happens the other way around, meaning a hot and metal-rich model is preferred for a cooler star.

Fig. 5.7 presents a CARMENES spectrum of the M0.0 star V2689 Ori. For this star, the algorithm found two parameter sets with low $\chi^{2}$, a hot one $\left(T_{\text {eff }}=4081 \mathrm{~K}, \log g=4.62\right.$, $[\mathrm{Fe} / \mathrm{H}]=+0.18$, plotted in blue and red $)$ and a cool one $\left(T_{\text {eff }}=3675 \mathrm{~K}, \log g=5.04,[\mathrm{Fe} / \mathrm{H}]=-\right.$ 0.7 , plotted in purple and green), the former being preferred by a slightly smaller $\chi^{2}$. The deviation between the two models is also plotted for each wavelength range. The plot illustrates that the two models have deviations of more than $25 \%$ in the TiO bands, similar to the Mg-line with up to $20 \%$. As stated before, the two models deviate only slightly from each other in the Na-line pair. While the cores are identical, the wings differ by less than 5\%. The same deviations applies to the Ti-lines between 841 and $844 \mathrm{~nm}$. This analysis shows that the $\mathrm{K}$ - and Na-lines seem to be not as suitable for metallicity determination as stated in Section 2.3. Additionally, the models are having problems to reproduce the depth of the Na-lines, since these lines are formed in or near the chromosphere for active stars like V2689 Ori, which can also be seen in Fig. 5.7. However, the wings can still be used.

This finding can explain almost all outliers, e.g. the hot group of Gaidos \& Mann (2014) in the upper panel of Fig. 5.2, the large spread in metallicity in the lower panel of the same figure, the different results for DS Leo and the outliers in the $C F H$ and CARMENES comparison.

A similar discrepancy was not found by other authors before. For optical spectra Gaidos \& Mann (2014) fitted temperatures from BT-Settl models, but metallicities are determined from the NIR. Mann et al. (2015) used a similar approach, deriving temperatures from BT-Settl model fits and metallicities from empirical relations of equivalent width in the NIR. The work of Rajpurohit et al. (2014) is more similar to mine, they also used high-resolution spectra and determined stellar parameters from interpolating model 
spectra and $\chi^{2}$-minimisation. But they fitted BT-Settl models to a small sample of 18 $\mathrm{M}$ dwarfs. This work is the first to derive $T_{\text {eff }}, \log g$ and $[\mathrm{Fe} / \mathrm{H}]$ from fitting PHOENIXACES models to a large sample of high-resolution spectra, which also allows to reveal inconsistencies in the models.

A study similar to mine was done by Lindgren et al. (2016). They determined metallicities of 8 single $\mathrm{M}$ dwarfs and $4 \mathrm{FGK}+\mathrm{M}$ systems from high-resolution infrared spectra. They used MARCS models (Gustafsson et al., 2008) together with the Spectroscopy Made Easy (SME) package (Valenti \& Piskunov, 1996) and find the best fit using interpolation between the model grid points and $\chi^{2}$-minimisation. Other than for PHOENIX, the solar abundances come from Grevesse et al. (2007). Effective temperatures are estimated from $\mathrm{FeH}$ lines, the metallicities result from fits to all atomic species (except for C-lines due to possible non-LTE effect in the FGK companions). As presented in the contour plots of their Fig. 2, the FeH line strength shows a strong dependency on temperature, but only a weak dependency on metallicity, which leads to a large degeneracy between these two parameters. On the other hand, the metallicities derived from the hot and cool companion of the FGK $+\mathrm{M}$ systems agree with each other and confirm the validity of their method. However, the study shows that other synthetic models as well suffer from degeneracies. 

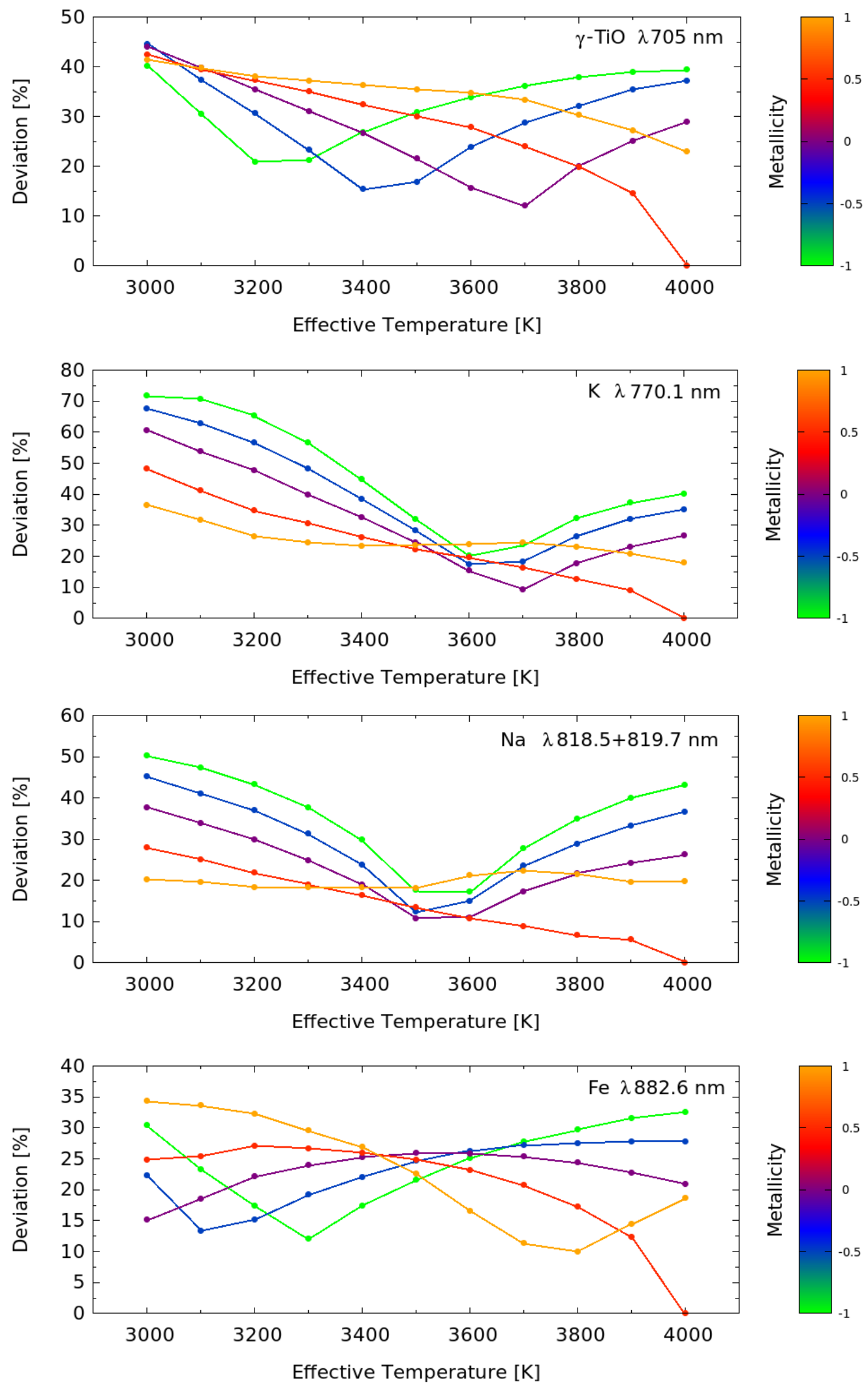

Figure 5.6.: Deviations of model spectra with different parameters to the reference model as a function of effective temperature. Metallicity is colour-coded on the right side. A selection of analysed lines/regions is presented because the $\gamma$-TiO band, Ti-, Feand $\mathrm{Mg}$-lines show a similar behaviour. 

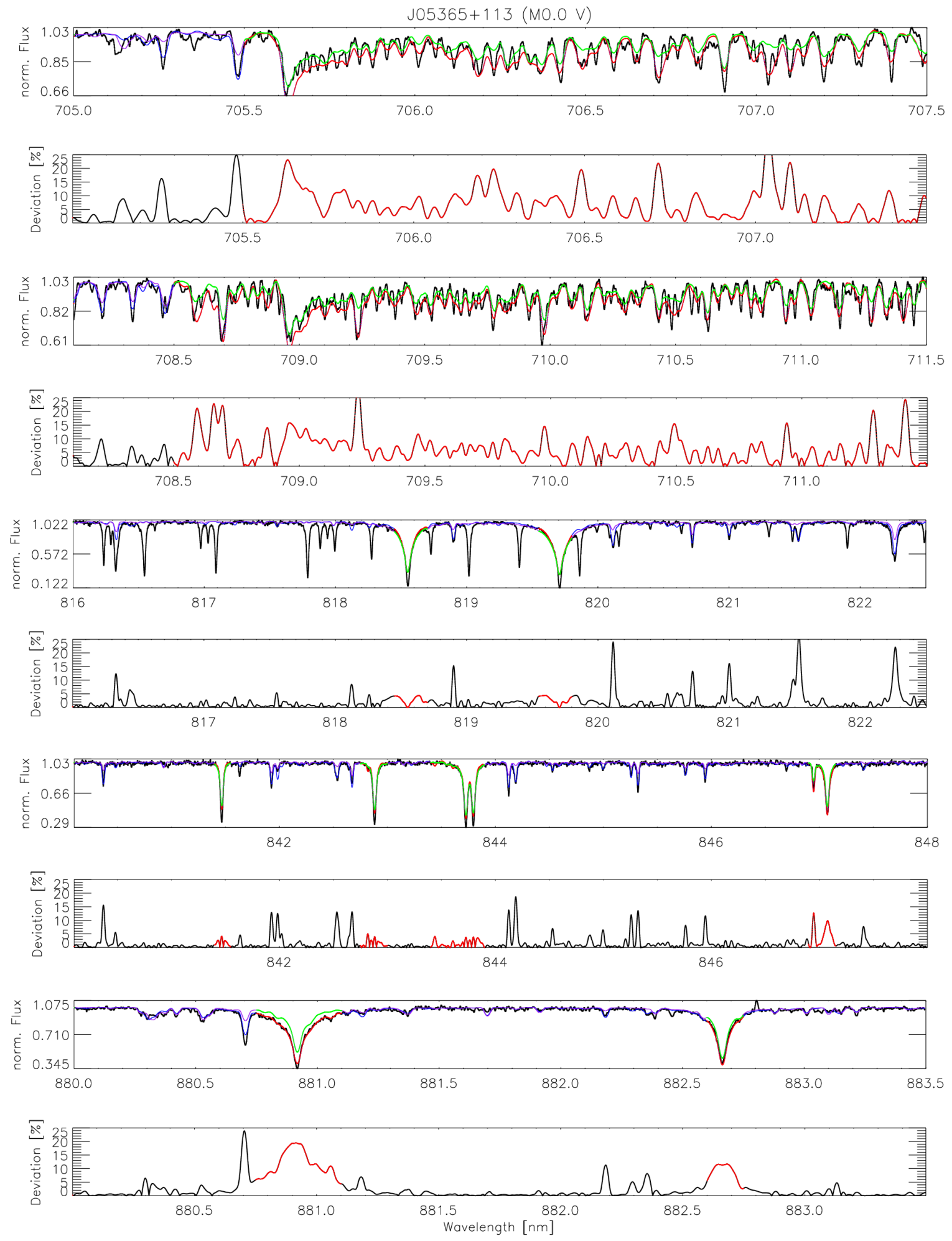

Figure 5.7.: CARMENES spectrum of V2689 Ori (black) with two PHOENIXACES models; The blue/red lines present the hot model $\left(T_{\text {eff }}=4081 \mathrm{~K}, \log g=4.62\right.$, $[\mathrm{Fe} / \mathrm{H}]=+0.18)$ and the purple/green lines show the cool model $\left(T_{\text {eff }}=3675 \mathrm{~K}\right.$, $\log g=5.04,[\mathrm{Fe} / \mathrm{H}]=-0.7)$. As in the previous plots, the green and red parts present the regions used for $\chi^{2}$ calculation. Below each wavelength range the deviation between the hot and the cool model is shown. The red regions illustrate again the lines used for $\chi^{2}$ calculation. Only a selection of wavelength ranges is presented. 


\subsection{TripleSpec and X-Shooter}

TripleSpec Comparing results from different NIR bands shows that temperature and $\log g$ values mostly agree with each other within their errors. Temperature results from Rojas-Ayala et al. (2012) are also mostly consistent with my values. Their measurements, derived from the K-band, coincide with my values determined from the K-band for 15 of 19 stars. For metallicities there is a large spread and there is no clear trend visible. This could be because of the different determination method of Rojas-Ayala et al. (2012), but further emphasises that there are some difficulties in the determination of metallicities in the NIR and from different NIR bands. Moreover, metallicities derived from the $\mathrm{H}$-band are tentatively lower than values from other wavelength regions. This might be explained by the low number of lines suitable for fitting in that region, compared to the J- and K-bands, which can be seen in Fig. 4.7. For stars with an FGK primary companion, the derived metallicities generally match with my results within their errors, which on the other hand underlines the robustness of my method.

X-Shooter For temperature most of my measurements in the different wavelength bands agree with each other within their errors, except for Gl 406 and Gl 908. The $\log g$ values are widely consistent as well. For Gl 328, all three measurements lie on the lower limit of $4.5 \mathrm{dex}$. The temperature yields around $4000 \mathrm{~K}$, which matches the spectral type M 0.0 stated by Lépine et al. (2013) and can therefore support the derived $\log g$. As for the TripleSpec results, the spread in metallicity is larger, again without any obvious trends. Serious outliers are Gl 105B, Gl 406 and Gl 908. Since Gl 406 is highly magnetically active, this could be an explanation for the deviations. Similar differences are also found in the TripleSpec results. The other two stars are not known to be considerably active, however for both of them the deviations are found in the J-band. For these spectra there are still large telluric remnants in this band.

In general, the telluric correction of X-Shooter spectra could be improved, since there are still many telluric remnants in the cleaned spectra which might influence the parameter determination. However, this first study of NIR and VIS spectra from the same spectrograph illustrates that most derived parameters match and motivates the upcoming analysis of CARMENES NIR and VIS spectra.

\subsection{Future work}

As mentioned in the previous sections of this chapter, some work still has to be done to completely understand inconsistencies, especially concerning treatment of different lines in synthetic model atmospheres. The short comparison in Section 5.2 already showed that there are sets of parameters for which the models can look very similar in certain lines. That degeneracy could be broken by adjusting weights, in order to get accurate parameters also for hot $\mathrm{M}$ dwarfs. This requires a careful analysis of the models, comparing lines for different parameter sets and weights. A correct adjustment might also help to accurately determine $\log g$ from model fits instead of evolutionary models. Later 
on, an adaption of the models themselves could become necessary. The previous fits already showed that for some lines would need improvement, e.g. the K-band Ca triplet. Since CARMENES offers the possibility to observe stars simultaneously in the VIS and NIR, parameter determination in both ranges is desirable. A comparison of both results could help to improve models and understand any unknown wavelength dependent effects on stellar parameters. For this purpose the sensitivity of lines in the NIR has to be confirmed by more detailed studies. From the analysis of the VIS spectra we saw how crucial a careful and detailed study of potentially sensitive lines is. Because the CARMENES spectra have much higher resolution than spectra from TripleSpec and XShooter, more spectral details are revealed, which makes a careful study necessary. This can be done by comparing the change in line strength and width for models with different parameter sets. Lines that show large changes are potentially suitable for fitting. However, they need to be checked in stars with known parameters to see if the models recover the line shape correctly.

The Gaia mission, launched in December 2013, provides highly accurate positions of about 1 billion stars. The CARMENES project can also benefit from these measurements by calculating stellar masses and radii from the Gaia distances. This information is fundamental for further planet characterisation. 


\section{Summary}

In the past years, planet search projects focused more and more on $\mathrm{M}$ dwarfs. Transit searches benefit from the smaller radius ratio between star and planet and the smaller orbital periods for planets in the habitable zone. Radial-velocity searches, on the other hand, benefit from the smaller mass ratio and therefore higher radial-velocity amplitude. The CARMENES instrument is a next-generation instrument located at the $3.5 \mathrm{~m}$ telescope at Calar Alto Observatory in Spain. It consists of two high-resolution $(R \sim 82000)$ spectrographs operating in the visible $(0.55-0.95 \mu \mathrm{m})$ and near-infrared $(0.95-1.7 \mu \mathrm{m})$ wavelength range with precisions of around $1 \mathrm{~ms}^{-1}$. To characterise the target sample, more than 1700 spectra have been observed with other high-resolution spectrographs like CAFE, FEROS and HRS prior to the start of the CARMENES survey. For the determination of fundamental stellar parameters, i.e. effective temperature $\left(T_{\text {eff }}\right)$, surface gravity $(\log g)$ and metallicity [Fe/H], I developed an algorithm to fit up-to-date synthetic models to the observations. The new PHOENIX-ACES model grid especially accounts for the formation of molecules in cool stellar atmospheres. My algorithm determines the best fit using linear interpolation of the model grid, a downhill-simplex method and $\chi^{2}$-minimisation. With this I analyse different samples of stars, the preparation sample observed with CAFE, FEROS and HRS (1390 spectra, 484 stars), the first six month of CARMENES data (1738 spectra, 245 stars) and near-infrared spectra taken with TripleSpec (19 spectra/stars) and X-Shooter (13 spectra/stars). I take into account $v$ sin is measured by Jeffers et al. (submitted) from cross-correlation. To reduce the number of free parameters in the fit and get more accurate results, $\log g$ is determined from evolutionary models by Baraffe et al. (1998) depending on $T_{\text {eff }}$ and $[\mathrm{Fe} / \mathrm{H}]$. I obtain good results for $323 \mathrm{M}$ dwarfs.

A comparison between results of the same stars from spectra obtained by different spectrographs mainly shows agreement. Literature values are also generally consistent with my results, however there are a few outliers. Some of these outliers can be statistically expected, also because of the different quality and SNR of the spectra. They might as well be a hint for still existing inconsistencies in synthetic models or the use of different determination methods, especially regarding metallicities. A quite promising explanation is supported by the finding that there are (at least) two different sets of parameters for which the PHOENIX-ACES models look very similar in some spectral regions, namely high temperature and metallicity ( 3 900-4000 K, super-solar metallicity) and low temperature and metallicity ( 3 500-3600 K, sub-solar metallicity). It especially concerns the K- and Na-lines. With higher weighting of these lines due to their sensitivity in metallicity, the algorithm might fall into a local minimum and prefer a cooler temperature solution for hot $\mathrm{M}$ dwarfs (i.e. earlier than $\sim \mathrm{M} 1.5 \mathrm{~V}$ ). A more detailed investigation of this behaviour is needed to find proper weights that yield precise results for all spectral types. 
This is the first study that derives $T_{\text {eff }}, \log g$ and $[\mathrm{Fe} / \mathrm{H}]$ from fitting PHOENIX-ACES models to high-resolution optical spectra. It shows that accurate determination of stellar parameters of a large sample of $\mathrm{M}$ dwarfs is possible using an automated algorithm. With the fiasco-code I obtain uncertainties of $93 \mathrm{~K}, 0.29 \mathrm{dex}$ in $\log g$ and $0.25 \mathrm{dex}$ in $[\mathrm{Fe} / \mathrm{H}]$. However, a lot of detailed analysis is still necessary to completely understand the behaviour of spectral lines with changing parameters, and of synthetic models in general. 


\section{A. Appendix: Tables with stellar parameters}


Table A.1.: Measured astrophysical parameters of investigated stars. The table lists $\mathrm{Car}$ mencita identifier (Karmn), spectral type, effective temperature, surface gravity, metallicity, rotational velocity ${ }^{a}$, Ca II emission flag, and instrument with which the spectrum was obtained (templ. standing for CARMENES template).

\begin{tabular}{|c|c|c|c|c|c|c|c|}
\hline Karmn & $\begin{array}{l}\text { Spectral } \\
\text { type }\end{array}$ & $\begin{array}{c}T_{\text {eff }} \\
{[\mathrm{K}]} \\
( \pm 93)\end{array}$ & $\begin{array}{c}\log g \\
{[\mathrm{cgs}]} \\
( \pm 0.29)\end{array}$ & $\begin{array}{c}{[\mathrm{Fe} / \mathrm{H}]} \\
{[\mathrm{dex}]} \\
( \pm 0.25)\end{array}$ & $\begin{array}{c}v \sin i^{a} \\
{\left[\mathrm{kms}^{-1}\right]}\end{array}$ & $\begin{array}{l}\text { Ca II } \\
\text { emis. }\end{array}$ & Instr. \\
\hline \multirow{2}{*}{$\mathrm{J} 00051+457$} & $\mathrm{M} 1.0 \mathrm{~V}$ & 3572 & 4.96 & -0.31 & $<3$ & $\ldots$ & CAFE \\
\hline & & 3598 & 4.96 & -0.35 & $<3$ & $\ldots$ & CARM. \\
\hline $\mathrm{J} 00056+458$ & M0.0 V & 4094 & 4.61 & +0.23 & $<3$ & $\ldots$ & CAFE \\
\hline $\mathrm{J} 00162+198 \mathrm{E}$ & M4.0 V & 3346 & 5.04 & -0.02 & $<3$ & $\ldots$ & FEROS \\
\hline $\mathrm{J} 00162+198 \mathrm{~W}$ & $\mathrm{M} 4.0 \mathrm{~V}$ & 3395 & 5.02 & -0.10 & 3.6 & Yes & FEROS \\
\hline \multirow[t]{2}{*}{$\mathrm{J} 00183+440$} & M1.0 V & 3606 & 4.95 & -0.32 & $2.5^{b}$ & $\ldots$ & CARM. \\
\hline & & 3599 & 4.94 & -0.30 & $2.5^{b}$ & $\ldots$ & templ. \\
\hline \multirow[t]{2}{*}{ J00184+440 } & $\mathrm{M} 3.5 \mathrm{~V}$ & 3297 & 5.12 & -0.30 & $1.9^{c}$ & $\ldots$ & CARM. \\
\hline & & 3288 & 5.11 & -0.20 & $1.9^{c}$ & $\ldots$ & templ. \\
\hline J00286-066 & $\mathrm{M} 4.0 \mathrm{~V}$ & 3413 & 4.99 & -0.01 & $<3$ & $\ldots$ & FEROS \\
\hline J00315-058 & M3.5 V & 3394 & 5.03 & -0.12 & $<3$ & $\ldots$ & FEROS \\
\hline $\mathrm{J} 00395+149 \mathrm{~S}$ & $\mathrm{M} 4.0 \mathrm{~V}$ & 3354 & 5.06 & -0.12 & $<3$ & $\ldots$ & HRS \\
\hline $\mathrm{J} 00443+126$ & $\mathrm{M} 3.5 \mathrm{~V}$ & 3418 & 4.98 & +0.00 & $<3$ & $\ldots$ & FEROS \\
\hline $\mathrm{J} 00566+174$ & $\mathrm{M} 4.0 \mathrm{~V}$ & 3316 & 5.06 & -0.05 & $<3$ & $\ldots$ & FEROS \\
\hline $\mathrm{J} 00570+450$ & M3.0 V & 3410 & 5.01 & -0.09 & $<3$ & $\ldots$ & CAFE \\
\hline J01009-044 & $\mathrm{M} 4.0 \mathrm{~V}$ & 3325 & 5.07 & -0.12 & $<3$ & $\ldots$ & FEROS \\
\hline $\mathrm{J} 01013+613$ & $\mathrm{M} 2.0 \mathrm{~V}$ & 3522 & 4.96 & -0.23 & $4.0^{d}$ & $\ldots$ & CARM. \\
\hline $\mathrm{J} 01025+716$ & $\mathrm{M} 3.0 \mathrm{~V}$ & 3512 & 4.90 & -0.01 & $2.5^{b}$ & $\ldots$ & CARM. \\
\hline \multirow[t]{2}{*}{$\mathrm{J} 01026+623$} & M1.5 V & 3545 & 4.96 & -0.25 & $<3$ & $\ldots$ & CAFE \\
\hline & & 3563 & 4.98 & -0.35 & $<3$ & Yes & CARM. \\
\hline $\mathrm{J} 01256+097$ & $\mathrm{M} 4.0 \mathrm{~V}$ & 3328 & 5.07 & -0.12 & $<3$ & $\ldots$ & FEROS \\
\hline $\mathrm{J} 01384+006$ & $\mathrm{M} 2.0 \mathrm{~V}$ & 3541 & 4.95 & -0.22 & $<3$ & $\ldots$ & FEROS \\
\hline $\mathrm{J} 01433+043$ & $\mathrm{M} 2.0 \mathrm{~V}$ & 3515 & 4.95 & -0.17 & $2.5^{b}$ & $\ldots$ & CARM. \\
\hline J01466-086 & $\mathrm{M} 4.0 \mathrm{~V}$ & 3400 & 5.00 & +0.00 & $<3$ & $\ldots$ & FEROS \\
\hline J02050-176 & $\mathrm{M} 2.5 \mathrm{~V}$ & 3485 & 4.97 & -0.15 & $<3$ & $\ldots$ & FEROS \\
\hline $\mathrm{J} 02070+496$ & $\mathrm{M} 3.5 \mathrm{~V}$ & 3389 & 5.04 & -0.14 & $<3$ & $\ldots$ & CAFE \\
\hline J02096-143 & $\mathrm{M} 2.5 \mathrm{~V}$ & 3470 & 5.00 & -0.21 & $<3$ & $\ldots$ & FEROS \\
\hline $\mathrm{J} 02116+185$ & M3.0 V & 3460 & 4.95 & -0.02 & $<3$ & $\ldots$ & FEROS \\
\hline $\mathrm{J} 02123+035$ & M1.5 V & 3697 & 4.77 & -0.01 & $<3$ & $\ldots$ & FEROS \\
\hline $\mathrm{J} 02129+000$ & M4.0 V & 3310 & 5.08 & -0.13 & $<3$ & $\ldots$ & FEROS \\
\hline \multirow[t]{3}{*}{ J02222+478 } & M0.5 V & 3563 & 5.06 & -0.56 & 4.0 & $\ldots$ & CAFE \\
\hline & & 3610 & 5.03 & -0.56 & 4.0 & $\ldots$ & CARM. \\
\hline & & 3609 & 5.03 & -0.54 & 4.0 & $\ldots$ & templ. \\
\hline $\mathrm{J} 02336+249$ & $\mathrm{M} 4.0 \mathrm{~V}$ & 3299 & 5.11 & -0.21 & 3.1 & $\ldots$ & FEROS \\
\hline $\mathrm{J} 02358+202$ & $\mathrm{M} 2.0 \mathrm{~V}$ & 3503 & 4.99 & -0.26 & $<3$ & $\ldots$ & CAFE \\
\hline
\end{tabular}


Table A.1.: Measured astrophysical parameters of investigated stars (cont.).

\begin{tabular}{|c|c|c|c|c|c|c|c|}
\hline Karmn & $\begin{array}{l}\text { Spectral } \\
\text { type }\end{array}$ & $\begin{array}{c}T_{\text {eff }}[\mathrm{K}] \\
( \pm 93)\end{array}$ & $\begin{array}{c}\log g[\mathrm{cgs}] \\
( \pm 0.29)\end{array}$ & $\begin{array}{c}{[\mathrm{Fe} / \mathrm{H}]} \\
( \pm 0.25)\end{array}$ & $\begin{array}{c}v \sin i^{a} \\
{\left[\mathrm{kms}^{-1}\right]}\end{array}$ & $\begin{array}{l}\text { Ca II } \\
\text { emis. }\end{array}$ & Instr. \\
\hline & & 3540 & 4.96 & -0.24 & $<3$ & $\ldots$ & CARM. \\
\hline & & 3529 & 4.95 & -0.20 & $<3$ & $\ldots$ & FEROS \\
\hline \multirow[t]{2}{*}{$\mathrm{J} 02362+068$} & $\mathrm{M} 4.0 \mathrm{~V}$ & 3347 & 5.03 & -0.01 & $<3$ & $\ldots$ & CARM. \\
\hline & & 3357 & 5.03 & -0.01 & $<3$ & $\ldots$ & FEROS \\
\hline $\mathrm{J} 02442+255$ & $\mathrm{M} 3.0 \mathrm{~V}$ & 3465 & 4.96 & -0.11 & $2.5^{b}$ & $\ldots$ & CARM. \\
\hline $\mathrm{J} 02534+174$ & M3.5 V & 3424 & 5.04 & -0.23 & $<3$ & $\ldots$ & FEROS \\
\hline J02581-128 & $\mathrm{M} 2.5 \mathrm{~V}$ & 3400 & 5.07 & -0.32 & $<3$ & $\ldots$ & FEROS \\
\hline J03026-181 & $\mathrm{M} 2.5 \mathrm{~V}$ & 3494 & 4.97 & -0.19 & $<3$ & $\ldots$ & FEROS \\
\hline J03040-203 & M3.5 V & 3360 & 5.03 & -0.03 & $<3$ & $\ldots$ & FEROS \\
\hline $\mathrm{J} 03102+059$ & $\mathrm{M} 2.0 \mathrm{~V}$ & 3511 & 4.95 & -0.16 & $<3$ & $\ldots$ & FEROS \\
\hline $\mathrm{J} 03181+382$ & M1.5 V & 3533 & 5.03 & -0.45 & $2.5^{c}$ & $\ldots$ & CARM. \\
\hline J03217-066 & $\mathrm{M} 2.0 \mathrm{~V}$ & 3530 & 4.95 & -0.21 & $<3$ & $\ldots$ & FEROS \\
\hline $\mathrm{J} 03233+116$ & $\mathrm{M} 2.5 \mathrm{~V}$ & 3435 & 5.01 & -0.20 & $<3$ & Yes & FEROS \\
\hline $\mathrm{J} 03242+237$ & $\mathrm{M} 2.0 \mathrm{~V}$ & 3472 & 4.98 & -0.17 & $<3$ & $\ldots$ & CAFE \\
\hline $\mathrm{J} 03317+143$ & $\mathrm{M} 2.0 \mathrm{~V}$ & 3500 & 4.96 & -0.17 & $<3$ & $\ldots$ & FEROS \\
\hline $\mathrm{J} 03463+262$ & M0.0 V & 4025 & 4.61 & +0.28 & $<3$ & Yes & CARM. \\
\hline J03507-060 & M3.5 V & 3316 & 5.07 & -0.11 & $<3$ & $\ldots$ & FEROS \\
\hline $\mathrm{J} 03526+170$ & $\mathrm{M} 4.5 \mathrm{~V}$ & 3205 & 5.13 & -0.11 & $<3$ & $\ldots$ & FEROS \\
\hline \multirow[t]{2}{*}{$\mathrm{J} 03531+625$} & M3.0 V & 3434 & 5.00 & -0.11 & $<3$ & $\ldots$ & CAFE \\
\hline & & 3485 & 4.96 & -0.13 & $<3$ & $\ldots$ & CARM. \\
\hline J03574-011 & $\mathrm{M} 2.5 \mathrm{~V}$ & 4300 & 4.62 & -0.05 & $10.0^{e}$ & $\ldots$ & FEROS \\
\hline $\mathrm{J} 03598+260$ & $\mathrm{M} 3.0 \mathrm{~V}$ & 3414 & 5.00 & -0.06 & $<3$ & $\ldots$ & CAFE \\
\hline $\mathrm{J} 04225+105$ & M3.5 V & 3400 & 5.02 & -0.12 & $<3$ & $\ldots$ & FEROS \\
\hline J04290+219 & M0.5 V & 4169 & 4.56 & +0.41 & $1.11^{f}$ & $\ldots$ & CARM. \\
\hline \multirow[t]{3}{*}{$\mathrm{J} 04311+589$} & $\mathrm{M} 4.0 \mathrm{~V}$ & 3291 & 5.07 & -0.03 & $<3$ & $\ldots$ & CAFE \\
\hline & & 3320 & 5.07 & -0.11 & $<3$ & $\ldots$ & CARM. \\
\hline & & 3325 & 5.08 & -0.13 & $<3$ & $\ldots$ & templ. \\
\hline \multirow[t]{2}{*}{ J04376-110 } & M1.5 V & 3575 & 4.92 & -0.21 & $<3$ & $\ldots$ & CARM. \\
\hline & & 3567 & 4.90 & -0.14 & $<3$ & $\ldots$ & FEROS \\
\hline \multirow[t]{3}{*}{ J04429+189 } & $\mathrm{M} 2.0 \mathrm{~V}$ & 3522 & 4.98 & -0.26 & $<3$ & $\ldots$ & CARM. \\
\hline & & 3511 & 4.97 & -0.22 & $<3$ & $\ldots$ & FEROS \\
\hline & & 3525 & 4.98 & -0.26 & $<3$ & $\ldots$ & templ. \\
\hline \multirow[t]{2}{*}{$\mathrm{J} 04429+214$} & M3.5 V & 3395 & 5.02 & -0.09 & $<3$ & $\ldots$ & CAFE \\
\hline & & 3420 & 4.99 & -0.05 & $<3$ & $\ldots$ & FEROS \\
\hline J04538-177 & $\mathrm{M} 2.0 \mathrm{~V}$ & 3539 & 4.95 & -0.21 & $2.5^{b}$ & $\ldots$ & CARM. \\
\hline $\mathrm{J} 04544+650$ & $\mathrm{M} 4.0 \mathrm{~V}$ & 3346 & 5.10 & -0.32 & 3.4 & Yes & HRS \\
\hline \multirow[t]{2}{*}{$\mathrm{J} 04588+498$} & M0.0 V & 4111 & 4.57 & +0.41 & $<3$ & $\ldots$ & CAFE \\
\hline & & 4014 & 4.63 & +0.22 & $<3$ & Yes & CARM. \\
\hline $\mathrm{J} 05012+248$ & $\mathrm{M} 2.0 \mathrm{~V}$ & 3493 & 4.99 & -0.23 & $<3$ & $\ldots$ & CAFE \\
\hline J05019-069 & $\mathrm{M} 4.0 \mathrm{~V}$ & 3248 & 5.13 & -0.18 & $<3$ & $\ldots$ & FEROS \\
\hline
\end{tabular}


Table A.1.: Measured astrophysical parameters of investigated stars (cont.).

\begin{tabular}{|c|c|c|c|c|c|c|c|}
\hline Karmn & $\begin{array}{l}\text { Spectral } \\
\text { type }\end{array}$ & $\begin{array}{c}T_{\text {eff }}[\mathrm{K}] \\
( \pm 93)\end{array}$ & $\begin{array}{c}\log g[\mathrm{cgs}] \\
( \pm 0.29)\end{array}$ & $\begin{array}{c}{[\mathrm{Fe} / \mathrm{H}]} \\
( \pm 0.25)\end{array}$ & $\begin{array}{c}v \sin i^{a} \\
{\left[\mathrm{kms}^{-1}\right]}\end{array}$ & $\begin{array}{l}\text { Ca II } \\
\text { emis. }\end{array}$ & Instr. \\
\hline $\mathrm{J} 05034+531$ & M0.5 V & 3606 & 4.97 & -0.40 & 3.7 & $\ldots$ & CAFE \\
\hline $\mathrm{J} 05050+442$ & M5.0 V & 3298 & 5.12 & -0.27 & $<3$ & $\ldots$ & HRS \\
\hline $\mathrm{J} 05078+179$ & M3.0 V & 3429 & 5.04 & -0.27 & 3.0 & $\ldots$ & FEROS \\
\hline $\mathrm{J} 05091+154$ & $\mathrm{M} 3.0 \mathrm{~V}$ & 3437 & 5.02 & -0.23 & 4.0 & Yes & FEROS \\
\hline $\mathrm{J} 05127+196$ & $\mathrm{M} 2.0 \mathrm{~V}$ & 3547 & 4.95 & -0.23 & $2.5^{b}$ & $\ldots$ & CARM. \\
\hline \multirow[t]{2}{*}{ J05280+096 } & M3.5 V & 3375 & 5.04 & -0.14 & $<3$ & $\ldots$ & CARM. \\
\hline & & 3366 & 5.04 & -0.08 & $<3$ & $\ldots$ & FEROS \\
\hline J05298-034 & $\mathrm{M} 2.5 \mathrm{~V}$ & 3453 & 4.98 & -0.09 & $<3$ & $\ldots$ & FEROS \\
\hline $\mathrm{J} 05298+320$ & M3.0 V & 3505 & 4.88 & +0.09 & $<3$ & $\ldots$ & CAFE \\
\hline \multirow[t]{2}{*}{ J05314-036 } & M1.5 V & 3524 & 5.04 & -0.47 & $<3^{g}$ & $\ldots$ & CARM. \\
\hline & & 3521 & 5.05 & -0.48 & $<3^{g}$ & $\ldots$ & templ. \\
\hline $\mathrm{J} 05333+448$ & M3.5 V & 3346 & 5.08 & -0.20 & 4.1 & $\ldots$ & CAFE \\
\hline \multirow[t]{2}{*}{ J05365+113 } & M0.0 V & 4064 & 4.63 & +0.16 & 6.4 & Yes & CARM. \\
\hline & & 4070 & 4.63 & +0.15 & 6.4 & Yes & templ. \\
\hline $\mathrm{J} 05366+112 \mathrm{~B}$ & $\mathrm{M} 4.0 \mathrm{~V}$ & 3343 & 5.08 & -0.18 & $<3$ & $\ldots$ & FEROS \\
\hline $\mathrm{J} 05415+534$ & M1.0 V & 3622 & 4.96 & -0.39 & $2.0^{c}$ & $\ldots$ & CARM. \\
\hline $\mathrm{J} 05421+124$ & M4.0 V & 3336 & 5.05 & -0.04 & $<3$ & $\ldots$ & CARM. \\
\hline \multirow[t]{2}{*}{ J05532+242 } & M1.5 V & 3523 & 4.99 & -0.30 & $<3$ & $\ldots$ & CAFE \\
\hline & & 3560 & 4.98 & -0.34 & $<3$ & $\ldots$ & CARM. \\
\hline $\mathrm{J} 06000+027$ & $\mathrm{M} 4.0 \mathrm{~V}$ & 3330 & 5.10 & -0.27 & 4.9 & $\ldots$ & FEROS \\
\hline $\mathrm{J} 06011+595$ & M3.5 V & 3360 & 5.05 & -0.12 & $<3$ & $\ldots$ & CARM. \\
\hline $\mathrm{J} 06103+821$ & $\mathrm{M} 2.0 \mathrm{~V}$ & 3519 & 4.94 & -0.16 & $2.5^{b}$ & $\ldots$ & CARM. \\
\hline \multirow[t]{2}{*}{ J06105-218 } & M0.5 V & 3600 & 4.99 & -0.42 & $1.0^{g}$ & $\ldots$ & CARM. \\
\hline & & 3565 & 5.00 & -0.39 & $1.0^{g}$ & $\ldots$ & templ. \\
\hline J06277+093 & $\mathrm{M} 2.0 \mathrm{~V}$ & 3528 & 4.95 & -0.19 & $<3$ & $\ldots$ & FEROS \\
\hline J06307+397 & $\mathrm{M} 2.0 \mathrm{~V}$ & 3511 & 5.02 & -0.39 & $<3$ & $\ldots$ & HRS \\
\hline $\mathrm{J} 06325+641$ & $\mathrm{M} 4.0 \mathrm{~V}$ & 3443 & 5.00 & -0.16 & $<3$ & $\ldots$ & HRS \\
\hline \multirow[t]{2}{*}{$\mathrm{J} 06371+175$} & M0.0 V & 3676 & 4.98 & -0.58 & $<3$ & $\ldots$ & CARM. \\
\hline & & 3686 & 4.93 & -0.47 & $<3$ & $\ldots$ & FEROS \\
\hline $\mathrm{J} 06400+285$ & $\mathrm{M} 2.0 \mathrm{~V}$ & 3434 & 5.03 & -0.24 & $<3$ & $\ldots$ & CAFE \\
\hline \multirow[t]{2}{*}{$\mathrm{J} 06421+035$} & M3.5 V & 3431 & 5.00 & -0.10 & $<3$ & $\ldots$ & CARM. \\
\hline & & 3439 & 4.96 & -0.01 & $<3$ & $\ldots$ & FEROS \\
\hline $\mathrm{J} 06422+035$ & $\mathrm{M} 4.0 \mathrm{~V}$ & 3336 & 5.04 & -0.01 & $<3$ & $\ldots$ & FEROS \\
\hline $\mathrm{J} 06524+182$ & $\mathrm{M} 4.0 \mathrm{~V}$ & 3390 & 5.03 & -0.11 & $<3$ & $\ldots$ & FEROS \\
\hline \multirow[t]{3}{*}{$\mathrm{J} 06548+332$} & $\mathrm{M} 3.0 \mathrm{~V}$ & 3422 & 5.02 & -0.15 & $<3$ & $\ldots$ & CAFE \\
\hline & & 3437 & 5.00 & -0.14 & $<3$ & $\ldots$ & CARM. \\
\hline & & 3436 & 5.00 & -0.14 & $<3$ & $\ldots$ & templ. \\
\hline \multirow[t]{2}{*}{$\mathrm{J} 07044+682$} & $\mathrm{M} 3.0 \mathrm{~V}$ & 3394 & 5.02 & -0.09 & $<3$ & $\ldots$ & CAFE \\
\hline & & 3443 & 5.01 & -0.17 & $<3$ & $\ldots$ & CARM. \\
\hline J07081-228 & $\mathrm{M} 2.0 \mathrm{~V}$ & 3589 & 4.91 & -0.18 & $<3$ & $\ldots$ & FEROS \\
\hline
\end{tabular}


Table A.1.: Measured astrophysical parameters of investigated stars (cont.).

\begin{tabular}{|c|c|c|c|c|c|c|c|}
\hline Karmn & $\begin{array}{l}\text { Spectral } \\
\text { type }\end{array}$ & $\begin{array}{c}T_{\text {eff }}[\mathrm{K}] \\
( \pm 93)\end{array}$ & $\begin{array}{c}\log g[\mathrm{cgs}] \\
( \pm 0.29)\end{array}$ & $\begin{array}{c}{[\mathrm{Fe} / \mathrm{H}]} \\
( \pm 0.25)\end{array}$ & $\begin{array}{c}v \sin i^{a} \\
{\left[\mathrm{kms}^{-1}\right]}\end{array}$ & $\begin{array}{l}\text { Ca II } \\
\text { emis. }\end{array}$ & Instr. \\
\hline $\mathrm{J} 07119+773$ & M1.5 V & 3552 & 4.97 & -0.30 & $<3$ & $\ldots$ & CAFE \\
\hline $\mathrm{J} 07181+392$ & M0.0 V & 3607 & 4.97 & -0.40 & 3.5 & $\ldots$ & CAFE \\
\hline $\mathrm{J} 07195+328$ & M0.0 V & 4124 & 4.57 & +0.40 & $<3$ & $\ldots$ & CAFE \\
\hline \multirow[t]{3}{*}{$\mathrm{J} 07274+052$} & M3.5 V & 3387 & 5.01 & -0.02 & $<3$ & $\ldots$ & CARM. \\
\hline & & 3387 & 5.00 & +0.00 & $<3$ & $\ldots$ & FEROS \\
\hline & & 3391 & 5.01 & -0.04 & $<3$ & $\ldots$ & templ. \\
\hline J07282-187 & $\mathrm{M} 4.5 \mathrm{~V}$ & 3266 & 5.09 & -0.07 & $<3$ & $\ldots$ & FEROS \\
\hline J07287-032 & $\mathrm{M} 3.0 \mathrm{~V}$ & 3445 & 5.00 & -0.14 & $2.5^{b}$ & $\ldots$ & CARM. \\
\hline \multirow[t]{2}{*}{$\mathrm{J} 07319+362 \mathrm{~N}$} & M3.5 V & 3352 & 5.02 & +0.07 & $<3$ & $\ldots$ & CAFE \\
\hline & & 3377 & 5.05 & -0.16 & $<3$ & Yes & CARM. \\
\hline $\mathrm{J} 07349+147$ & $\mathrm{M} 3.0 \mathrm{~V}$ & 3461 & 5.01 & -0.25 & 4.8 & Yes & FEROS \\
\hline \multirow[t]{2}{*}{ J07361-031 } & $\mathrm{M} 1.0 \mathrm{~V}$ & 3628 & 5.02 & -0.55 & 3.5 & Yes & CARM. \\
\hline & & 3612 & 5.03 & -0.55 & 3.5 & Yes & FEROS \\
\hline $\mathrm{J} 07366+440$ & M3.5 V & 3372 & 5.04 & -0.10 & 3.0 & $\ldots$ & HRS \\
\hline J07386-212 & $\mathrm{M} 3.0 \mathrm{~V}$ & 3401 & 5.03 & -0.15 & $<3$ & $\ldots$ & FEROS \\
\hline $\mathrm{J} 07393+021$ & M0.0 V & 4033 & 4.62 & +0.24 & $<3$ & $\ldots$ & CARM. \\
\hline \multirow[t]{2}{*}{$\mathrm{J} 07545+085$} & $\mathrm{M} 2.5 \mathrm{~V}$ & 3483 & 5.01 & -0.28 & $<3$ & $\ldots$ & FEROS \\
\hline & & 3495 & 5.00 & -0.31 & 5.6 & $\ldots$ & HRS \\
\hline \multirow[t]{2}{*}{$\mathrm{J} 07582+413$} & M3.5 V & 3314 & 5.07 & -0.09 & $<3$ & $\ldots$ & CAFE \\
\hline & & 3350 & 5.06 & -0.12 & $<3$ & $\ldots$ & CARM. \\
\hline $\mathrm{J} 07583+496$ & M3.5 V & 3285 & 5.06 & +0.06 & $<3$ & $\ldots$ & CAFE \\
\hline $\mathrm{J} 08017+237$ & M1.5 V & 3534 & 4.98 & -0.30 & $<3$ & $\ldots$ & CAFE \\
\hline J08066+558 & $\mathrm{M} 2.0 \mathrm{~V}$ & 3478 & 5.02 & -0.30 & $<3$ & $\ldots$ & CAFE \\
\hline J08105-138 & $\mathrm{M} 2.5 \mathrm{~V}$ & 3524 & 4.95 & -0.19 & $<3$ & $\ldots$ & FEROS \\
\hline \multirow{3}{*}{$\mathrm{J} 08161+013$} & $\mathrm{M} 2.0 \mathrm{~V}$ & 3546 & 4.94 & -0.21 & $<3$ & $\ldots$ & CARM. \\
\hline & & 3543 & 4.92 & -0.13 & $<3$ & $\ldots$ & FEROS \\
\hline & & 3555 & 4.92 & -0.17 & $<3$ & $\ldots$ & templ. \\
\hline \multirow[t]{2}{*}{ J08293+039 } & $\mathrm{M} 2.5 \mathrm{~V}$ & 3525 & 4.97 & -0.23 & $<3$ & $\ldots$ & CARM. \\
\hline & & 3524 & 4.96 & -0.21 & $<3$ & $\ldots$ & FEROS \\
\hline J08313-060 & M1.5 V & 3743 & 4.72 & +0.10 & $<3$ & $\ldots$ & FEROS \\
\hline J08344-011 & M3.5 V & 3406 & 5.00 & -0.04 & $<3$ & $\ldots$ & FEROS \\
\hline $\mathrm{J} 08371+151$ & $\mathrm{M} 2.5 \mathrm{~V}$ & 3525 & 4.88 & +0.00 & $<3$ & $\ldots$ & FEROS \\
\hline $\mathrm{J} 08427+095$ & M0.0 V & 4022 & 4.62 & +0.26 & $<3$ & $\ldots$ & FEROS \\
\hline J08428+095 & $\mathrm{M} 2.5 \mathrm{~V}$ & 3476 & 4.96 & -0.11 & $<3$ & $\ldots$ & FEROS \\
\hline $\mathrm{J} 08551+015$ & M0.0 V & 4074 & 4.59 & +0.34 & $<3$ & $\ldots$ & FEROS \\
\hline $\mathrm{J} 09005+465$ & $\mathrm{M} 4.5 \mathrm{~V}$ & 3255 & 5.11 & -0.13 & $<3$ & $\ldots$ & CARM. \\
\hline $\mathrm{J} 09008+052 \mathrm{E}$ & M3.5 V & 3469 & 4.93 & +0.01 & $<3$ & $\ldots$ & FEROS \\
\hline $\mathrm{J} 09008+052 \mathrm{~W}$ & M3.0 V & 3476 & 4.92 & +0.00 & $<3$ & $\ldots$ & FEROS \\
\hline J09023+084 & $\mathrm{M} 2.5 \mathrm{~V}$ & 3516 & 4.90 & -0.04 & $<3$ & $\ldots$ & FEROS \\
\hline J09133+688 & $\mathrm{M} 2.5 \mathrm{~V}$ & 3484 & 5.00 & -0.24 & $<3$ & Yes & CAFE \\
\hline
\end{tabular}


Table A.1.: Measured astrophysical parameters of investigated stars (cont.).

\begin{tabular}{|c|c|c|c|c|c|c|c|}
\hline Karmn & $\begin{array}{l}\text { Spectral } \\
\text { type }\end{array}$ & $\begin{array}{c}T_{\text {eff }}[\mathrm{K}] \\
( \pm 93)\end{array}$ & $\begin{array}{c}\log g \text { [cgs] } \\
( \pm 0.29)\end{array}$ & $\begin{array}{c}{[\mathrm{Fe} / \mathrm{H}]} \\
( \pm 0.25)\end{array}$ & $\begin{array}{c}v \sin i^{a} \\
{\left[\mathrm{kms}^{-1}\right]}\end{array}$ & $\begin{array}{l}\text { Ca II } \\
\text { emis. }\end{array}$ & Instr. \\
\hline & & 3534 & 4.98 & -0.30 & $<3$ & Yes & CARM. \\
\hline \multirow[t]{2}{*}{ J09140+196 } & M3.0 V & 3455 & 5.03 & -0.27 & $<3$ & $\ldots$ & CARM. \\
\hline & & 3446 & 5.01 & -0.22 & $<3$ & $\ldots$ & FEROS \\
\hline $\mathrm{J} 09143+526$ & M0.0 V & 4080 & 4.62 & +0.19 & $<3$ & $\ldots$ & CAFE \\
\hline \multirow[t]{2}{*}{ J09144+526 } & M0.0 V & 3994 & 4.66 & +0.05 & $3.21^{h}$ & Yes & CARM. \\
\hline & & 4011 & 4.66 & +0.03 & $3.21^{h}$ & $\ldots$ & templ. \\
\hline \multirow[t]{2}{*}{ J09163-186 } & M1.5 V & 3570 & 4.96 & -0.30 & $<3$ & Yes & CARM. \\
\hline & & 3555 & 4.93 & -0.19 & $<3$ & $\ldots$ & FEROS \\
\hline \multirow[t]{2}{*}{ J09218-023 } & $\mathrm{M} 2.5 \mathrm{~V}$ & 3435 & 5.03 & -0.24 & $<3$ & $\ldots$ & FEROS \\
\hline & & 3509 & 5.03 & -0.40 & $<3$ & $\ldots$ & HRS \\
\hline J09288-073 & $\mathrm{M} 2.5 \mathrm{~V}$ & 3516 & 4.90 & -0.02 & $<3$ & $\ldots$ & FEROS \\
\hline \multirow[t]{2}{*}{$\mathrm{J} 09307+003$} & M3.5 V & 3407 & 5.02 & -0.12 & $<3$ & $\ldots$ & CARM. \\
\hline & & 3418 & 5.00 & -0.08 & $<3$ & $\ldots$ & FEROS \\
\hline $\mathrm{J} 09319+363$ & M0.0 V & 3942 & 4.65 & +0.18 & 3.0 & $\ldots$ & CAFE \\
\hline \multirow[t]{2}{*}{ J09411+132 } & M1.5 V & 3497 & 4.98 & -0.24 & $<3$ & $\ldots$ & CAFE \\
\hline & & 3574 & 4.93 & -0.21 & $<3$ & $\ldots$ & CARM. \\
\hline \multirow[t]{2}{*}{ J09423+559 } & M3.5 V & 3345 & 5.02 & +0.08 & $<3$ & $\ldots$ & CAFE \\
\hline & & 3399 & 5.00 & -0.03 & $<3$ & $\ldots$ & CARM. \\
\hline J09425-192 & $\mathrm{M} 2.0 \mathrm{~V}$ & 3488 & 4.95 & -0.13 & $<3$ & $\ldots$ & FEROS \\
\hline \multirow[t]{2}{*}{$\mathrm{J} 09425+700$} & $\mathrm{M} 2.0 \mathrm{~V}$ & 3491 & 4.96 & -0.14 & $10.0^{e}$ & Yes & CARM. \\
\hline & & 3461 & 4.97 & -0.11 & $10.0^{e}$ & Yes & templ. \\
\hline $\mathrm{J} 09428+700$ & $\mathrm{M} 3.0 \mathrm{~V}$ & 3436 & 5.00 & -0.14 & $2.5^{b}$ & Yes & CARM. \\
\hline \multirow{2}{*}{$\mathrm{J} 09439+269$} & $\mathrm{M} 3.5 \mathrm{~V}$ & 3408 & 5.00 & -0.04 & $<3$ & $\ldots$ & CAFE \\
\hline & & 3421 & 5.00 & -0.09 & $<3$ & $\ldots$ & CARM. \\
\hline J09447-182 & $\mathrm{M} 4.0 \mathrm{~V}$ & 3360 & 5.03 & -0.02 & $3.0^{d}$ & $\ldots$ & CARM. \\
\hline \multirow[t]{2}{*}{ J09468+760 } & M1.5 V & 3596 & 4.88 & -0.13 & $<3$ & $\ldots$ & CAFE \\
\hline & & 3699 & 4.78 & -0.04 & $<3$ & $\ldots$ & CARM. \\
\hline J09475+129 & $\mathrm{M} 4.0 \mathrm{~V}$ & 3403 & 5.00 & +0.00 & $<3$ & $\ldots$ & FEROS \\
\hline \multirow[t]{2}{*}{ J09511-123 } & M0.5 V & 3716 & 4.83 & -0.21 & $<3$ & $\ldots$ & CARM. \\
\hline & & 3752 & 4.76 & -0.05 & $<3$ & $\ldots$ & FEROS \\
\hline \multirow[t]{3}{*}{$\mathrm{J} 09561+627$} & M0.0 V & 4090 & 4.58 & +0.40 & $<3$ & $\ldots$ & CAFE \\
\hline & & 3626 & 5.04 & -0.61 & $<3$ & Yes & CARM. \\
\hline & & 3625 & 5.04 & -0.59 & $<3$ & $\ldots$ & templ. \\
\hline \multirow[t]{2}{*}{$\mathrm{J} 10023+480$} & M1.0 V & 3918 & 4.61 & +0.36 & $<3$ & $\ldots$ & CAFE \\
\hline & & 3743 & 4.75 & -0.01 & $<3$ & $\ldots$ & CARM. \\
\hline $\mathrm{J} 10087+027$ & M3.0 V & 3495 & 4.91 & +0.02 & $<3$ & $\ldots$ & FEROS \\
\hline \multirow[t]{3}{*}{ J10122-037 } & M1.5 V & 3554 & 4.97 & -0.29 & $<3$ & $\ldots$ & CARM. \\
\hline & & 3546 & 4.94 & -0.22 & $<3$ & $\ldots$ & FEROS \\
\hline & & 3565 & 4.96 & -0.29 & $<3$ & $\ldots$ & templ. \\
\hline $\mathrm{J} 10143+210$ & M0.5 V & 3604 & 5.10 & -0.72 & 6.5 & Yes & CAFE \\
\hline
\end{tabular}


Table A.1.: Measured astrophysical parameters of investigated stars (cont.).

\begin{tabular}{|c|c|c|c|c|c|c|c|}
\hline Karmn & $\begin{array}{l}\text { Spectral } \\
\text { type }\end{array}$ & $\begin{array}{c}T_{\text {eff }}[\mathrm{K}] \\
( \pm 93)\end{array}$ & $\begin{array}{c}\log g[\mathrm{cgs}] \\
( \pm 0.29)\end{array}$ & $\begin{array}{c}{[\mathrm{Fe} / \mathrm{H}]} \\
( \pm 0.25)\end{array}$ & $\begin{array}{c}v \sin i^{a} \\
{\left[\mathrm{kms}^{-1}\right]}\end{array}$ & $\begin{array}{l}\text { Ca II } \\
\text { emis. }\end{array}$ & Instr. \\
\hline $\mathrm{J} 10158+174$ & M3.5 V & 3377 & 5.03 & -0.10 & $<3$ & $\ldots$ & FEROS \\
\hline \multirow[t]{2}{*}{ J10167-119 } & $\mathrm{M} 3.0 \mathrm{~V}$ & 3531 & 4.90 & -0.05 & $<3$ & $\ldots$ & CARM. \\
\hline & & 3527 & 4.88 & +0.00 & $<3$ & $\ldots$ & FEROS \\
\hline $\mathrm{J} 10243+119$ & $\mathrm{M} 2.0 \mathrm{~V}$ & 3486 & 4.96 & -0.14 & $<3$ & $\ldots$ & FEROS \\
\hline \multirow[t]{2}{*}{ J10251-102 } & $\mathrm{M} 1.0 \mathrm{~V}$ & 3587 & 4.97 & -0.35 & $<3$ & $\ldots$ & CARM. \\
\hline & & 3575 & 4.94 & -0.23 & $<3$ & $\ldots$ & FEROS \\
\hline \multirow[t]{3}{*}{$\mathrm{J} 10289+008$} & $\mathrm{M} 2.0 \mathrm{~V}$ & 3564 & 4.92 & -0.17 & $<3$ & $\ldots$ & CARM. \\
\hline & & 3555 & 4.92 & -0.14 & $<3$ & $\ldots$ & FEROS \\
\hline & & 3574 & 4.91 & -0.16 & $<3$ & $\ldots$ & templ. \\
\hline \multirow[t]{2}{*}{ J10350-094 } & $\mathrm{M} 3.0 \mathrm{~V}$ & 3430 & 5.02 & -0.19 & $<3$ & $\ldots$ & CARM. \\
\hline & & 3426 & 5.00 & -0.12 & $<3$ & $\ldots$ & FEROS \\
\hline $\mathrm{J} 10354+694$ & M3.5 V & 3338 & 5.04 & -0.01 & $<3$ & $\ldots$ & CAFE \\
\hline \multirow[t]{2}{*}{ J10396-069 } & $\mathrm{M} 2.5 \mathrm{~V}$ & 3474 & 5.00 & -0.21 & $<3$ & $\ldots$ & CARM. \\
\hline & & 3483 & 4.98 & -0.19 & $<3$ & $\ldots$ & FEROS \\
\hline \multirow[t]{2}{*}{$\mathrm{J} 10504+331$} & $\mathrm{M} 4.0 \mathrm{~V}$ & 3334 & 5.02 & +0.11 & $<3$ & $\ldots$ & CAFE \\
\hline & & 3382 & 5.03 & -0.06 & $<3$ & $\ldots$ & CARM. \\
\hline \multirow[t]{2}{*}{$\mathrm{J} 10508+068$} & $\mathrm{M} 4.0 \mathrm{~V}$ & 3325 & 5.08 & -0.16 & $<3$ & $\ldots$ & CARM. \\
\hline & & 3335 & 5.05 & -0.07 & $<3$ & $\ldots$ & FEROS \\
\hline $\mathrm{J} 10520+139$ & M3.5 V & 3366 & 5.03 & -0.07 & $<3$ & $\ldots$ & FEROS \\
\hline $\mathrm{J} 10546-073$ & $\mathrm{M} 4.0 \mathrm{~V}$ & 3346 & 5.05 & -0.10 & $<3$ & $\ldots$ & FEROS \\
\hline \multirow[t]{2}{*}{$\mathrm{J} 11000+228$} & $\mathrm{M} 2.5 \mathrm{~V}$ & 3498 & 4.97 & -0.20 & $2.5^{b}$ & $\ldots$ & CARM. \\
\hline & & 3467 & 4.98 & -0.16 & $2.5^{b}$ & $\ldots$ & templ. \\
\hline \multirow[t]{3}{*}{$\mathrm{J} 11026+219$} & $\mathrm{M} 2.0 \mathrm{~V}$ & 3614 & 4.99 & -0.45 & 4.5 & $\ldots$ & CAFE \\
\hline & & 3621 & 5.03 & -0.56 & 4.5 & Yes & CARM. \\
\hline & & 3895 & 4.68 & +0.10 & 4.5 & Yes & templ. \\
\hline \multirow[t]{3}{*}{$\mathrm{J} 11033+359$} & M1.5 V & 3533 & 4.94 & -0.16 & $<3$ & $\ldots$ & CAFE \\
\hline & & 3596 & 4.89 & -0.14 & $<3$ & $\ldots$ & CARM. \\
\hline & & 3600 & 4.89 & -0.15 & $<3$ & $\ldots$ & templ. \\
\hline \multirow[t]{3}{*}{$\mathrm{J} 11054+435$} & $\mathrm{M} 1.0 \mathrm{~V}$ & 3583 & 4.98 & -0.37 & $<3$ & $\ldots$ & CAFE \\
\hline & & 3626 & 4.96 & -0.38 & $<3$ & $\ldots$ & CARM. \\
\hline & & 3636 & 4.94 & -0.35 & $<3$ & $\ldots$ & templ. \\
\hline $\mathrm{J} 11075+437$ & $\mathrm{M} 3.0 \mathrm{~V}$ & 3433 & 5.08 & -0.42 & 4.1 & $\ldots$ & HRS \\
\hline $\mathrm{J} 11081-052$ & $\mathrm{M} 3.0 \mathrm{~V}$ & 3472 & 4.96 & -0.08 & $<3$ & $\ldots$ & FEROS \\
\hline \multirow[t]{3}{*}{ J11110+304 } & $\mathrm{M} 2.0 \mathrm{~V}$ & 3513 & 4.99 & -0.28 & $<3$ & $\ldots$ & CAFE \\
\hline & & 4194 & 4.58 & +0.26 & $<3$ & $\ldots$ & CARM. \\
\hline & & 3832 & 4.66 & +0.22 & $<3$ & $\ldots$ & templ. \\
\hline \multirow[t]{3}{*}{ J11126+189 } & M1.5 V & 3534 & 5.00 & -0.34 & $<3$ & $\ldots$ & CAFE \\
\hline & & 3560 & 4.98 & -0.34 & $<3$ & $\ldots$ & CARM. \\
\hline & & 3554 & 4.98 & -0.33 & $<3$ & $\ldots$ & FEROS \\
\hline $\mathrm{J} 11152+194$ & M3.5 V & 3407 & 4.99 & -0.02 & $<3$ & $\ldots$ & FEROS \\
\hline
\end{tabular}


Table A.1.: Measured astrophysical parameters of investigated stars (cont.).

\begin{tabular}{|c|c|c|c|c|c|c|c|}
\hline Karmn & $\begin{array}{l}\text { Spectral } \\
\text { type }\end{array}$ & $\begin{array}{c}T_{\text {eff }}[\mathrm{K}] \\
( \pm 93)\end{array}$ & $\begin{array}{c}\log g[\mathrm{cgs}] \\
( \pm 0.29)\end{array}$ & $\begin{array}{c}{[\mathrm{Fe} / \mathrm{H}]} \\
( \pm 0.25)\end{array}$ & $\begin{array}{c}v \sin i^{a} \\
{\left[\mathrm{kms}^{-1}\right]}\end{array}$ & $\begin{array}{l}\text { Ca II } \\
\text { emis. }\end{array}$ & Instr. \\
\hline \multirow[t]{3}{*}{$\mathrm{J} 11289+101$} & $\mathrm{M} 3.5 \mathrm{~V}$ & 3336 & 5.04 & -0.02 & $<3$ & $\ldots$ & CAFE \\
\hline & & 3395 & 5.01 & -0.08 & $<3$ & $\ldots$ & CARM. \\
\hline & & 3370 & 5.02 & -0.03 & $<3$ & $\ldots$ & FEROS \\
\hline \multirow[t]{2}{*}{$\mathrm{J} 11417+427$} & $\mathrm{M} 4.0 \mathrm{~V}$ & 3364 & 5.01 & +0.07 & $<3$ & $\ldots$ & CAFE \\
\hline & & 3385 & 5.01 & -0.02 & $<3$ & $\ldots$ & CARM. \\
\hline \multirow[t]{3}{*}{$\mathrm{J} 11421+267$} & $\mathrm{M} 2.5 \mathrm{~V}$ & 3465 & 4.97 & -0.08 & $<3$ & $\ldots$ & CAFE \\
\hline & & 3500 & 4.95 & -0.13 & $<3$ & $\ldots$ & CARM. \\
\hline & & 3502 & 4.95 & -0.14 & $<3$ & $\ldots$ & templ. \\
\hline \multirow[t]{3}{*}{ J11467-140 } & M3.0 V & 3431 & 4.99 & -0.07 & $<3$ & $\ldots$ & CAFE \\
\hline & & 3470 & 4.98 & -0.14 & $<3$ & $\ldots$ & CARM. \\
\hline & & 3464 & 4.97 & -0.09 & $<3$ & $\ldots$ & FEROS \\
\hline $\mathrm{J} 11476+002$ & $\mathrm{M} 4.0 \mathrm{~V}$ & 3294 & 5.11 & -0.22 & $<3$ & Yes & FEROS \\
\hline \multirow[t]{2}{*}{$\mathrm{J} 11476+786$} & M3.5 V & 3385 & 5.02 & -0.08 & $<3$ & $\ldots$ & CARM. \\
\hline & & 3378 & 5.02 & -0.05 & $<3$ & $\ldots$ & templ. \\
\hline \multirow[t]{2}{*}{$\mathrm{J} 11477+008$} & $\mathrm{M} 4.0 \mathrm{~V}$ & 3268 & 5.11 & -0.15 & $<3$ & $\ldots$ & FEROS \\
\hline & & 3274 & 5.11 & -0.17 & $<3$ & $\ldots$ & templ. \\
\hline \multirow[t]{2}{*}{$\mathrm{J} 11509+483$} & M4.5 V & 3193 & 5.14 & -0.12 & $<3$ & $\ldots$ & CAFE \\
\hline & & 3252 & 5.12 & -0.15 & $<3$ & $\ldots$ & CARM. \\
\hline \multirow[t]{3}{*}{$\mathrm{J} 11511+352$} & M1.5 V & 3575 & 4.94 & -0.24 & $<3$ & $\ldots$ & CAFE \\
\hline & & 3612 & 4.94 & -0.30 & $<3$ & $\ldots$ & CARM. \\
\hline & & 3629 & 4.91 & -0.26 & $<3$ & $\ldots$ & templ. \\
\hline $\mathrm{J} 11521+039$ & $\mathrm{M} 4.0 \mathrm{~V}$ & 3366 & 5.05 & -0.15 & 3.1 & $\ldots$ & FEROS \\
\hline J11532-073 & $\mathrm{M} 2.5 \mathrm{~V}$ & 3503 & 4.96 & -0.16 & $<3$ & $\ldots$ & FEROS \\
\hline $\mathrm{J} 11541+098$ & M3.5 V & 3335 & 5.10 & -0.27 & $<3$ & $\ldots$ & FEROS \\
\hline J11549-021 & M3.0 V & 3434 & 5.02 & -0.19 & $<3$ & $\ldots$ & HRS \\
\hline J12006-138 & $\mathrm{M} 3.5 \mathrm{~V}$ & 3405 & 5.02 & -0.11 & $<3$ & $\ldots$ & FEROS \\
\hline J12016-122 & $\mathrm{M} 3.0 \mathrm{~V}$ & 3501 & 4.92 & -0.03 & $<3$ & $\ldots$ & FEROS \\
\hline \multirow[t]{2}{*}{$\mathrm{J} 12054+695$} & $\mathrm{M} 4.0 \mathrm{~V}$ & 3299 & 5.04 & +0.12 & $<3$ & $\ldots$ & CAFE \\
\hline & & 3359 & 5.03 & -0.02 & $<3$ & $\ldots$ & CARM. \\
\hline \multirow[t]{3}{*}{$\mathrm{J} 12100-150$} & M3.5 V & 3367 & 4.98 & +0.18 & $<3$ & $\ldots$ & CAFE \\
\hline & & 3367 & 5.02 & -0.03 & $<3$ & $\ldots$ & CARM. \\
\hline & & 3343 & 5.02 & +0.06 & $<3$ & $\ldots$ & FEROS \\
\hline J12111-199 & $\mathrm{M} 3.0 \mathrm{~V}$ & 3452 & 5.00 & -0.16 & $3.0^{d}$ & $\ldots$ & CARM. \\
\hline J12112-199 & M3.5 V & 3380 & 5.05 & -0.16 & $<3$ & $\ldots$ & FEROS \\
\hline $\mathrm{J} 12123+544 \mathrm{~N}$ & M3.0 V & 3310 & 5.06 & -0.02 & $<3$ & $\ldots$ & CAFE \\
\hline \multirow[t]{3}{*}{$\mathrm{J} 12123+544 \mathrm{~S}$} & M0.0 V & 4024 & 4.63 & +0.23 & 3.9 & $\ldots$ & CAFE \\
\hline & & 3914 & 4.69 & +0.02 & 3.9 & $\ldots$ & CARM. \\
\hline & & 3922 & 4.69 & +0.00 & 3.9 & $\ldots$ & templ. \\
\hline \multirow[t]{2}{*}{$\mathrm{J} 12230+640$} & $\mathrm{M} 3.0 \mathrm{~V}$ & 3524 & 4.84 & +0.14 & $<3$ & $\ldots$ & CAFE \\
\hline & & 3532 & 4.88 & -0.01 & $<3$ & $\ldots$ & CARM. \\
\hline
\end{tabular}


Table A.1.: Measured astrophysical parameters of investigated stars (cont.).

\begin{tabular}{|c|c|c|c|c|c|c|c|}
\hline Karmn & $\begin{array}{l}\text { Spectral } \\
\text { type }\end{array}$ & $\begin{array}{c}T_{\text {eff }}[\mathrm{K}] \\
( \pm 93)\end{array}$ & $\begin{array}{c}\log g[\mathrm{cgs}] \\
( \pm 0.29)\end{array}$ & $\begin{array}{c}{[\mathrm{Fe} / \mathrm{H}]} \\
( \pm 0.25)\end{array}$ & $\begin{array}{c}v \sin i^{a} \\
{\left[\mathrm{kms}^{-1}\right]}\end{array}$ & $\begin{array}{l}\text { Ca II } \\
\text { emis. }\end{array}$ & Instr. \\
\hline \multirow[t]{2}{*}{ J12248-182 } & $\mathrm{M} 2.0 \mathrm{~V}$ & 3500 & 4.98 & -0.22 & $<3$ & $\ldots$ & CARM. \\
\hline & & 3488 & 4.97 & -0.18 & $<3$ & $\ldots$ & FEROS \\
\hline J12277-032 & M3.5 V & 3421 & 4.99 & -0.04 & $<3$ & $\ldots$ & FEROS \\
\hline \multirow[t]{2}{*}{$\mathrm{J} 12312+086$} & M0.5 V & 3683 & 4.95 & -0.48 & $<3$ & Yes & CARM. \\
\hline & & 3974 & 4.64 & +0.19 & $<3$ & $\ldots$ & FEROS \\
\hline \multirow[t]{2}{*}{$\mathrm{J} 12350+098$} & $\mathrm{M} 2.5 \mathrm{~V}$ & 3606 & 4.83 & -0.01 & $<3$ & $\ldots$ & CARM. \\
\hline & & 3596 & 4.83 & +0.00 & $<3$ & $\ldots$ & FEROS \\
\hline \multirow[t]{2}{*}{ J12373-208 } & $\mathrm{M} 4.0 \mathrm{~V}$ & 3358 & 5.03 & -0.05 & $<3$ & $\ldots$ & CARM. \\
\hline & & 3354 & 5.03 & -0.01 & $<3$ & $\ldots$ & FEROS \\
\hline \multirow[t]{2}{*}{$\mathrm{J} 12388+116$} & $\mathrm{M} 3.0 \mathrm{~V}$ & 3444 & 4.98 & -0.08 & $<3$ & $\ldots$ & CARM. \\
\hline & & 3447 & 4.97 & -0.05 & $<3$ & $\ldots$ & FEROS \\
\hline \multirow[t]{2}{*}{$\mathrm{J} 12428+418$} & $\mathrm{M} 4.0 \mathrm{~V}$ & 3283 & 5.09 & -0.11 & 3.0 & $\ldots$ & CAFE \\
\hline & & 3344 & 5.08 & -0.21 & 3.0 & $\ldots$ & CARM. \\
\hline $\mathrm{J} 12471-035$ & $\mathrm{M} 3.0 \mathrm{~V}$ & 3399 & 5.03 & -0.15 & $<3$ & $\ldots$ & FEROS \\
\hline \multirow[t]{3}{*}{$\mathrm{J} 12479+097$} & M3.5 V & 3399 & 5.01 & -0.04 & $<3$ & $\ldots$ & CARM. \\
\hline & & 3392 & 5.00 & +0.00 & $<3$ & $\ldots$ & FEROS \\
\hline & & 3402 & 5.00 & -0.03 & $<3$ & $\ldots$ & templ. \\
\hline \multirow[t]{2}{*}{$\mathrm{J} 13000-056$} & $\mathrm{M} 3.0 \mathrm{~V}$ & 3365 & 5.03 & -0.03 & $<3$ & $\ldots$ & CAFE \\
\hline & & 3431 & 4.99 & -0.07 & $<3$ & $\ldots$ & FEROS \\
\hline $\mathrm{J} 13180+022$ & $\mathrm{M} 3.5 \mathrm{~V}$ & 3324 & 5.08 & -0.15 & 3.6 & $\ldots$ & CAFE \\
\hline $\mathrm{J} 13195+351 \mathrm{E}$ & $\mathrm{M} 3.0 \mathrm{~V}$ & 3384 & 5.06 & -0.22 & $<3$ & $\ldots$ & CAFE \\
\hline \multirow[t]{2}{*}{$\mathrm{J} 13196+333$} & M1.5 V & 3487 & 5.02 & -0.33 & $<3$ & $\ldots$ & CAFE \\
\hline & & 3525 & 5.02 & -0.39 & $<3$ & $\ldots$ & CARM. \\
\hline \multirow[t]{2}{*}{$\mathrm{J} 13209+342$} & M1.0 V & 3592 & 4.92 & -0.21 & $<3$ & $\ldots$ & CAFE \\
\hline & & 3605 & 4.92 & -0.26 & $<3$ & $\ldots$ & CARM. \\
\hline \multirow[t]{2}{*}{$\mathrm{J} 13229+244$} & $\mathrm{M} 4.0 \mathrm{~V}$ & 3263 & 5.07 & +0.09 & $<3$ & $\ldots$ & CAFE \\
\hline & & 3386 & 5.00 & +0.00 & $<3$ & $\ldots$ & CARM. \\
\hline \multirow[t]{3}{*}{$\mathrm{J} 13283-023 \mathrm{~W}$} & $\mathrm{M} 3.0 \mathrm{~V}$ & 3415 & 5.00 & -0.07 & $<3$ & $\ldots$ & CAFE \\
\hline & & 3460 & 4.98 & -0.14 & $<3$ & $\ldots$ & CARM. \\
\hline & & 3461 & 4.96 & -0.06 & $<3$ & $\ldots$ & FEROS \\
\hline $\mathrm{J} 13293+114$ & M3.5 V & 3486 & 4.90 & +0.03 & $<3$ & $\ldots$ & FEROS \\
\hline \multirow[t]{3}{*}{$\mathrm{J} 13299+102$} & M0.5 V & 3605 & 4.94 & -0.30 & $<3$ & $\ldots$ & CAFE \\
\hline & & 3644 & 4.92 & -0.32 & $<3$ & $\ldots$ & CARM. \\
\hline & & 3648 & 4.91 & -0.31 & $<3$ & $\ldots$ & templ. \\
\hline $\mathrm{J} 13343+046$ & M0.0 V & 4132 & 4.56 & +0.46 & 4.0 & $\ldots$ & FEROS \\
\hline $\mathrm{J} 13427+332$ & M3.5 V & 3356 & 5.05 & -0.13 & $4.0^{d}$ & $\ldots$ & CARM. \\
\hline $\mathrm{J} 13457+148$ & M1.5 V & 3612 & 4.86 & -0.08 & $<3$ & $\ldots$ & CAFE \\
\hline \multirow[t]{3}{*}{$\mathrm{J} 13450+176$} & $\mathrm{M} 1.0 \mathrm{~V}$ & 3816 & 4.87 & -0.50 & $2.0^{j}$ & $\ldots$ & CARM. \\
\hline & & 3696 & 4.82 & -0.16 & $<3$ & $\ldots$ & CARM. \\
\hline & & 3697 & 4.77 & +0.00 & $<3$ & $\ldots$ & FEROS \\
\hline
\end{tabular}


Table A.1.: Measured astrophysical parameters of investigated stars (cont.).

\begin{tabular}{|c|c|c|c|c|c|c|c|}
\hline Karmn & $\begin{array}{l}\text { Spectral } \\
\text { type }\end{array}$ & $\begin{array}{c}T_{\text {eff }}[\mathrm{K}] \\
( \pm 93)\end{array}$ & $\begin{array}{c}\log g \text { [cgs] } \\
( \pm 0.29)\end{array}$ & $\begin{array}{c}{[\mathrm{Fe} / \mathrm{H}]} \\
( \pm 0.25)\end{array}$ & $\begin{array}{c}v \sin i^{a} \\
{\left[\mathrm{kms}^{-1}\right]}\end{array}$ & $\begin{array}{l}\text { Ca II } \\
\text { emis. }\end{array}$ & Instr. \\
\hline & & 3689 & 4.80 & -0.08 & $<3$ & $\ldots$ & templ. \\
\hline \multirow[t]{2}{*}{ J13458-179 } & M3.5 V & 3428 & 4.98 & -0.02 & $<3$ & $\ldots$ & CARM. \\
\hline & & 3425 & 4.98 & +0.00 & $<3$ & $\ldots$ & FEROS \\
\hline \multirow[t]{2}{*}{$\mathrm{J} 13526+144$} & $\mathrm{M} 2.0 \mathrm{~V}$ & 3455 & 5.03 & -0.26 & $<3$ & $\ldots$ & CAFE \\
\hline & & 3483 & 5.01 & -0.29 & $<3$ & $\ldots$ & FEROS \\
\hline \multirow[t]{3}{*}{$\mathrm{J} 13582+125$} & $\mathrm{M} 3.0 \mathrm{~V}$ & 3340 & 5.09 & -0.24 & $<3$ & $\ldots$ & CAFE \\
\hline & & 3386 & 5.07 & -0.28 & $<3$ & $\ldots$ & CARM \\
\hline & & 3366 & 5.07 & -0.25 & $<3$ & $\ldots$ & FEROS \\
\hline \multirow[t]{3}{*}{ J14010-026 } & M1.0 V & 3772 & 4.68 & +0.19 & $<3$ & $\ldots$ & CAFE \\
\hline & & 3618 & 4.91 & -0.24 & $<3$ & $\ldots$ & CARM \\
\hline & & 3720 & 4.73 & +0.08 & $<3$ & $\ldots$ & FEROS \\
\hline $\mathrm{J} 14062+693$ & M3.0 V & 3428 & 5.01 & -0.16 & $<3$ & $\ldots$ & CAFE \\
\hline \multirow[t]{2}{*}{$\mathrm{J} 14082+805$} & M1.0 V & 3564 & 4.94 & -0.22 & $<3$ & $\ldots$ & CAFE \\
\hline & & 3615 & 4.93 & -0.27 & $<3$ & $\ldots$ & CARM. \\
\hline $\mathrm{J} 14152+450$ & M3.0 V & 3498 & 4.92 & -0.06 & $<3$ & $\ldots$ & CARM. \\
\hline $\mathrm{J} 14174+454$ & M0.0 V & 4170 & 4.56 & +0.41 & $<3$ & $\ldots$ & CAFE \\
\hline \multirow[t]{2}{*}{$\mathrm{J} 14251+518$} & $\mathrm{M} 2.5 \mathrm{~V}$ & 3473 & 4.97 & -0.13 & $<3$ & $\ldots$ & CAFE \\
\hline & & 3502 & 4.97 & -0.20 & $<3$ & $\ldots$ & CARM. \\
\hline \multirow[t]{3}{*}{$\mathrm{J} 14257+236 \mathrm{E}$} & M0.5 V & 4030 & 4.58 & +0.47 & $<3$ & $\ldots$ & CAFE \\
\hline & & 3588 & 5.04 & -0.54 & $<3$ & $\ldots$ & CARM \\
\hline & & 3583 & 5.02 & -0.47 & $<3$ & $\ldots$ & templ. \\
\hline \multirow[t]{3}{*}{$\mathrm{J} 14257+236 \mathrm{~W}$} & M0.0 V & 3584 & 5.03 & -0.52 & $<3$ & $\ldots$ & CAFE \\
\hline & & 4036 & 4.60 & +0.35 & $<3$ & $\ldots$ & CARM \\
\hline & & 4035 & 4.60 & +0.33 & $<3$ & $\ldots$ & templ. \\
\hline \multirow[t]{2}{*}{$\mathrm{J} 14283+053$} & $\mathrm{M} 3.0 \mathrm{~V}$ & 3395 & 5.03 & -0.12 & $<3$ & $\ldots$ & CAFE \\
\hline & & 3455 & 4.99 & -0.12 & $<3$ & $\ldots$ & FEROS \\
\hline \multirow[t]{2}{*}{ J14294+155 } & $\mathrm{M} 2.0 \mathrm{~V}$ & 3561 & 4.94 & -0.22 & $<3$ & $\ldots$ & CARM \\
\hline & & 3555 & 4.92 & -0.15 & $<3$ & $\ldots$ & FEROS \\
\hline \multirow[t]{2}{*}{$\mathrm{J} 14310-122$} & $\mathrm{M} 3.5 \mathrm{~V}$ & 3401 & 5.03 & -0.12 & $<3$ & $\ldots$ & CARM \\
\hline & & 3399 & 5.01 & -0.05 & $<3$ & $\ldots$ & FEROS \\
\hline $\mathrm{J} 14331+610$ & $\mathrm{M} 2.5 \mathrm{~V}$ & 3471 & 5.00 & -0.22 & 4.0 & $\ldots$ & CAFE \\
\hline \multirow[t]{3}{*}{$\mathrm{J} 14342-125$} & $\mathrm{M} 4.0 \mathrm{~V}$ & 3339 & 5.05 & -0.07 & $<3$ & $\ldots$ & CARM \\
\hline & & 3335 & 5.03 & +0.01 & $<3$ & $\ldots$ & FEROS \\
\hline & & 3348 & 5.04 & -0.05 & $<3$ & $\ldots$ & templ. \\
\hline \multirow[t]{3}{*}{$\mathrm{J} 14524+123$} & $\mathrm{M} 2.0 \mathrm{~V}$ & 3445 & 5.02 & -0.21 & $<3$ & $\ldots$ & CAFE \\
\hline & & 3481 & 5.01 & -0.27 & $<3$ & $\ldots$ & CARM. \\
\hline & & 3483 & 5.00 & -0.26 & $<3$ & $\ldots$ & FEROS \\
\hline \multirow[t]{2}{*}{$\mathrm{J} 14544+355$} & $\mathrm{M} 3.5 \mathrm{~V}$ & 3347 & 5.02 & +0.03 & $<3$ & $\ldots$ & CAFE \\
\hline & & 3413 & 5.00 & -0.03 & $<3$ & $\ldots$ & CARM \\
\hline $\mathrm{J} 15011+071$ & M3.5 V & 3453 & 4.99 & -0.14 & 6.3 & $\ldots$ & FEROS \\
\hline
\end{tabular}


Table A.1.: Measured astrophysical parameters of investigated stars (cont.).

\begin{tabular}{|c|c|c|c|c|c|c|c|}
\hline Karmn & $\begin{array}{l}\text { Spectral } \\
\text { type }\end{array}$ & $\begin{array}{c}T_{\text {eff }}[\mathrm{K}] \\
( \pm 93)\end{array}$ & $\begin{array}{c}\log g[\mathrm{cgs}] \\
( \pm 0.29)\end{array}$ & $\begin{array}{c}{[\mathrm{Fe} / \mathrm{H}]} \\
( \pm 0.25)\end{array}$ & $\begin{array}{c}v \sin i^{a} \\
{\left[\mathrm{kms}^{-1}\right]}\end{array}$ & $\begin{array}{l}\text { Ca II } \\
\text { emis. }\end{array}$ & Instr. \\
\hline \multirow[t]{3}{*}{$\mathrm{J} 15013+055$} & $\mathrm{M} 3.0 \mathrm{~V}$ & 3355 & 5.05 & -0.09 & $<3$ & $\ldots$ & CAFE \\
\hline & & 3433 & 5.01 & -0.15 & $<3$ & $\ldots$ & CARM. \\
\hline & & 3427 & 5.01 & -0.14 & $<3$ & $\ldots$ & FEROS \\
\hline $\mathrm{J} 15043+603$ & $\mathrm{M} 1.0 \mathrm{~V}$ & 3541 & 4.98 & -0.30 & $<3$ & $\ldots$ & CAFE \\
\hline $\mathrm{J} 15073+249$ & M0.0 V & 4125 & 4.60 & +0.23 & $<3$ & $\ldots$ & CAFE \\
\hline \multirow{3}{*}{$\mathrm{J} 15095+031$} & $\mathrm{M} 3.0 \mathrm{~V}$ & 3430 & 4.99 & -0.06 & $<3$ & $\ldots$ & CAFE \\
\hline & & 3459 & 4.98 & -0.13 & $<3$ & $\ldots$ & CARM. \\
\hline & & 3454 & 4.98 & -0.11 & $<3$ & $\ldots$ & FEROS \\
\hline $\mathrm{J} 15100+193$ & $\mathrm{M} 4.0 \mathrm{~V}$ & 3366 & 5.01 & +0.05 & $<3$ & $\ldots$ & FEROS \\
\hline \multirow[t]{4}{*}{ J15194-077 } & $\mathrm{M} 3.0 \mathrm{~V}$ & 3384 & 5.05 & -0.17 & $<3$ & $\ldots$ & CAFE \\
\hline & & 3436 & 5.01 & -0.16 & $<3$ & $\ldots$ & CARM. \\
\hline & & 3435 & 5.01 & -0.17 & $<3$ & $\ldots$ & FEROS \\
\hline & & 3441 & 5.01 & -0.17 & $<3$ & $\ldots$ & templ. \\
\hline $\mathrm{J} 15218+209$ & M1.5 V & 3499 & 5.02 & -0.36 & 5.1 & $\ldots$ & CAFE \\
\hline $\mathrm{J} 15357+221$ & M3.5 V & 3365 & 5.01 & +0.08 & $<3$ & $\ldots$ & CAFE \\
\hline \multirow[t]{2}{*}{ J15369-141 } & $\mathrm{M} 4.0 \mathrm{~V}$ & 3380 & 5.02 & -0.05 & $<3$ & $\ldots$ & CARM. \\
\hline & & 3371 & 5.02 & -0.02 & $<3$ & $\ldots$ & FEROS \\
\hline \multirow[t]{2}{*}{$\mathrm{J} 15412+759$} & $\mathrm{M} 3.0 \mathrm{~V}$ & 3365 & 5.08 & -0.23 & $<3$ & $\ldots$ & CAFE \\
\hline & & 3436 & 5.06 & -0.36 & $<3$ & $\ldots$ & CARM. \\
\hline J15474-108 & $\mathrm{M} 2.0 \mathrm{~V}$ & 3498 & 5.01 & -0.32 & $<3$ & $\ldots$ & CARM. \\
\hline $\mathrm{J} 15496+348$ & $\mathrm{M} 4.0 \mathrm{~V}$ & 3305 & 5.10 & -0.17 & $<3$ & $\ldots$ & CAFE \\
\hline $\mathrm{J} 15578+090$ & $\mathrm{M} 4.0 \mathrm{~V}$ & 3374 & 5.03 & -0.05 & $<3$ & $\ldots$ & FEROS \\
\hline $\mathrm{J} 15583+354$ & M3.5 V & 3370 & 5.04 & -0.11 & $<3$ & $\ldots$ & CAFE \\
\hline \multirow[t]{3}{*}{ J15598-082 } & $\mathrm{M} 1.0 \mathrm{~V}$ & 3540 & 4.97 & -0.27 & $<3$ & $\ldots$ & CAFE \\
\hline & & 3583 & 4.96 & -0.31 & $<3$ & $\ldots$ & CARM. \\
\hline & & 3589 & 4.93 & -0.24 & $<3$ & $\ldots$ & FEROS \\
\hline $\mathrm{J} 16017+301$ & $\mathrm{M} 3.0 \mathrm{~V}$ & 3415 & 5.01 & -0.10 & $<3$ & $\ldots$ & CAFE \\
\hline \multirow[t]{3}{*}{$\mathrm{J} 16028+205$} & $\mathrm{M} 4.0 \mathrm{~V}$ & 3305 & 5.06 & +0.01 & $<3$ & $\ldots$ & CAFE \\
\hline & & 3359 & 5.03 & -0.03 & $<3$ & $\ldots$ & CARM. \\
\hline & & 3330 & 5.04 & +0.01 & $<3$ & $\ldots$ & FEROS \\
\hline $\mathrm{J} 16090+529$ & M0.0 V & 4025 & 4.61 & +0.30 & $<3$ & $\ldots$ & CAFE \\
\hline \multirow[t]{3}{*}{ J16092+093 } & $\mathrm{M} 3.0 \mathrm{~V}$ & 3403 & 5.03 & -0.15 & $<3$ & $\ldots$ & CAFE \\
\hline & & 3458 & 5.00 & -0.21 & $<3$ & $\ldots$ & CARM. \\
\hline & & 3450 & 4.99 & -0.12 & $<3$ & $\ldots$ & FEROS \\
\hline \multirow[t]{2}{*}{$\mathrm{J} 16120+033$} & $\mathrm{M} 2.0 \mathrm{~V}$ & 3524 & 4.99 & -0.30 & $<3$ & $\ldots$ & CAFE \\
\hline & & 3564 & 4.98 & -0.35 & $<3$ & $\ldots$ & FEROS \\
\hline \multirow[t]{3}{*}{$\mathrm{J} 16167+672 \mathrm{~N}$} & $\mathrm{M} 3.0 \mathrm{~V}$ & 3427 & 4.99 & -0.07 & $<3$ & $\ldots$ & CAFE \\
\hline & & 3477 & 4.97 & -0.15 & $<3$ & $\ldots$ & CARM. \\
\hline & & 3482 & 4.97 & -0.14 & $<3$ & $\ldots$ & templ. \\
\hline $\mathrm{J} 16167+672 \mathrm{~S}$ & M0.0 V & 4165 & 4.56 & +0.43 & $<3$ & $\ldots$ & CAFE \\
\hline
\end{tabular}


Table A.1.: Measured astrophysical parameters of investigated stars (cont.).

\begin{tabular}{|c|c|c|c|c|c|c|c|}
\hline Karmn & $\begin{array}{l}\text { Spectral } \\
\text { type }\end{array}$ & $\begin{array}{c}T_{\text {eff }}[\mathrm{K}] \\
( \pm 93)\end{array}$ & $\begin{array}{c}\log g[\mathrm{cgs}] \\
( \pm 0.29)\end{array}$ & $\begin{array}{c}{[\mathrm{Fe} / \mathrm{H}]} \\
( \pm 0.25)\end{array}$ & $\begin{array}{c}v \sin i^{a} \\
{\left[\mathrm{kms}^{-1}\right]}\end{array}$ & $\begin{array}{l}\text { Ca II } \\
\text { emis. }\end{array}$ & Instr. \\
\hline & & 4084 & 4.60 & +0.31 & $<3$ & $\ldots$ & CARM. \\
\hline & & 4095 & 4.60 & +0.28 & $<3$ & $\ldots$ & templ. \\
\hline \multirow[t]{3}{*}{$\mathrm{J} 16254+543$} & M1.5 V & 3481 & 5.02 & -0.32 & $<3$ & $\ldots$ & CAFE \\
\hline & & 3528 & 4.99 & -0.33 & $<3$ & $\ldots$ & CARM. \\
\hline & & 3526 & 5.00 & -0.35 & $<3$ & $\ldots$ & templ. \\
\hline \multirow[t]{3}{*}{ J16303-126 } & M3.5 V & 3382 & 5.04 & -0.12 & $<3$ & $\ldots$ & CARM. \\
\hline & & 3362 & 5.04 & -0.06 & $<3$ & $\ldots$ & FEROS \\
\hline & & 3388 & 5.03 & -0.11 & $<3$ & $\ldots$ & templ. \\
\hline \multirow[t]{3}{*}{$\mathrm{J} 16327+126$} & $\mathrm{M} 3.0 \mathrm{~V}$ & 3463 & 4.94 & +0.02 & $<3$ & $\ldots$ & CAFE \\
\hline & & 3499 & 4.92 & -0.05 & $<3$ & $\ldots$ & CARM \\
\hline & & 3506 & 4.90 & -0.01 & $<3$ & $\ldots$ & FEROS \\
\hline \multirow[t]{3}{*}{$\mathrm{J} 16462+164$} & $\mathrm{M} 2.5 \mathrm{~V}$ & 3430 & 5.01 & -0.14 & $<3$ & $\ldots$ & CAFE \\
\hline & & 3486 & 4.97 & -0.17 & $<3$ & $\ldots$ & CARM. \\
\hline & & 3481 & 4.95 & -0.08 & $<3$ & $\ldots$ & FEROS \\
\hline J16487-157 & M1.5 V & 3563 & 4.97 & -0.31 & $<3$ & $\ldots$ & FEROS \\
\hline $\mathrm{J} 16554-083 \mathrm{~N}$ & $\mathrm{M} 3.5 \mathrm{~V}$ & 3355 & 5.06 & -0.17 & $2.7^{k}$ & $\ldots$ & CARM \\
\hline \multirow[t]{3}{*}{$\mathrm{J} 16581+257$} & M1.0 V & 3563 & 4.97 & -0.31 & $<3$ & $\ldots$ & CAFE \\
\hline & & 3612 & 4.95 & -0.34 & $<3$ & $\ldots$ & CARM. \\
\hline & & 3614 & 4.95 & -0.34 & $<3$ & $\ldots$ & templ. \\
\hline \multirow{3}{*}{$\mathrm{J} 17052-050$} & M1.5 V & 3589 & 4.87 & -0.07 & $<3$ & $\ldots$ & CAFE \\
\hline & & 3677 & 4.78 & -0.01 & $<3$ & $\ldots$ & CARM. \\
\hline & & 3678 & 4.79 & -0.02 & $<3$ & $\ldots$ & templ. \\
\hline \multirow[t]{2}{*}{$\mathrm{J} 17071+215$} & $\mathrm{M} 3.0 \mathrm{~V}$ & 3425 & 5.01 & -0.15 & $<3$ & $\ldots$ & CAFE \\
\hline & & 3450 & 5.01 & -0.22 & $<3$ & $\ldots$ & CARM. \\
\hline \multirow[t]{2}{*}{$\mathrm{J} 17115+384$} & M3.5 V & 3376 & 5.02 & -0.03 & $<3$ & $\ldots$ & CAFE \\
\hline & & 3415 & 5.01 & -0.12 & $<3$ & $\ldots$ & CARM. \\
\hline $\mathrm{J} 17158+190$ & M0.5 V & 3591 & 5.03 & -0.53 & 5.5 & $\ldots$ & CAFE \\
\hline \multirow[t]{2}{*}{$\mathrm{J} 17160+110$} & M1.0 V & 3903 & 4.62 & +0.34 & $<3$ & $\ldots$ & CAFE \\
\hline & & 3571 & 4.96 & -0.31 & $<3$ & $\ldots$ & FEROS \\
\hline \multirow[t]{2}{*}{$\mathrm{J} 17166+080$} & $\mathrm{M} 2.0 \mathrm{~V}$ & 3472 & 4.99 & -0.19 & $<3$ & $\ldots$ & CAFE \\
\hline & & 3518 & 4.96 & -0.21 & $<3$ & $\ldots$ & CARM \\
\hline \multirow[t]{2}{*}{$\mathrm{J} 17198+417$} & $\mathrm{M} 2.5 \mathrm{~V}$ & 3435 & 5.00 & -0.13 & $<3$ & $\ldots$ & CAFE \\
\hline & & 3486 & 4.98 & -0.20 & $<3$ & $\ldots$ & CARM. \\
\hline $\mathrm{J} 17199+265$ & M3.5 V & 3335 & 5.07 & -0.12 & $<3$ & Yes & CAFE \\
\hline \multirow[t]{2}{*}{$\mathrm{J} 17303+055$} & M0.0 V & 3595 & 5.02 & -0.50 & 3.3 & $\ldots$ & CAFE \\
\hline & & 3653 & 4.96 & -0.44 & 3.3 & $\ldots$ & CARM. \\
\hline \multirow[t]{2}{*}{$J 17355+616$} & M0.5 V & 4014 & 4.60 & +0.36 & 3.2 & $\ldots$ & CAFE \\
\hline & & 3625 & 4.98 & -0.44 & 3.2 & Yes & CARM. \\
\hline \multirow{2}{*}{$\mathrm{J} 17378+185$} & M1.0 V & 3585 & 4.96 & -0.31 & 3.0 & $\ldots$ & CAFE \\
\hline & & 3631 & 4.92 & -0.30 & 3.0 & $\ldots$ & CARM. \\
\hline
\end{tabular}


Table A.1.: Measured astrophysical parameters of investigated stars (cont.).

\begin{tabular}{|c|c|c|c|c|c|c|c|}
\hline Karmn & $\begin{array}{l}\text { Spectral } \\
\text { type }\end{array}$ & $\begin{array}{c}T_{\text {eff }}[\mathrm{K}] \\
( \pm 93)\end{array}$ & $\begin{array}{c}\log g[\mathrm{cgs}] \\
( \pm 0.29)\end{array}$ & $\begin{array}{c}{[\mathrm{Fe} / \mathrm{H}]} \\
( \pm 0.25)\end{array}$ & $\begin{array}{c}v \sin i^{a} \\
{\left[\mathrm{kms}^{-1}\right]}\end{array}$ & $\begin{array}{l}\text { Ca II } \\
\text { emis. }\end{array}$ & Instr. \\
\hline \multirow[t]{2}{*}{$\mathrm{J} 17395+277 \mathrm{~N}$} & $\mathrm{M} 3.0 \mathrm{~V}$ & 3426 & 5.00 & -0.12 & 3.9 & $\ldots$ & CAFE \\
\hline & & 3443 & 4.99 & -0.12 & 3.9 & $\ldots$ & FEROS \\
\hline $\mathrm{J} 17439+433$ & $\mathrm{M} 2.5 \mathrm{~V}$ & 3438 & 5.01 & -0.17 & $<3$ & $\ldots$ & CAFE \\
\hline $\mathrm{J} 17530+169$ & $\mathrm{M} 3.0 \mathrm{~V}$ & 3396 & 5.04 & -0.19 & $<3$ & $\ldots$ & FEROS \\
\hline \multirow[t]{2}{*}{$\mathrm{J} 17542+073$} & $\mathrm{M} 4.0 \mathrm{~V}$ & 3377 & 5.01 & -0.01 & $<3$ & $\ldots$ & CARM. \\
\hline & & 3370 & 5.02 & +0.00 & $<3$ & $\ldots$ & FEROS \\
\hline \multirow[t]{4}{*}{$\mathrm{J} 17578+046$} & M3.5 V & 3230 & 5.14 & -0.24 & $<3$ & $\ldots$ & CAFE \\
\hline & & 3300 & 5.11 & -0.25 & $<3$ & $\ldots$ & CARM. \\
\hline & & 3289 & 5.11 & -0.21 & $<3$ & $\ldots$ & FEROS \\
\hline & & 3296 & 5.10 & -0.20 & $<3$ & $\ldots$ & templ. \\
\hline \multirow{2}{*}{$\mathrm{J} 17578+465$} & $\mathrm{M} 2.5 \mathrm{~V}$ & 3391 & 5.03 & -0.12 & $<3$ & $\ldots$ & CAFE \\
\hline & & 3449 & 5.00 & -0.17 & $<3$ & $\ldots$ & CARM. \\
\hline $\mathrm{J} 18051-030$ & $\mathrm{M} 1.0 \mathrm{~V}$ & 3645 & 4.90 & -0.27 & $1.6^{c}$ & $\ldots$ & CARM. \\
\hline $\mathrm{J} 18163+015$ & M3.0 V & 3436 & 5.01 & -0.17 & $<3$ & $\ldots$ & FEROS \\
\hline $\mathrm{J} 18165+455$ & M0.5 V & 3581 & 5.03 & -0.52 & 3.6 & $\ldots$ & CAFE \\
\hline \multirow[t]{2}{*}{$\mathrm{J} 18180+387 \mathrm{E}$} & $\mathrm{M} 3.0 \mathrm{~V}$ & 3395 & 5.03 & -0.13 & $<3$ & $\ldots$ & CAFE \\
\hline & & 3454 & 4.99 & -0.15 & $<3$ & $\ldots$ & CARM. \\
\hline \multirow[t]{2}{*}{$\mathrm{J} 18221+063$} & $\mathrm{M} 4.0 \mathrm{~V}$ & 3435 & 4.98 & -0.06 & $<3$ & $\ldots$ & CARM. \\
\hline & & 3422 & 5.00 & -0.07 & $<3$ & $\ldots$ & FEROS \\
\hline $\mathrm{J} 18224+620$ & $\mathrm{M} 4.0 \mathrm{~V}$ & 3236 & 5.12 & -0.13 & $2.3^{k}$ & $\ldots$ & CARM. \\
\hline $\mathrm{J} 18240+016$ & $\mathrm{M} 2.0 \mathrm{~V}$ & 3499 & 4.96 & -0.18 & $<3$ & $\ldots$ & FEROS \\
\hline $\mathrm{J} 18312+068$ & $\mathrm{M} 1.0 \mathrm{~V}$ & 3648 & 4.87 & -0.20 & $<3$ & $\ldots$ & FEROS \\
\hline \multirow[t]{2}{*}{$\mathrm{J} 18319+406$} & M3.5 V & 3370 & 5.05 & -0.14 & $<3$ & $\ldots$ & CAFE \\
\hline & & 3418 & 5.03 & -0.21 & $<3$ & $\ldots$ & CARM. \\
\hline $\mathrm{J} 18346+401$ & M3.5 V & 3375 & 5.04 & -0.10 & $2.5^{b}$ & $\ldots$ & CARM. \\
\hline $\mathrm{J} 18353+457$ & M0.5 V & 3910 & 4.67 & +0.09 & $1.0^{l}$ & $\ldots$ & CARM. \\
\hline $\mathrm{J} 18363+136$ & $\mathrm{M} 4.0 \mathrm{~V}$ & 3332 & 5.08 & -0.17 & $<3$ & $\ldots$ & FEROS \\
\hline J18409-133 & $\mathrm{M} 1.0 \mathrm{~V}$ & 3581 & 4.97 & -0.35 & $3.0^{c}$ & $\ldots$ & CARM. \\
\hline $\mathrm{J} 18419+318$ & $\mathrm{M} 3.0 \mathrm{~V}$ & 3446 & 5.01 & -0.22 & $2.5^{b}$ & $\ldots$ & CARM. \\
\hline $\mathrm{J} 18427+139$ & $\mathrm{M} 4.0 \mathrm{~V}$ & 3300 & 5.11 & -0.25 & $<3$ & Yes & FEROS \\
\hline $\mathrm{J} 18507+479$ & M3.5 V & 3400 & 4.99 & +0.01 & $<3$ & $\ldots$ & CAFE \\
\hline $\mathrm{J} 18518+165$ & M0.0 V & 3673 & 4.92 & -0.39 & $<3$ & $\ldots$ & FEROS \\
\hline \multirow[t]{2}{*}{$\mathrm{J} 18580+059$} & M0.5 V & 3619 & 4.99 & -0.46 & $<3$ & $\ldots$ & CARM. \\
\hline & M0.5 V & 3934 & 4.62 & +0.30 & $<3$ & $\ldots$ & FEROS \\
\hline \multirow[t]{2}{*}{$\mathrm{J} 19032+034$} & $\mathrm{M} 3.0 \mathrm{~V}$ & 3450 & 4.98 & -0.10 & $<3$ & $\ldots$ & CAFE \\
\hline & & 3478 & 4.97 & -0.14 & $<3$ & $\ldots$ & FEROS \\
\hline $\mathrm{J} 19070+208$ & $\mathrm{M} 2.0 \mathrm{~V}$ & 3532 & 4.98 & -0.28 & $<3$ & $\ldots$ & CARM. \\
\hline \multirow[t]{2}{*}{$\mathrm{J} 19072+208$} & $\mathrm{M} 2.0 \mathrm{~V}$ & 3480 & 5.01 & -0.28 & $<3$ & $\ldots$ & CAFE \\
\hline & & 3528 & 4.98 & -0.28 & $<3$ & $\ldots$ & CARM. \\
\hline J19084+322 & $\mathrm{M} 3.0 \mathrm{~V}$ & 3377 & 5.04 & -0.14 & $<3$ & $\ldots$ & CAFE \\
\hline
\end{tabular}


Table A.1.: Measured astrophysical parameters of investigated stars (cont.).

\begin{tabular}{|c|c|c|c|c|c|c|c|}
\hline Karmn & $\begin{array}{l}\text { Spectral } \\
\text { type }\end{array}$ & $\begin{array}{c}T_{\mathrm{eff}}[\mathrm{K}] \\
( \pm 93)\end{array}$ & $\begin{array}{c}\log g \text { [cgs] } \\
( \pm 0.29)\end{array}$ & $\begin{array}{c}{[\mathrm{Fe} / \mathrm{H}]} \\
( \pm 0.25)\end{array}$ & $\begin{array}{c}v \sin i^{a} \\
{\left[\mathrm{kms}^{-1}\right]}\end{array}$ & $\begin{array}{l}\text { Ca II } \\
\text { emis. }\end{array}$ & Instr. \\
\hline & & 3435 & 5.01 & -0.14 & $<3$ & $\ldots$ & CARM. \\
\hline $\mathrm{J} 19098+176$ & M4.5 V & 3267 & 5.08 & -0.02 & $<3$ & $\ldots$ & FEROS \\
\hline \multirow[t]{2}{*}{$\mathrm{J} 19169+051 \mathrm{~N}$} & $\mathrm{M} 2.5 \mathrm{~V}$ & 3466 & 4.96 & -0.09 & $<3$ & $\ldots$ & CAFE \\
\hline & & 3514 & 4.96 & -0.18 & $<3$ & $\ldots$ & CARM. \\
\hline J19216+208 & $\mathrm{M} 4.5 \mathrm{~V}$ & 3285 & 5.09 & -0.10 & 3.5 & $\ldots$ & CARM. \\
\hline \multirow{2}{*}{$\mathrm{J} 19220+070$} & M3.0 V & 3324 & 5.09 & -0.22 & $<3$ & $\ldots$ & CAFE \\
\hline & & 3379 & 5.06 & -0.22 & $<3$ & $\ldots$ & FEROS \\
\hline \multirow[t]{2}{*}{$\mathrm{J} 19251+283$} & M3.0 V & 3393 & 5.04 & -0.16 & $<3$ & $\ldots$ & CARM. \\
\hline & & 3400 & 5.03 & -0.14 & $<3$ & $\ldots$ & FEROS \\
\hline $\mathrm{J} 19346+045$ & M0.0 V & 4059 & 4.66 & -0.03 & 3.3 & $\ldots$ & CARM. \\
\hline J19354+377 & M3.5 V & 3344 & 5.07 & -0.14 & 4.4 & Yes & CAFE \\
\hline $\mathrm{J} 19463+320$ & M0.5 V & 3963 & 4.61 & +0.36 & $<3$ & $\ldots$ & CAFE \\
\hline J19464+320 & $\mathrm{M} 2.5 \mathrm{~V}$ & 3487 & 4.97 & -0.16 & 3.0 & $\ldots$ & CAFE \\
\hline $\mathrm{J} 19582+650$ & M3.5 V & 3334 & 5.08 & -0.16 & $<3$ & $\ldots$ & CAFE \\
\hline $\mathrm{J} 20011+002$ & $\mathrm{M} 2.0 \mathrm{~V}$ & 3527 & 4.97 & -0.26 & $<3$ & $\ldots$ & FEROS \\
\hline J20039-081 & $\mathrm{M} 4.0 \mathrm{~V}$ & 3347 & 5.04 & -0.06 & $<3$ & $\ldots$ & FEROS \\
\hline \multirow[t]{2}{*}{$\mathrm{J} 20187+158$} & $\mathrm{M} 2.5 \mathrm{~V}$ & 3454 & 4.98 & -0.11 & $<3$ & $\ldots$ & CAFE \\
\hline & & 3506 & 4.95 & -0.16 & $<3$ & $\ldots$ & FEROS \\
\hline \multirow[t]{2}{*}{$\mathrm{J} 20305+654$} & $\mathrm{M} 2.5 \mathrm{~V}$ & 3413 & 5.01 & -0.10 & $<3$ & $\ldots$ & CAFE \\
\hline & & 3478 & 4.98 & -0.19 & $<3$ & $\ldots$ & CARM. \\
\hline \multirow[t]{2}{*}{$\mathrm{J} 20336+617$} & $\mathrm{M} 4.0 \mathrm{~V}$ & 3350 & 5.00 & +0.12 & $<3$ & $\ldots$ & CAFE \\
\hline & & 3386 & 5.01 & -0.02 & $<3$ & $\ldots$ & CARM. \\
\hline J20407+199 & $\mathrm{M} 2.5 \mathrm{~V}$ & 3477 & 4.97 & -0.15 & $<3$ & $\ldots$ & FEROS \\
\hline J20429-189 & M1.5 V & 4015 & 4.52 & +0.77 & $<3$ & $\ldots$ & CAFE \\
\hline $\mathrm{J} 20450+444$ & M1.5 V & 3482 & 5.01 & -0.27 & $<3$ & $\ldots$ & CAFE \\
\hline J20488+197 & $\mathrm{M} 4.0 \mathrm{~V}$ & 3386 & 5.03 & -0.09 & $<3$ & $\ldots$ & FEROS \\
\hline \multirow[t]{3}{*}{ J20525-169 } & $\mathrm{M} 4.0 \mathrm{~V}$ & 3268 & 5.09 & -0.05 & $<3$ & $\ldots$ & CAFE \\
\hline & & 3328 & 5.07 & -0.12 & $<3$ & $\ldots$ & CARM. \\
\hline & & 3318 & 5.07 & -0.09 & $<3$ & $\ldots$ & FEROS \\
\hline \multirow[t]{2}{*}{$\mathrm{J} 20533+621$} & M0.5 V & 3574 & 4.99 & -0.38 & $<3$ & $\ldots$ & CAFE \\
\hline & & 3643 & 4.92 & -0.30 & $<3$ & $\ldots$ & CARM. \\
\hline J20556-140N & $\mathrm{M} 4.0 \mathrm{~V}$ & 3289 & 5.07 & -0.02 & $<3$ & $\ldots$ & CAFE \\
\hline \multirow[t]{3}{*}{ J20567-104 } & $\mathrm{M} 2.5 \mathrm{~V}$ & 3422 & 5.01 & -0.11 & $<3$ & $\ldots$ & CAFE \\
\hline & & 3475 & 4.99 & -0.17 & $<3$ & $\ldots$ & CARM. \\
\hline & & 3474 & 4.96 & -0.10 & $<3$ & $\ldots$ & FEROS \\
\hline \multirow[t]{3}{*}{ J21019-063 } & $\mathrm{M} 2.5 \mathrm{~V}$ & 3450 & 5.01 & -0.22 & $<3$ & $\ldots$ & CAFE \\
\hline & & 3450 & 5.03 & -0.29 & $<3$ & $\ldots$ & CARM. \\
\hline & & 3475 & 4.97 & -0.14 & $<3$ & $\ldots$ & FEROS \\
\hline $\mathrm{J} 21055+061$ & M3.0 V & 3415 & 5.02 & -0.14 & $<3$ & $\ldots$ & FEROS \\
\hline $\mathrm{J} 21057+502$ & M3.5 V & 3485 & 4.96 & -0.11 & $<3$ & $\ldots$ & HRS \\
\hline
\end{tabular}


Table A.1.: Measured astrophysical parameters of investigated stars (cont.).

\begin{tabular}{|c|c|c|c|c|c|c|c|}
\hline Karmn & $\begin{array}{l}\text { Spectral } \\
\text { type }\end{array}$ & $\begin{array}{c}T_{\text {eff }}[\mathrm{K}] \\
( \pm 93)\end{array}$ & $\begin{array}{c}\log g[\mathrm{cgs}] \\
( \pm 0.29)\end{array}$ & $\begin{array}{c}{[\mathrm{Fe} / \mathrm{H}]} \\
( \pm 0.25)\end{array}$ & $\begin{array}{c}v \sin i^{a} \\
{\left[\mathrm{kms}^{-1}\right]}\end{array}$ & $\begin{array}{l}\text { Ca II } \\
\text { emis. }\end{array}$ & Instr. \\
\hline $\mathrm{J} 21127-073$ & $\mathrm{M} 3.5 \mathrm{~V}$ & 3449 & 5.02 & -0.24 & $<3$ & $\ldots$ & HRS \\
\hline \multirow[t]{2}{*}{$\mathrm{J} 21152+257$} & $\mathrm{M} 3.0 \mathrm{~V}$ & 3473 & 4.98 & -0.15 & $<3$ & $\ldots$ & FEROS \\
\hline & & 3455 & 5.02 & -0.23 & $<3$ & $\ldots$ & CARM. \\
\hline \multirow{3}{*}{$\mathrm{J} 21164+025$} & $\mathrm{M} 3.0 \mathrm{~V}$ & 3404 & 5.03 & -0.14 & $<3$ & $\ldots$ & CAFE \\
\hline & & 3458 & 5.00 & -0.18 & $<3$ & $\ldots$ & CARM. \\
\hline & & 3453 & 4.99 & -0.13 & $<3$ & $\ldots$ & FEROS \\
\hline \multirow[t]{3}{*}{$\mathrm{J} 21221+229$} & $\mathrm{M} 1.0 \mathrm{~V}$ & 3581 & 4.96 & -0.33 & 3.7 & $\ldots$ & CAFE \\
\hline & & 3628 & 4.95 & -0.38 & 3.7 & $\ldots$ & CARM. \\
\hline & & 3642 & 4.88 & -0.20 & 3.7 & $\ldots$ & FEROS \\
\hline $\mathrm{J} 21323+245$ & M3.5 V & 3335 & 5.09 & -0.23 & $<3$ & $\ldots$ & FEROS \\
\hline \multirow[t]{2}{*}{$\mathrm{J} 21348+515$} & $\mathrm{M} 3.0 \mathrm{~V}$ & 3769 & 4.57 & +0.53 & $<3$ & $\ldots$ & CAFE \\
\hline & & 3500 & 4.93 & -0.06 & $<3$ & $\ldots$ & CARM. \\
\hline $\mathrm{J} 21442+066$ & $\mathrm{M} 3.0 \mathrm{~V}$ & 3433 & 5.01 & -0.17 & $<3$ & $\ldots$ & FEROS \\
\hline \multirow[t]{2}{*}{$\mathrm{J} 21463+382$} & $\mathrm{M} 4.0 \mathrm{~V}$ & 3257 & 5.11 & -0.11 & $<3$ & $\ldots$ & CAFE \\
\hline & & 3326 & 5.07 & -0.11 & $<3$ & $\ldots$ & CARM. \\
\hline \multirow{2}{*}{$\mathrm{J} 21466+668$} & $\mathrm{M} 4.0 \mathrm{~V}$ & 3374 & 5.04 & -0.08 & $<3$ & $\ldots$ & CARM. \\
\hline & & 3323 & 5.05 & +0.02 & $<3$ & $\ldots$ & HRS \\
\hline $\mathrm{J} 21472-047$ & $\mathrm{M} 4.5 \mathrm{~V}$ & 3279 & 5.12 & -0.25 & $<3$ & $\ldots$ & HRS \\
\hline \multirow[t]{2}{*}{$\mathrm{J} 21574+081$} & M1.5 V & 3916 & 4.58 & +0.47 & $<3$ & $\ldots$ & CAFE \\
\hline & & 3888 & 4.59 & +0.46 & $<3$ & $\ldots$ & FEROS \\
\hline $\mathrm{J} 21584+755$ & M0.5 V & 4113 & 4.52 & 0.70 & 3.8 & $\ldots$ & CAFE \\
\hline J22020-194 & M3.5 V & 3405 & 5.01 & -0.08 & $<3$ & $\ldots$ & FEROS \\
\hline \multirow[t]{3}{*}{$\mathrm{J} 22021+014$} & M0.5 V & 4033 & 4.61 & +0.32 & $<3$ & $\ldots$ & CAFE \\
\hline & & 3633 & 4.98 & -0.45 & $<3$ & $\ldots$ & CARM. \\
\hline & & 3612 & 5.01 & -0.49 & $<3$ & $\ldots$ & FEROS \\
\hline \multirow[t]{2}{*}{$\mathrm{J} 22057+656$} & M3.5 V & 3494 & 5.03 & -0.37 & 3.9 & $\ldots$ & CAFE \\
\hline & & 3557 & 5.00 & -0.40 & 3.9 & $\ldots$ & CARM. \\
\hline J22058-119 & M0.0 V & 3625 & 5.03 & -0.58 & 3.3 & $\ldots$ & CAFE \\
\hline \multirow[t]{2}{*}{ J22096-046 } & M3.5 V & 3401 & 5.03 & -0.14 & $<3$ & $\ldots$ & CAFE \\
\hline & & 3434 & 5.01 & -0.14 & $<3$ & $\ldots$ & CARM. \\
\hline $\mathrm{J} 22114+409$ & M5.5 V & 3221 & 5.14 & -0.18 & 3.3 & $\ldots$ & HRS \\
\hline \multirow[t]{2}{*}{$\mathrm{J} 22115+184$} & $\mathrm{M} 2.0 \mathrm{~V}$ & 3449 & 5.04 & -0.30 & $<3$ & $\ldots$ & CAFE \\
\hline & & 3472 & 5.04 & -0.36 & $<3$ & $\ldots$ & CARM. \\
\hline $\mathrm{J} 22125+085$ & $\mathrm{M} 3.0 \mathrm{~V}$ & 3466 & 4.97 & -0.12 & $<3$ & $\ldots$ & FEROS \\
\hline J22137-176 & $\mathrm{M} 4.5 \mathrm{~V}$ & 3236 & 5.10 & -0.02 & $<3$ & $\ldots$ & FEROS \\
\hline J22231-176 & $\mathrm{M} 4.5 \mathrm{~V}$ & 3213 & 5.14 & -0.17 & $<3$ & Yes & FEROS \\
\hline $\mathrm{J} 22426+176$ & $\mathrm{M} 2.5 \mathrm{~V}$ & 3455 & 5.00 & -0.17 & $<3$ & $\ldots$ & FEROS \\
\hline J22532-142 & $\mathrm{M} 4.0 \mathrm{~V}$ & 3371 & 5.04 & -0.09 & $2.5^{b}$ & $\ldots$ & CARM. \\
\hline $\mathrm{J} 22559+178$ & $\mathrm{M} 1.0 \mathrm{~V}$ & 3594 & 4.96 & -0.33 & $<3$ & $\ldots$ & FEROS \\
\hline $\mathrm{J} 22565+165$ & M1.5 V & 3540 & 5.00 & -0.38 & $2.5^{b}$ & $\ldots$ & CARM. \\
\hline
\end{tabular}


Table A.1.: Measured astrophysical parameters of investigated stars (cont.).

\begin{tabular}{|c|c|c|c|c|c|c|c|}
\hline Karmn & $\begin{array}{l}\text { Spectral } \\
\text { type }\end{array}$ & $\begin{array}{c}T_{\text {eff }}[\mathrm{K}] \\
( \pm 93)\end{array}$ & $\begin{array}{c}\log g[\mathrm{cgs}] \\
( \pm 0.29)\end{array}$ & $\begin{array}{c}{[\mathrm{Fe} / \mathrm{H}]} \\
( \pm 0.25)\end{array}$ & $\begin{array}{c}v \sin i^{a} \\
{\left[\mathrm{kms}^{-1}\right]}\end{array}$ & $\begin{array}{l}\text { Ca II } \\
\text { emis. }\end{array}$ & Instr. \\
\hline $\mathrm{J} 23175+063$ & $\mathrm{M} 3.0 \mathrm{~V}$ & 3459 & 4.97 & -0.10 & $<3$ & $\ldots$ & FEROS \\
\hline $\mathrm{J} 23216+172$ & $\mathrm{M} 4.0 \mathrm{~V}$ & 3379 & 5.01 & +0.00 & $<3$ & $\ldots$ & FEROS \\
\hline $\mathrm{J} 23234+155$ & $\mathrm{M} 2.0 \mathrm{~V}$ & 3587 & 4.90 & -0.15 & $<3$ & $\ldots$ & FEROS \\
\hline $\mathrm{J} 23245+578$ & M1.0 V & 3541 & 5.02 & -0.45 & $0.5^{c}$ & $\cdots$ & CARM. \\
\hline $\mathrm{J} 23340+001$ & $\mathrm{M} 2.5 \mathrm{~V}$ & 3561 & 4.86 & +0.00 & $<3$ & $\ldots$ & FEROS \\
\hline $\mathrm{J} 23381-162$ & $\mathrm{M} 2.0 \mathrm{~V}$ & 3533 & 4.94 & -0.16 & $<3$ & $\ldots$ & FEROS \\
\hline \multirow[t]{3}{*}{$\mathrm{J} 23492+024$} & $\mathrm{M} 1.0 \mathrm{~V}$ & 3621 & 4.88 & -0.17 & $<3$ & $\ldots$ & CAFE \\
\hline & & 3655 & 4.86 & -0.19 & $<3$ & $\ldots$ & CARM. \\
\hline & & 3687 & 4.79 & -0.05 & $<3$ & $\ldots$ & FEROS \\
\hline $\mathrm{J} 23505-095$ & $\mathrm{M} 4.0 \mathrm{~V}$ & 3323 & 5.06 & -0.03 & $<3$ & $\ldots$ & FEROS \\
\hline $\mathrm{J} 23556-061$ & $\mathrm{M} 2.5 \mathrm{~V}$ & 3502 & 4.96 & -0.16 & $<3$ & $\ldots$ & FEROS \\
\hline $\mathrm{J} 23585+076$ & $\mathrm{M} 3.0 \mathrm{~V}$ & 3481 & 4.95 & -0.07 & $<3$ & $\ldots$ & FEROS \\
\hline
\end{tabular}

${ }^{a}$ Rotational velocities $(v \sin i)$ from Jeffers et al. (submitted), if no other reference is given

${ }^{b}$ Browning et al. (2010), ${ }^{c}$ Houdebine (2010), ${ }^{d}$ Reiners et al. (2012), ${ }^{e}$ Stauffer \& Hartmann (1986), ${ }^{f}$ Martínez-Arnáiz et al. (2010), ${ }^{g}$ Reiners \& Basri (2007), ${ }^{h}$ Antonova et al. (2013), ${ }^{j}$ Glebocki \& Gnacinski (2005), ${ }^{k}$ Mohanty \& Basri (2003), ${ }^{l}$ Marcy \& Chen (1992)

Comments:

J03574-011: The resulting temperature corresponds to a late K-dwarf instead of an M2.5-dwarf, as stated by the spectral type. It is very likely that the K-dwarf primary of this star was observed accidentally.

J11026+219: Although Carmencita gives a spectral type of M2.0 for this star, Schöfer (2015) and other literature claim it to be M0.5, which makes the result from the CARMENES template spectrum with a temperature of $3895 \mathrm{~K}$ more reliable than the other results. 
Table A.2.: Measured astrophysical parameters of investigated stars without $v \sin i$ measurement. A default value of $3 \mathrm{kms}^{-1}$ was assumed. The table lists Carmencita identifier (Karmn), spectral type, effective temperature, surface gravity, metallicity, Ca II emission flag, and instrument with which the spectrum was obtained (templ. standing for CARMENES template).

\begin{tabular}{|c|c|c|c|c|c|c|}
\hline Karmn & $\begin{array}{l}\text { Spectral } \\
\text { type }\end{array}$ & $\begin{array}{c}T_{\text {eff }}[\mathrm{K}] \\
( \pm 93)\end{array}$ & $\begin{array}{c}\log g[\mathrm{cgs}] \\
( \pm 0.29)\end{array}$ & $\begin{array}{c}{[\mathrm{Fe} / \mathrm{H}]} \\
( \pm 0.25)\end{array}$ & $\begin{array}{l}\text { Ca II } \\
\text { emission }\end{array}$ & Instr. \\
\hline J00395+149S & $\mathrm{M} 4.0 \mathrm{~V}$ & 3354 & 5.07 & -0.16 & $\ldots$ & HRS \\
\hline J02033-212 & $\mathrm{M} 2.5 \mathrm{~V}$ & 3474 & 4.95 & -0.07 & $\ldots$ & CAFE \\
\hline $\mathrm{J} 03263+171$ & $\mathrm{M} 4.0 \mathrm{~V}$ & 3414 & 5.05 & -0.26 & $\ldots$ & HRS \\
\hline \multirow[t]{2}{*}{$\mathrm{J} 04376+528$} & M0.0 V & 4043 & 4.66 & +0.04 & Yes & CARM. \\
\hline & & 4034 & 4.66 & +0.00 & Yes & templ. \\
\hline $\mathrm{J} 06565+440$ & M4.5 V & 3394 & 5.04 & -0.15 & $\ldots$ & HRS \\
\hline $\mathrm{J} 07353+548$ & $\mathrm{M} 2.0 \mathrm{~V}$ & 3519 & 4.96 & -0.21 & $\ldots$ & CARM. \\
\hline J09011+019 & $\mathrm{M} 3.0 \mathrm{~V}$ & 3514 & 4.95 & -0.16 & $\ldots$ & FEROS \\
\hline $\mathrm{J} 10125+570$ & M3.5 V & 3405 & 5.03 & -0.16 & $\ldots$ & CARM. \\
\hline $\mathrm{J} 11110+304 \mathrm{E}$ & $\ldots$ & 4202 & 4.57 & +0.34 & $\ldots$ & CARM. \\
\hline $\mathrm{J} 11110+304 \mathrm{~W}$ & $\mathrm{M} 2.0 \mathrm{~V}$ & 3550 & 4.97 & -0.29 & $\ldots$ & CARM. \\
\hline J11201-104 & $\mathrm{M} 2.0 \mathrm{~V}$ & 3549 & 5.01 & -0.41 & Yes & CARM. \\
\hline J11306-080 & M3.5 V & 3418 & 5.02 & -0.14 & $\ldots$ & CARM. \\
\hline $\mathrm{J} 12290+417$ & M3.5 V & 3325 & 5.09 & -0.20 & $\ldots$ & CAFE \\
\hline J14307-086 & M0.5 V & 4085 & 4.60 & +0.32 & $\ldots$ & CARM. \\
\hline $\mathrm{J} 16255+260$ & M3.0 V & 3444 & 5.03 & -0.23 & $\ldots$ & CAFE \\
\hline $\mathrm{J} 16487+106$ & $\mathrm{M} 2.5 \mathrm{~V}$ & 3505 & 5.03 & -0.38 & $\ldots$ & FEROS \\
\hline $\mathrm{J} 17033+514$ & M4.5 V & 3225 & 5.09 & +0.04 & $\ldots$ & HRS \\
\hline $\mathrm{J} 18174+483$ & $\mathrm{M} 2.0 \mathrm{~V}$ & 3514 & 4.99 & -0.29 & Yes & CARM. \\
\hline J18480-145 & $\mathrm{M} 2.5 \mathrm{~V}$ & 3496 & 4.96 & -0.18 & $\ldots$ & CARM. \\
\hline J23096-019 & M3.5 V & 3464 & 4.97 & -0.10 & $\ldots$ & FEROS \\
\hline $\mathrm{J} 23113+085$ & M3.5 V & 3380 & 5.03 & -0.08 & $\ldots$ & FEROS \\
\hline
\end{tabular}





\section{Bibliography}

Aceituno, J., Sánchez, S. F., Grupp, F., Lillo, J., Hernán-Obispo, M., Benitez, D., Montoya, L. M., Thiele, U. et AL. (2013) CAFE: Calar Alto Fiber-fed Échelle spectrograph. A\&A, 552, A31.

Allard, F. \& Hauschildt, P. H. (1995) Model atmospheres for M (sub)dwarf stars. 1: The base model grid. ApJ, 445, 433-450.

Allard, F., Hauschildt, P. H., Alexander, D. R., Tamanai, A. \& Schweitzer, A. (2001) The Limiting Effects of Dust in Brown Dwarf Model Atmospheres. ApJ, 556, 357372.

Allard, F., Homeier, D. \& Freytag, B. (2011) Model Atmospheres From Very Low Mass Stars to Brown Dwarfs. In 16th Cambridge Workshop on Cool Stars, Stellar Systems, and the Sun, edited by C. Johns-Krull, M. K. Browning \& A. A. West, vol. 448 of Astronomical Society of the Pacific Conference Series.

Alonso-Floriano, F. J., Morales, J. C., Caballero, J. A., Montes, D., Klutsch, A., Mundt, R., Cortés-Contreras, M., Ribas, I. et al. (2015) CARMENES input catalogue of M dwarfs. I. Low-resolution spectroscopy with CAFOS. A\&A, 577, A128.

Ames, J. S. (1892) The modern spectroscope. The concave grating in theory and practice. Astronomy and Astro-Physics (formerly The Sidereal Messenger), 11, 28-42.

Angelov, T. (1996) Surface gravity along the main sequence. Bulletin Astronomique de Belgrade, 154, 13-16.

Antonova, A., Hallinan, G., Doyle, J. G., Yu, S., Kuznetsov, A., Metodieva, Y., Golden, A. \& Cruz, K. L. (2013) Volume-limited radio survey of ultracool dwarfs. A\&A, 549, A131.

Artigau, É., Kouach, D., Donati, J.-F., Doyon, R., Delfosse, X., Baratchart, S., Lacombe, M., Moutou, C. et al. (2014) SPIRou: the near-infrared spectropolarimeter/high-precision velocimeter for the Canada-France-Hawaii telescope. In Ground-based and Airborne Instrumentation for Astronomy V, vol. 9147 of Proc. SPIE.

Asplund, M., Grevesse, N., Sauval, A. J. \& Scott, P. (2009) The Chemical Composition of the Sun. ARA\&A, 47, 481-522.

Baraffe, I., Chabrier, G., Allard, F. \& Hauschildt, P. H. (1998) Evolutionary models for solar metallicity low-mass stars: mass-magnitude relationships and colormagnitude diagrams. A\&A, 337, 403-412. 
Baraffe, I., Homeier, D., Allard, F. \& Chabrier, G. (2015) New evolutionary models for pre-main sequence and main sequence low-mass stars down to the hydrogenburning limit. A\&A, 577, A42.

Bauer, F. F., Zechmeister, M. \& Reiners, A. (2015) Calibrating echelle spectrographs with Fabry-Pérot etalons. A\&A, 581, A117.

Bettex, A. (1965) The discovery of nature. Simon and Schuster, URL https://books. google.de/books?id=5VNDAAAAIAAJ.

Bond, I. A., Udalski, A., Jaroszyński, M., Rattenbury, N. J., Paczyński, B., Soszyński, I., Wyrzykowski, L., Szymański, M. K. et al. (2004) OGLE 2003-BLG-235/MOA 2003-BLG-53: A Planetary Microlensing Event. ApJ, 606, L155-L158.

Bonfils, X., Delfosse, X., Udry, S., Forveille, T., Mayor, M., Perrier, C., Bouchy, F., Gillon, M. ET AL. (2013) The HARPS search for southern extra-solar planets. XXXI. The M-dwarf sample. A\&A, 549, A109.

Bonfils, X., Delfosse, X., Udry, S., Santos, N. C., Forveille, T. \& Ségransan, D. (2005) Metallicity of M dwarfs. I. A photometric calibration and the impact on the mass-luminosity relation at the bottom of the main sequence. A\&A, 442, 635-642.

Bonfils, X., Mayor, M., Delfosse, X., Forveille, T., Gillon, M., Perrier, C., Udry, S., Bouchy, F. ET AL. (2007) The HARPS search for southern extra-solar planets. X. A m $\sin \mathrm{i}=11 \mathrm{M} \_\oplus$ planetaroundthenearbyspottedMdwarfGJ674.A\&A, 474, 293 -299 .

Boyajian, T. S., von Braun, K., van Belle, G., McAlister, H. A., ten Brummelaar, T. A., Kane, S. R., Muirhead, P. S., Jones, J. et al. (2012) Stellar Diameters and Temperatures. II. Main-sequence K- and M-stars. ApJ, 757, 112.

Boyle, R. (1664) Experiments and Considerations Touching Colours.

Braje, D. A., Kirchner, M. S., Osterman, S., Fortier, T. \& Diddams, S. A. (2008) Astronomical spectrograph calibration with broad-spectrum frequency combs. European Physical Journal D, 48, 57-66.

BretT, J. M. (1995) Opacity sampling model photospheres for M dwarfs. I. Computations, sensitivities and comparisons. A\&A, 295, 736.

Browning, M. K., Basri, G., Marcy, G. W., West, A. A. \& Zhang, J. (2010) Rotation and Magnetic Activity in a Sample of M-Dwarfs. AJ, 139, 504-518.

Buccino, A. P., Lemarchand, G. A. \& Mauas, P. J. D. (2007) UV habitable zones around M stars. Icarus, 192, 582-587.

Caballero, J. A., Cortés-Contreras, M., López-Santiago, J., Alonso-Floriano, F. J., Klutsch, A., Montes, D., Morales, J. C., Mundt, R. et al. (2013) CARMENES. III. CARMENCITA, the input catalogue. In Highlights of Spanish Astrophysics VII, edited by J. C. Guirado, L. M. Lara, V. Quilis \& J. Gorgas. 
Campbell, B., Walker, G. A. H. \& Yang, S. (1988) A search for substellar companions to solar-type stars. ApJ, 331, 902-921.

Charbonneau, D., Irwin, J., Nutzman, P. \& Falco, E. E. (2008) The MEarth Project to Detect Habitable SuperEarth Exoplanets. In American Astronomical Society Meeting Abstracts \#212, vol. 40 of Bulletin of the American Astronomical Society.

Clough, S. A., Shephard, M. W., Mlawer, E. J., Delamere, J. S., Iacono, M. J., CadyPereira, K., Boukabara, S. \& Brown, P. D. (2005) Atmospheric radiative transfer modeling: a summary of the AER codes. J. Quant. Spec. Radiat. Transf., 91, 233244.

Cortes-Contreras, M. (2016) PhD-Thesis, CARMENES-UCM: scientific preparation, multiplicity, chromospheric activity and kinematics. Universidad Complutense de Madrid.

Del Burgo, C., Helling, C., Martín, E. L., Witte, S., Zapatero Osorio, M. R. \& Hauschildt, P. H. (2013) Characterization of 36 late M-dwarfs using spectral energy distributions and near-infrared echelle spectra . Mem. Soc. Astron. Italiana, 84, 1084.

Delfosse, X., Forveille, T., Perrier, C. \& Mayor, M. (1998) Rotation and chromospheric activity in field M dwarfs. A\&A, 331, 581-595.

Delfosse, X., Forveille, T., Ségransan, D., Beuzit, J.-L., Udry, S., Perrier, C. \& Mayor, M. (2000) Accurate masses of very low mass stars. IV. Improved massluminosity relations. A\&A, 364, 217-224.

Dong, S., Gould, A., Udalski, A., Anderson, J., Christie, G. W., Gaudi, B. S., OGLE Collaboration, Jaroszyński, M. et al. (2009) OGLE-2005-BLG-071Lb, the Most Massive M Dwarf Planetary Companion? ApJ, 695, 970-987.

Endl, M., Cochran, W. D., Tull, R. G. \& MacQueen, P. J. (2003) A Dedicated M Dwarf Planet Search Using The Hobby-Eberly Telescope. AJ, 126, 3099-3107.

ESA (2016) ESA Science \& Technology. http://sci.esa.int/gaia/ 47354-fact-sheet/, [Online; accessed 02-March-2016].

Fischer, D. A. \& Valenti, J. (2005) The Planet-Metallicity Correlation. ApJ, 622, 11021117.

Follert, R., Dorn, R. J., Oliva, E., Lizon, J. L., Hatzes, A., Piskunov, N., Reiners, A., Seemann, U. et al. (2014) CRIRES+: a cross-dispersed high-resolution infrared spectrograph for the ESO VLT. In Ground-based and Airborne Instrumentation for Astronomy V, vol. 9147 of Proc. SPIE.

Foo, G., Palacios, D. M. \& Swartzlander, JR., G. A. (2005) Optical vortex coronagraph. Optics Letters, 30, 3308-3310. 
Gaidos, E. \& Mann, A. W. (2014) M Dwarf Metallicities and Giant Planet Occurrence: Ironing Out Uncertainties and Systematics. ApJ, 791, 54.

Ghezzi, L., Cunha, K., Smith, V. V., de Araújo, F. X., Schuler, S. C. \& de la Reza, R. (2010) Stellar Parameters and Metallicities of Stars Hosting Jovian and Neptunian Mass Planets: A Possible Dependence of Planetary Mass on Metallicity. ApJ, 720, 1290-1302.

Gizis, J. E. (1997) M-Subdwarfs: Spectroscopic Classification and the Metallicity Scale. AJ, 113, 806-822.

Glebocki, R. \& Gnacinski, P. (2005) VizieR Online Data Catalog: Catalog of Stellar Rotational Velocities (Glebocki+ 2005). VizieR Online Data Catalog, 3244.

Gonzalez, G. (1997) The stellar metallicity-giant planet connection. MNRAS, 285, 403412.

Gonzalez, G., Laws, C., Tyagi, S. \& Reddy, B. E. (2001) Parent Stars of Extrasolar Planets. VI. Abundance Analyses of 20 New Systems. AJ, 121, 432-452.

Grevesse, N., Asplund, M. \& Sauval, A. J. (2007) The Solar Chemical Composition. Space Sci. Rev., 130, 105-114.

Grimaldi, F. M. (1665) Physico-mathesis de lumine coloribus et iride, libri 2 in quorum 1. afferuntur noua experimenta.

Gustafsson, B., Edvardsson, B., Eriksson, K., Jørgensen, U. G., Nordlund, A. \& Plez, B. (2008) A grid of MARCS model atmospheres for late-type stars. I. Methods and general properties. A\&A, 486, 951-970.

Haberle, R. M., McKay, C. P., Tyler, D. \& Reynolds, R. T. (1996) Can Synchronously Rotating Planets Support An Atmosphere? In Circumstellar Habitable Zones, edited by L. R. Doyle.

Hatzes, A. P. \& Cochran, W. D. (1993) Long-period radial velocity variations in three K giants. ApJ, 413, 339-348.

Hauschildt, P. H., Allard, F. \& Baron, E. (1999) The NextGen Model Atmosphere Grid for $3000 \leq T \_e f f \leq 10,000 K$.ApJ, 512, $377--385$.

Henry, G. W., Marcy, G., Butler, R. P. \& Vogt, S. S. (1999) HD 209458. IAU Circ., 7307.

Herter, T. L., Henderson, C. P., Wilson, J. C., Matthews, K. Y., Rahmer, G., Bonati, M., Muirhead, P. S., Adams, J. D. et al. (2008) The performance of TripleSpec at Palomar. In Ground-based and Airborne Instrumentation for Astronomy II, vol. 7014 of Proc. SPIE. 
Holmberg, J., Nordström, B. \& Andersen, J. (2009) The Geneva-Copenhagen survey of the solar neighbourhood. III. Improved distances, ages, and kinematics. A\&A, 501, 941-947.

Houdebine, E. R. (2010) Observation and modelling of main-sequence star chromospheres - XIV. Rotation of dM1 stars. MNRAS, 407, 1657-1673.

HuANG, S. S. (1959) OCCURRENCE OF LIFE IN THE UNIVERSE. American Scientist, 47(3), 397-402, URL http: //www . jstor . org/stable/27827376.

Husser, T.-O. \& Ulbrich, K. (2014) Using a model for telluric absorption in fullspectrum fits. In Astronomical Society of India Conference Series, vol. 11 of Astronomical Society of India Conference Series.

Husser, T.-O., Wende-von Berg, S., Dreizler, S., Homeier, D., Reiners, A., Barman, T. \& Hauschildt, P. H. (2013) A new extensive library of PHOENIX stellar atmospheres and synthetic spectra. A\&A, 553, A6.

IDA, S. \& Lin, D. N. C. (2004) Toward a Deterministic Model of Planetary Formation. I. A Desert in the Mass and Semimajor Axis Distributions of Extrasolar Planets. ApJ, 604, 388-413.

Irwin, J. M., Berta-Thompson, Z. K., Charbonneau, D., Dittmann, J., Falco, E. E., Newton, E. R. \& Nutzman, P. (2015) The MEarth-North and MEarth-South Transit Surveys: Searching for Habitable Super-Earth Exoplanets Around Nearby M-dwarfs. In 18th Cambridge Workshop on Cool Stars, Stellar Systems, and the Sun, edited by G. T. van Belle \& H. C. Harris, vol. 18 of Cambridge Workshop on Cool Stars, Stellar Systems, and the Sun.

Jeffers, S. V., Schöfer, P., Lamert, A., Reiners, A., Montes, D., Caballero, J., PassegGer, V., Cortes-Contreras, M. eT al. (submitted) CARMENES input catalogue of $\mathrm{M}$ dwarfs, III. Rotation and activity from high-resolution spectroscopic observations. A\&A.

Jones, H. R. A., Longmore, A. J., Allard, F. \& Hauschildt, P. H. (1996) Spectral analysis of M dwarfs. MNRAS, 280, 77-94.

Kaeufl, H.-U., Ballester, P., Biereichel, P., Delabre, B., Donaldson, R., Dorn, R., FEDRIGo, E., FingER, G. ET AL. (2004) CRIRES: a high-resolution infrared spectrograph for ESO's VLT. In Ground-based Instrumentation for Astronomy, edited by A. F. M. Moorwood \& M. Iye, vol. 5492 of Proc. SPIE.

Kalas, P., Fitzgerald, M. P., Clampin, M., Graham, J. R., Chiang, E., Kite, E. S., Stapelfeldt, K. \& Krist, J. (2009) Fomalhaut b: Direct Detection of a $\epsilon$ Jupitermass Object Orbiting Fomalhaut. In American Astronomical Society Meeting Abstracts \#213, vol. 41 of Bulletin of the American Astronomical Society.

Kasting, J. F. (1988) Runaway and moist greenhouse atmospheres and the evolution of earth and Venus. Icarus, 74, 472-494. 
Kasting, J. F., Whitmire, D. P. \& Reynolds, R. T. (1993) Habitable Zones around Main Sequence Stars. Icarus, 101, 108-128.

Kaufer, A., Wolf, B., Andersen, J. \& Pasquini, L. (1997) FEROS, the fiber-fed extended range optical spectrograph for the ESO 1.52-m telescope. The Messenger, 89, 1-4.

Kausch, W., Noll, S., Smette, A., Kimeswenger, S., Barden, M., Szyszka, C., Jones, A. M., SANA, H. ET AL. (2015) Molecfit: A general tool for telluric absorption correction. II. Quantitative evaluation on ESO-VLT/X-Shooterspectra. A\&A, 576, A78.

Kenyon, S. J. \& Hartmann, L. (1995) Pre-Main-Sequence Evolution in the TaurusAuriga Molecular Cloud. ApJS, 101, 117.

Kepler, S. O., Costa, J. E. S., Castanheira, B. G., Winget, D. E., Mullally, F., Nather, R. E., Kilic, M., von Hippel, T. ET AL. (2005) Measuring the Evolution of the Most Stable Optical Clock G 117-B15A. ApJ, 634, 1311-1318.

Kerber, F., Nave, G., Sansonetti, C. J., Bristow, P. \& Rosa, M. R. (2007) The Spectrum of Th-Ar Hollow Cathode Lamps in the 900-4500 nm Region: Establishing Wavelength Standards for the Calibration of VLT Spectrographs. In The Future of Photometric, Spectrophotometric and Polarimetric Standardization, edited by C. Sterken, vol. 364 of Astronomical Society of the Pacific Conference Series.

KIRCHER, A. (1646) Ars magna lucis et umbrae. Rom.

Kornet, K., Bodenheimer, P., Różyczka, M. \& Stepinski, T. F. (2005) Formation of giant planets in disks with different metallicities. A\&A, 430, 1133-1138.

Kotani, T., Tamura, M., Suto, H., Nishikawa, J., Sato, B., Aoki, W., Usuda, T., Kurokawa, T. et aL. (2014) Infrared Doppler instrument (IRD) for the Subaru telescope to search for Earth-like planets around nearby M-dwarfs. In Ground-based and Airborne Instrumentation for Astronomy V, vol. 9147 of Proc. SPIE.

KÜrster, M., Zechmeister, M. \& EndL, M. (2009) The M dwarf planet search program with the VLT+UVES. In 15th Cambridge Workshop on Cool Stars, Stellar Systems, and the Sun, edited by E. Stempels, vol. 1094 of American Institute of Physics Conference Series.

Lamert, A. (2014) Masterthesis, Spectroscopic analysis of Carmenes sample. GeorgAugust Universität Göttingen.

Laws, C., Gonzalez, G., Walker, K. M., Tyagi, S., Dodsworth, J., Snider, K. \& Suntzeff, N. B. (2003) Parent Stars of Extrasolar Planets. VII. New Abundance Analyses of 30 Systems. AJ, 125, 2664-2677.

Lépine, S., Hilton, E. J., Mann, A. W., Wilde, M., Rojas-Ayala, B., Cruz, K. L. \& Gaidos, E. (2013) A Spectroscopic Catalog of the Brightest (J 9) M Dwarfs in the Northern Sky. AJ, 145, 102. 
Lindgren, S., Heiter, U. \& Seifahrt, A. (2016) Metallicity determination of M dwarfs. High-resolution infrared spectroscopy. A\&A, 586, A100.

Lovis, C. \& PePe, F. (2007) A new list of thorium and argon spectral lines in the visible. A\&A, 468, 1115-1121.

Lyot, B. (1939) The study of the solar corona and prominences without eclipses (George Darwin Lecture, 1939). MNRAS, 99, 580.

Mahadevan, S., Ramsey, L. W., Terrien, R., Robertson, P., Marchwinski, R. C., Hearty, F., Levi, E., Kári Stefánsson, G. et al. (2015) The Habitable-zone Planet Finder (HPF): Achieving high precision radial velocities and mitigating stellar activity noise. In American Astronomical Society Meeting Abstracts, vol. 225 of American Astronomical Society Meeting Abstracts.

MaLBET, F. (1996) High angular resolution coronography for adaptive optics. A\&AS, 115, 161.

Maldonado, J., Affer, L., Micela, G., Scandariato, G., Damasso, M., Stelzer, B., BarBIERI, M., BEDIN, L. R. ET AL. (2015) Stellar parameters of early-M dwarfs from ratios of spectral features at optical wavelengths. A\&A, 577, A132.

Mann, A. W., Brewer, J. M., Gaidos, E., Lépine, S. \& Hilton, E. J. (2013a) Prospecting in Late-type Dwarfs: A Calibration of Infrared and Visible Spectroscopic Metallicities of Late K and M Dwarfs Spanning 1.5 dex. AJ, 145, 52.

Mann, A. W., Deacon, N. R., Gaidos, E., Ansdell, M., Brewer, J. M., Liu, M. C., Magnier, E. A. \& Aller, K. M. (2014) Prospecting in Ultracool Dwarfs: Measuring the Metallicities of Mid- and Late-M Dwarfs. AJ, 147, 160.

Mann, A. W., Feiden, G. A., Gaidos, E., Boyajian, T. \& von Braun, K. (2015) How to Constrain Your M Dwarf: Measuring Effective Temperature, Bolometric Luminosity, Mass, and Radius. ApJ, 804, 64.

Mann, A. W., Gaidos, E. \& Ansdell, M. (2013b) Spectro-thermometry of M Dwarfs and Their Candidate Planets: Too Hot, Too Cool, or Just Right? ApJ, 779, 188.

Marci, J. M. (1648) Thaumantias liber de arcu coelesti deque colorum apparentium scaturigine, ab his vero colorigeni riui deriuantur : ducibus, natura, ortu, et causis : in quo pellucidi opticae fontes a sua geometria, et physica hermetoperipatetica.

Marcy, G. W. \& Butler, R. P. (1992) Precision radial velocities with an iodine absorption cell. PASP, 104, 270-277.

Marcy, G. W. \& Chen, G. H. (1992) The rotation of M dwarfs. ApJ, 390, 550-559.

Martín, E. L., Guenther, E., Zapatero Osorio, M. R., Bouy, H. \& Wainscoat, R. (2006) A Multiwavelength Radial Velocity Search for Planets around the Brown Dwarf LP 944-20. ApJ, 644, L75-L78. 
Martínez-Arnáiz, R., Maldonado, J., Montes, D., Eiroa, C. \& Montesinos, B. (2010) Chromospheric activity and rotation of FGK stars in the solar vicinity. An estimation of the radial velocity jitter. A\&A, 520, A79.

Mayor, M., Pepe, F., Queloz, D., Bouchy, F., Rupprecht, G., Lo Curto, G., Avila, G., Benz, W. et Al. (2003) Setting New Standards with HARPS. The Messenger, 114, 20-24.

Mayor, M. \& Queloz, D. (1995) A Jupiter-mass companion to a solar-type star. Nature, 378, 355-359.

Michelson, A. A. (1898) The Echelon Spectroscope. ApJ, 8, 37.

Mohanty, S. \& Basri, G. (2003) Rotation and Activity in Mid-M to L Field Dwarfs. ApJ, 583, 451-472.

Mordasini, C., Alibert, Y., Benz, W. \& Naef, D. (2008) Giant Planet Formation by Core Accretion. In Extreme Solar Systems, edited by D. Fischer, F. A. Rasio, S. E. Thorsett \& A. Wolszczan, vol. 398 of Astronomical Society of the Pacific Conference Series.

Murphy, M. T., Udem, T., Holzwarth, R., Sizmann, A., Pasquini, L., Araujo-Hauck, C., Dekker, H., D'Odorico, S. ET AL. (2007) High-precision wavelength calibration of astronomical spectrographs with laser frequency combs. MNRAS, 380, 839-847.

Neuhäuser, R., Seifahrt, A., Röll, T., Bedalov, A. \& Mugrauer, M. (2007) Detectability of Planets in Wide Binaries by Ground-Based Relative Astrometry with AO. In Binary Stars as Critical Tools Tests in Contemporary Astrophysics, edited by W. I. Hartkopf, P. Harmanec \& E. F. Guinan, vol. 240 of IAU Symposium.

Neves, V., Bonfils, X., Santos, N. C., Delfosse, X., Forveille, T., Allard, F., Natário, C., Fernandes, C. S. et al. (2012) Metallicity of M dwarfs. II. A comparative study of photometric metallicity scales. A\&A, 538, A25.

Neves, V., Bonfils, X., Santos, N. C., Delfosse, X., Forveille, T., Allard, F. \& Udry, S. (2013) Metallicity of M dwarfs. III. Planet-metallicity and planet-stellar mass correlations of the HARPS GTO M dwarf sample. A\&A, 551, A36.

Newton, I. (1672) Philosophical Transactions of the Royal Society of London Series I, 7, 4034-4035.

Newton, S. I. (1730) Opticks, or a treatise of the reflections, refractions, inflections and colours of light. William Innys, URL http://books.google.com/books?id= XXu4AkRVBBoC

Niven, C. (1874) On a method of finding the parallax of double stars and on the displacement of the lines in the spectrum of a planet. MNRAS, 34, 339-347.

Noyes, R. W., Hartmann, L. W., Baliunas, S. L., Duncan, D. K. \& Vaughan, A. H. (1984) Rotation, convection, and magnetic activity in lower main-sequence stars. ApJ, 279, 763-777. 
Nutman, A. P., Bennett, V. C., Friend, C. R. L., Van Kranendonk, M. J. \& R., C. A. (2016) Rapid emergence of life shown by discovery of 3,700-million-year-old microbial structures. , 537, 535-538.

Ochsenbein, F., Bauer, P. \& Marcout, J. (2000) The VizieR database of astronomical catalogues. A\&AS, 143, 23-32.

Orosz, J. A., Welsh, W. F., Carter, J. A., Fabrycky, D. C., Cochran, W. D., Endl, M., Ford, E. B., Haghighipour, N. ET AL. (2012) Kepler-47: A Transiting Circumbinary Multiplanet System. Science, 337, 1511.

Passegger, V. M., Reiners, A., Jeffers, S. V., Wende-von Berg, S., Schöfer, P., Caballero, J., Schweitzer, A., Amado, P. et al. (in prep.) CARMENES input catalogue of M dwarfs, IV. Astrophysical parameters of target stars. A\&A.

Passegger, V. M., Wende-von Berg, S. \& Reiners, A. (2016) Fundamental M-dwarf parameters from high-resolution spectra using PHOENIX ACES models. I. Parameter accuracy and benchmark stars. A\&A, 587, A19.

Pepe, F., Cristiani, S., Rebolo, R., Santos, N. C., Dekker, H., Mégevand, D., Zerbi, F. M., Cabral, A. ET Al. (2013) ESPRESSO An Echelle SPectrograph for Rocky Exoplanets Search and Stable Spectroscopic Observations. The Messenger, 153, 616.

Perryman, M. (2011) The Exoplanet Handbook. Cambridge University Press.

Piskunov, N. E. \& Valenti, J. A. (2002) New algorithms for reducing cross-dispersed echelle spectra. A\&A, 385, 1095-1106.

Pizzolato, N., Maggio, A., Micela, G., Sciortino, S. \& Ventura, P. (2003) The stellar activity-rotation relationship revisited: Dependence of saturated and non-saturated Xray emission regimes on stellar mass for late-type dwarfs. A\&A, 397, 147-157.

Porter, M. J. (2000) Spectroscopy on Small Telescopes: The Echelle Spectrograph. Astrophysics and Space Science, 273, 217-224, URL http://dx . doi .org/10.1023/ A: 1002737017056 .

Quirrenbach, A., Amado, P. J., Mandel, H., Caballero, J. A., Mundt, R., Ribas, I., Reiners, A., Abril, M. ET Al. (2010) CARMENES: Calar Alto high-resolution search for $\mathrm{M}$ dwarfs with exo-earths with a near-infrared Echelle spectrograph. In Groundbased and Airborne Instrumentation for Astronomy III, vol. 7735 of Society of PhotoOptical Instrumentation Engineers (SPIE) Conference Series.

Rajpurohit, A. S., Reylé, C., Allard, F., Homeier, D., Schultheis, M., Bessell, M. S. \& Robin, A. C. (2013) The effective temperature scale of M dwarfs. A\&A, 556, A15.

Rajpurohit, A. S., Reylé, C., Allard, F., Scholz, R.-D., Homeier, D., Schultheis, M. \& BAYo, A. (2014) High-resolution spectroscopic atlas of M subdwarfs. Effective temperature and metallicity. A\&A, 564, A90. 
Redman, S. L., Nave, G. \& Sansonetti, C. J. (2014) The Spectrum of Thorium from 250 $\mathrm{nm}$ to $5500 \mathrm{~nm}$ : Ritz Wavelengths and Optimized Energy Levels. ApJS, 211, 4.

Reiners, A. \& Basri, G. (2007) The First Direct Measurements of Surface Magnetic Fields on Very Low Mass Stars. ApJ, 656, 1121-1135.

Reiners, A. \& Basri, G. (2010) A Volume-Limited Sample of 63 M7-M9.5 Dwarfs. II. Activity, Magnetism, and the Fade of the Rotation-Dominated Dynamo. ApJ, 710, 924-935.

Reiners, A., Joshi, N. \& Goldman, B. (2012) A Catalog of Rotation and Activity in Early-M Stars. AJ, 143, 93.

Reiners, A., Shulyak, D., Anglada-Escudé, G., Jeffers, S. V., Morin, J., ZechmeisTer, M., Kochukhov, O. \& Piskunov, N. (2013) Radial velocity signatures of Zeeman broadening. A\&A, 552, A103.

Rice, E. L., Oppenheimer, R., Zimmerman, N., Roberts, L. C. \& Hinkley, S. (2015) A New Method for Characterizing Very Low-Mass Companions with Low-Resolution Near-Infrared Spectroscopy. PASP, 127, 479-498.

Robertson, P., Mahadevan, S., Endl, M. \& Roy, A. (2014) Stellar activity masquerading as planets in the habitable zone of the M dwarf Gliese 581. Science, 345, 440-444.

Rojas-Ayala, B., Covey, K. R., Muirhead, P. S. \& Lloyd, J. P. (2012) Metallicity and Temperature Indicators in M Dwarf K-band Spectra: Testing New and Updated Calibrations with Observations of 133 Solar Neighborhood M Dwarfs. ApJ, 748, 93.

Rouan, D., Riaud, P., Boccaletti, A., Clénet, Y. \& Labeyrie, A. (2000) The FourQuadrant Phase-Mask Coronagraph. I. Principle. PASP, 112, 1479-1486.

SaAr, S. H., Butler, R. P. \& Marcy, G. W. (1998) Magnetic Activity-related Radial Velocity Variations in Cool Stars: First Results from the Lick Extrasolar Planet Survey. ApJ, 498, L153-L157.

Santos, N. C., Israelian, G. \& Mayor, M. (2004) Spectroscopic [Fe/H] for 98 extra-solar planet-host stars. Exploring the probability of planet formation. A\&A, 415, 11531166.

Schlaufman, K. C. \& Laughlin, G. (2010) A physically-motivated photometric calibration of M dwarf metallicity. A\&A, 519, A105.

Schöfer, P. (2015) Masterthesis, High-resolution Spectroscopy of CARMENCITA Objects. Georg-August Universität Göttingen.

Seager, S. \& Mallén-Ornelas, G. (2003) A Unique Solution of Planet and Star Parameters from an Extrasolar Planet Transit Light Curve. ApJ, 585, 1038-1055.

Ségransan, D., Kervella, P., Forveille, T. \& Queloz, D. (2003) First radius measurements of very low mass stars with the VLTI. A\&A, 397, L5-L8. 
Simms, W. (1840) On the Optical Glass prepared by the late Dr. Ritchie. MmRAS, 11, 165.

Smette, A., Sana, H., Noll, S., Horst, H., Kausch, W., Kimeswenger, S., Barden, M., SzyszKa, C. ET AL. (2015) Molecfit: A general tool for telluric absorption correction. I. Method and application to ESO instruments. A\&A, 576, A77.

Sousa, S. G., Santos, N. C., Mayor, M., Udry, S., Casagrande, L., Israelian, G., Pepe, F., Queloz, D. ET AL. (2008) Spectroscopic parameters for 451 stars in the HARPS GTO planet search program. Stellar $[\mathrm{Fe} / \mathrm{H}]$ and the frequency of exo-Neptunes. A\&A, 487, 373-381.

Stauffer, J. B. \& Hartmann, L. W. (1986) The rotational velocities of low-mass stars. PASP, 98, 1233-1251.

Swartzlander, G., Close, L., Ford, E., Abdul-Malik, R., Kim, J. \& Peters, M. A. (2007) Optical Vortex Coronagraph. In In the Spirit of Bernard Lyot: The Direct Detection of Planets and Circumstellar Disks in the 21st Century.

Tarter, J. C., Backus, P. R., Mancinelli, R. L., Aurnou, J. M., Backman, D. E., Basri, G. S., Boss, A. P., Clarke, A. et al. (2007) A Reappraisal of The Habitability of Planets around M Dwarf Stars. Astrobiology, 7, 30-65.

Tull, R. G., MacQueen, P. J., Good, J., Epps, H. W. \& HET HRS Team (1998) High Resolution Spectrograph for the Hobby-Eberly Telescope. In American Astronomical Society Meeting Abstracts, vol. 30 of Bulletin of the American Astronomical Society.

Udalski, A., Jaroszyński, M., Paczyński, B., Kubiak, M., Szymański, M. K., Soszyński, I., Pietrzyński, G., Ulaczyk, K. et al. (2005) A Jovian-Mass Planet in Microlensing Event OGLE-2005-BLG-071. ApJ, 628, L109-L112.

Valenti, J. A. \& Fischer, D. A. (2005) Spectroscopic Properties of Cool Stars (SPOCS). I. 1040 F, G, and K Dwarfs from Keck, Lick, and AAT Planet Search Programs. ApJS, 159, 141-166.

Valenti, J. A. \& Piskunov, N. (1996) Spectroscopy made easy: A new tool for fitting observations with synthetic spectra. A\&AS, 118, 595-603.

Valenti, J. A., Piskunov, N. \& Johns-Krull, C. M. (1998) Spectral Synthesis of TiO Lines. ApJ, 498, 851.

Van Belle, G. T. \& von Braun, K. (2009) Directly Determined Linear Radii and Effective Temperatures of Exoplanet Host Stars. ApJ, 694, 1085-1098.

van Straten, W., Bailes, M., Britton, M., Kulkarni, S. R., Anderson, S. B., Manchester, R. N. \& Sarkissian, J. (2001) A test of general relativity from the threedimensional orbital geometry of a binary pulsar. Nature, 412, 158-160. 
Wadsworth, F. L. O. (1895) The Modern Spectroscope. XI. Some New Designs of Combined Grating and Prismatic Spectroscopes of the Fixed-Arm Type, and a New Form of Objective Prism. ApJ, 1, 232.

Wadsworth, F. L. O. (1898) Notes on the Use of the Grating in Stellar Spectroscopic Work. ApJ, 7, 198.

Waterhouse, J. (1890) Spectrum Photography with Rowland's Concave Diffraction Gratings. Memorie della Societa Degli Spettroscopisti Italiani, 18, 14.

Wilken, T., Lovis, C., Manescau, A., Steinmetz, T., Pasquini, L., Lo Curto, G., Hänsch, T. W., Holzwarth, R. et al. (2010) High-precision calibration of spectrographs. MNRAS, 405, L16-L20.

Wolszczan, A. \& Frail, D. A. (1992) A planetary system around the millisecond pulsar PSR1257 + 12. Nature, 355, 145-147.

Wuchterl, G., Guillot, T. \& Lissauer, J. J. (2000) Giant Planet Formation. Protostars and Planets $I V, 1081$.

Zboril, M. \& Byrne, P. B. (1998) Metallicity and photospheric abundances in field K and M dwarfs. MNRAS, 299, 753-758.

Zechmeister, M., Anglada-Escudé, G. \& Reiners, A. (2014) Flat-relative optimal extraction. A quick and efficient algorithm for stabilised spectrographs. A\&A, 561, A59. 


\section{Acknowledgements}

I would like to thank my supervisor Ansgar for his guidance and support during my $\mathrm{PhD}$. His advice and experience is always helpful and improved my work considerably. Working in his group is very inspiring and led to many useful discussions and interesting collaborations.

Thanks to Sebastian for helping me at the beginning of my $\mathrm{PhD}$ and introducing me to the topic.

Thanks to Sandra, Andreas, Bárbara and José, who helped to improve my understanding of the field. I very much appreciated their support and the fruitful discussions with them. A great thank you to all my friends, not only in Göttingen, but all over the world. In this context, I would like to especially thank Sudeshna, Chris, Luis, Laura, Eli, Magdalena, Lena and Miriam, who always have an open ear and helped me with advice when needed in difficult scientific and private situations throughout the last years.

I also thank my office mates, Elisabeth, Vaishali, Marius and Patrick. The occasional distractions and non-scientific chats are a nice disturbance at any time.

Finally, I would like to thank my mother and brother for all their support on my way. I know that I can always feel welcome and understood at home. 